\title{
As Propriedades P e RP em espaços de Banach
}

\author{
Neusa Nogas Tocha
}

\author{
Dissertação Apresentada ao \\ INSTITUTO DE MATEMÁTICA E ESTATÍSTICA \\ da \\ UNIVERSIDADE DE SÃO PAULO \\ para a obtenção do grau de mestre em matemática \\ Área de concentração: Análise \\ Orientadora: Profa. Dra. Mary Lilian Lourenço
} apoio financeiro da CAPES

São Paulo, fevereiro de 2002 


\section{As Propriedades P e RP \\ em \\ espaços de Banach}

Este exemplar corresponde à redação final

da dissertação devidamente corrigida e

defendida por Neusa Nogas Tocha

e aprovada pela comissão julgadora.

São Paulo, 22 de fevereiro de 2002.

Banca examinadora:

- Profa. Dra. Mary Lilian Lourenço (orientadora) - IME-USP

- Prof. Dr. Geraldo Botelho - UFU

- Prof. Dr. Raymundo Alencar - IMECC-UNICAMP 
Dedico este trabalho aos meus pais Mário e Nádia e ao meu esposo Maurício 


\section{Agradecimentos}

Agradeço à minha orientadora Profa. Dra. Mary Lilian pela confiança em mim depositada, pelo incentivo e sugestões sem os quais este trabalho dificilmente seria realizado. Agradeço pela sua orientação, paciência e companheirismo durante estes anos.

Agradeço a hospitalidade e companheirismo dos professores e colegas que, diretamente ou indiretamente, contribuiram para a realização deste trabalho.

Agradeço à minha família, principalmente meus pais, pelo apoio constante aos meus estudos.

Agradeço ao meu esposo Maurício pelo seu apoio, companheirismo, sua tolerância e sua eterna disposição em me ajudar.

Finalmente, agradeço a CAPES pelo apoio financeiro recebido durante o programa de mestrado.

( $)$ Obrigado $(\star)$ 


\title{
Resumo
}

O objetivo deste trabalho é estudar os espaços de Banach que possuem as propriedades denominadas $\mathbf{P}$ e RP. Estas propriedades foram introduzidas por Aron, Choi \& Llavona em [2].

Vamos estudar a propriedade $\mathbf{P}$ e a propriedade $\mathbf{R P}$ e suas relações com outras propriedades de espaços de Banach, entre elas, a propriedade Dunford-Pettis, polinomialmente Schur e Schur.

\begin{abstract}
The main purpose of this work is to study Banach spaces with properties $\mathbf{P}$ and $\mathbf{R P}$. These properties were introduced by Aron, Choi \& Llavona, in [2].

We study properties $(\mathbf{P})$ and $(\mathbf{R P})$ and their relation with other properties of Banach spaces, such as the Dunford-Pettis, the polynomially Schur and Schur property.
\end{abstract}




\section{Sumário}

Introdução

1 Preliminares $\quad 7$

1.1 Notação . . . . . . . . . . . . . . . . . . . . . . . . . . . 7

1.2 Definições e resultados . . . . . . . . . . . . . . . . . . 8

1.3 Alguns resultados da geometria de Espaços de Banach . . . . . . . . . . . 19

2 Polinômios 23

2.1 Aplicações multilineares . . . . . . . . . . . . . . . . . . . . . . 23

2.2 Fórmula multinomial . . . . . . . . . . . . . . . . . . . . . . 29

2.3 Polinômios homogêneos . . . . . . . . . . . . . . . . . 35

2.4 Polinômios contínuos . . . . . . . . . . . . . . . . . . . 40

2.5 Polinômios de tipo finito, fracamente contínuos e compactos . . . . . . . . 50

3 Propriedade $\mathbf{P} \quad 59$

3.1 Introdução . . . . . . . . . . . . . . . . . . . . . . . . . . . . . . . . 59

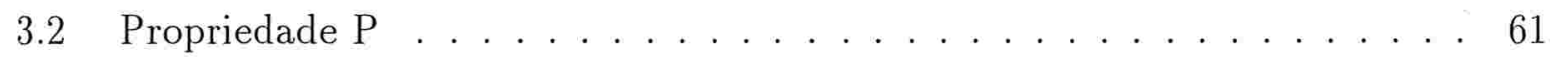

4 Recíproca da propriedade $\mathrm{P} \quad 81$

4.1 Propriedade RP . . . . . . . . . . . . . . . . . . . 81

4.2 Funções polinomialmente contínuas sobre os limitados . . . . . . . . . . . 84

4.3 Exemplos de alguns espaços que não possuem a propriedade RP . . . . . 91

$\begin{array}{ll}\text { Referências Bibliográficas } & 101\end{array}$ 


\section{Introdução}

O estudo do espaço dos polinômios homogêneos contínuos definidos em espaços de Banach tem sido objeto contínuo de pesquisa nos últimos anos. Um dos pontos a se destacar é o grande interesse em estabelecer relações entre um espaço de Banach $X$ e o espaço dos polinômios definidos sobre $X$. Podemos observar tal fato nos diversos trabalhos científicos que estudam como os polinômios portam-se em conexão com certas propriedades geométricas dos espaços de Banach onde estão definidos e de propriedades definidas em termos de polinômios. Citamos aqui alguns trabalhos [2], [8], [9], [12], [16], [18], [19], [20] e [23] que abordam o referido tema.

Nesta dissertação temos por objetivo estudar as propriedades $\mathbf{P}$ e $\mathbf{R P}$, introduzidas por Aron, Choi \& Llavona em [2], e suas relações com as propriedades do espaço de Banach em questão. Os resultados apresentados neste trabalho se encontram em [2], [8], [9] e [12].

A motivação original para a definição da propriedade $\mathbf{P}$ é dada pelo seguinte problema:

Sejam $X, Y$ espaços de Banach sobre o corpo $\mathbb{K}$ onde $B_{X}$ e $B_{Y}$ são as bolas unitárias fechadas de $X$ e $Y$ respectivamente, e $g: B_{X} \longrightarrow B_{Y}$ uma função que satisfaz a seguinte propriedade $(\star)$ :

( ) Para qualquer polinômio contínuo $P: Y \longrightarrow \mathbb{K}, P \circ g: B_{X} \longrightarrow \mathbb{K}$ é uniformemente contínua.

Seria g necessariamente uniformemente continua?

Aron, Choi \& Llavona no artigo [2] observaram que o problema tem resposta positiva se a seguinte condição é satisfeita:

$(\star \star)$ : Se para quaisquer duas sequências limitadas $\left(u_{n}\right)_{n},\left(v_{n}\right)_{n}$ em $Y$, se para cada $P \in \mathcal{P}(Y) \operatorname{com} \mid P\left(u_{n}\right)-P\left(v_{n}\right) \stackrel{n \rightarrow \infty}{\longrightarrow} 0$ implicar que $\left\|u_{n}-v_{n}\right\| \stackrel{n \rightarrow \infty}{\longrightarrow} 0$.

Para poder determinar sob que condições os espaços de Banach tem a propriedade 
$(\star \star)$, Aron, Choi \& Llavona em [2] introduziram uma propriedade mais fraca que $(\star \star)$, e a chamaram de propriedade $\mathbf{P}$. Eles observaram que se uma função $g: B_{X} \longrightarrow B_{Y}$ verifica $(\star)$ e $Y$ é um espaço polinomialmente Schur e tem a propriedade $\mathbf{P}$ então $g$ é uniformemente contínua.

No capítulo 1 apresentamos as notações, definições, resultados e algumas propriedades geométricas de espaços de Banach que utilizamos no decorrer da dissertação.

No capítulo 2 estudamos as aplicações multilineares, a Fórmula de Leibniz, os polinômios homogêneos e as condições necessárias e suficientes para que um polinômio seja contínuo. Indicamos [11] e [27] como livros textos para o estudo desses tópicos. Também introduzimos um breve estudo sobre polinômios de tipo finito, fracamente contínuos e compactos.

No capítulo 3 temos por objetivo estudar a propriedade $\mathbf{P}$ e suas relações com outras propriedades de espaços de Banach, entre elas, a Dunford-Pettis e polinomialmente Schur. Para exibirmos um número maior de exemplos de espaços que possuem a propriedade $\mathbf{P}$, também estudamos as suas relações com as propriedades de espaços de tipo p, $S_{p}$ e $W_{p}$.

Aron, Choi \& Llavona em [2] colocaram a seguinte pergunta:

Existe um espaço de Banach que não tem a propriedade $\mathbf{P}$ ?

Em [12], Choi \& Kim conseguiram encontrar condições suficientes para que um espaço não tenha a propriedade $\mathbf{P}$. Tais condições foram estudados neste capítulo.

No trabalho [9], Castillo, García \& Gonzallo conseguiram exibir um exemplo de um espaço de Banach que não possui a propriedade $\mathbf{P}$. Tal espaço é dado por um produto cartesiano de espaços que têm a propriedade $\mathbf{P}$. O exemplo trabalha com o espaço de sequências de Lorentz e seu predual. Finalizamos o capítulo 3 estudando este exemplo.

No capítulo 4 estudamos a recíproca da propriedade $\mathbf{P}$, a qual é denominada de propriedade RP. Assim como no capítulo 3, aqui estudamos as suas relações com as propriedades de espaços de Banach citadas anteriormente. Também estudamos alguns exemplos de espaços de Banach que não satisfazem a propriedade RP. 


\section{Capítulo 1}

\section{Preliminares}

Neste capítulo apresentamos as notações e resultados necessários que serão utilizados no decorrer desta dissertação e cujas demonstrações podem ser encontradas nas respectivas referências indicadas.

\subsection{Notação}

As propriedades topológicas envolvidas no decorrer da dissertação estarão associadas à topologia dada pela norma em questão. A menos que se mencione algo, vamos utilizar a seguinte notação.
$\mathbb{N}$
o conjunto dos números naturais
$\mathbb{R}$
o corpo dos números reais
$\mathbb{C}$
o corpo dos números complexos
$\mathbb{K}$
o corpo $\mathbb{R}$ ou $\mathbb{C}$
$X, Y$
espaços normados sobre $\mathbb{K}$
$X^{m}$
o espaço $X \times \stackrel{m}{m} \times X$
$B_{X}(a ; r)$
a bola aberta de $X$ de centro $a$ e raio $r$
$B_{X}[a ; r]$
a bola fechada de $X$ de centro $a$ e raio $r$
$B_{X}$
a bola unitária fechada de $X$
$\mathcal{L}(X ; Y)$
o conjunto da aplicações lineares e contínuas de $X$ em $Y$
$\mathcal{L}\left(X_{1}, \ldots, X_{m} ; Y\right)$
o conjunto das aplicações $m$-lineares contínuas de $X_{1} \times \ldots \times X_{m}$ em $Y$ 


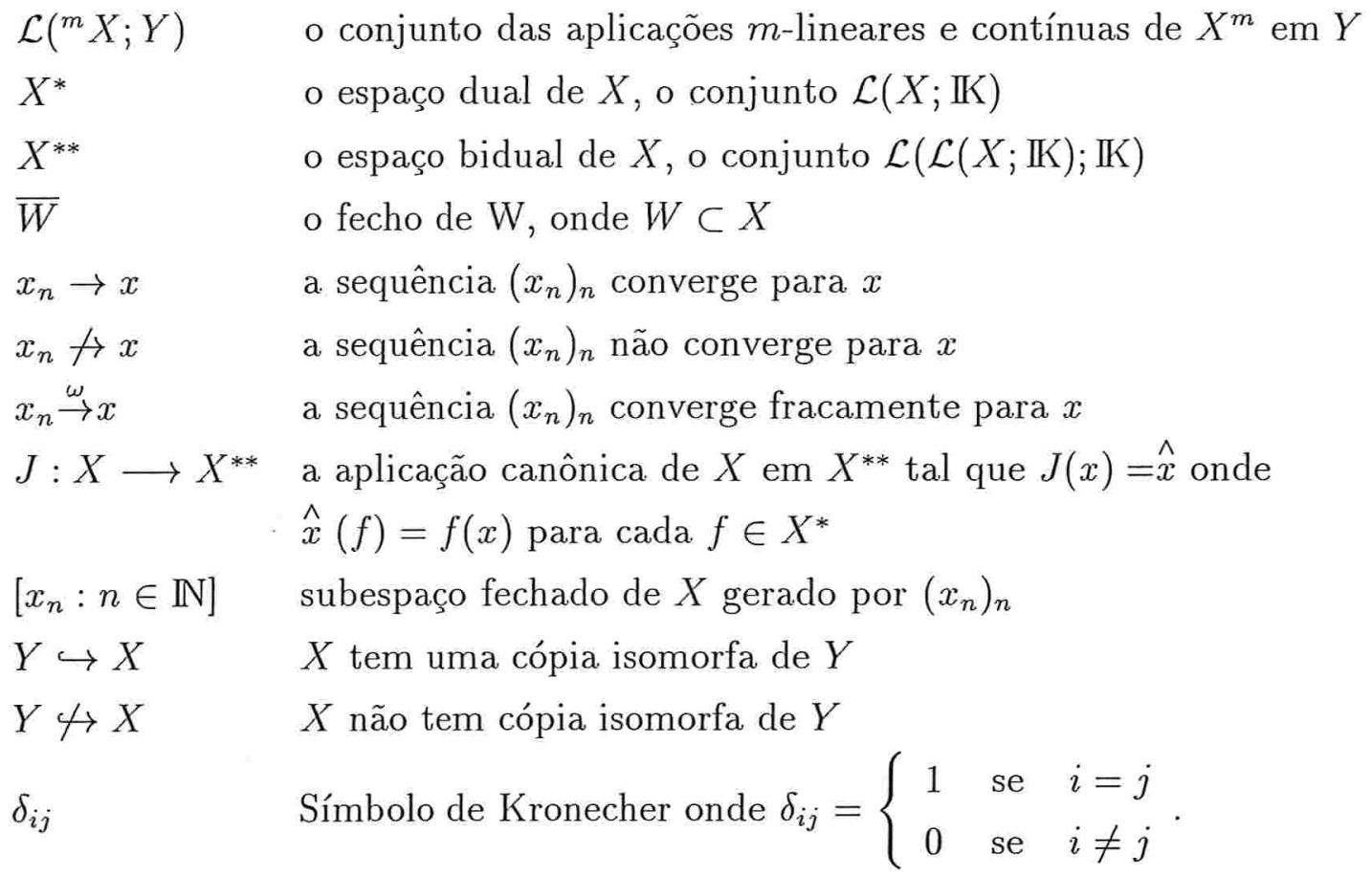

\subsection{Definições e resultados}

Definição 1.2.1. Seja $X$ um espaço normado. Dizemos que $X$ é separável se admite um subconjunto enumerável denso.

O próximo teorema (Banach-Mazur) será utilizado no capítulo 3, onde vamos estudar a denominada propriedade $\mathrm{P}$.

Teorema 1.2.2. (Banach-Mazur)

Seja X um espaço de Banach. Se Xé separável então existe um aplicação linear contínua e sobrejetora $T: l_{1} \longrightarrow X$.

Demonstração: Ver por exemplo [7], teorema 1 pg 114.

Definição 1.2.3. Sejam $X, Y$ espaços normados sobre $\mathbb{I K}$ e $A: X \longrightarrow Y$ uma aplicação linear. Dizemos que $A$ é limitada se existe $M>0$ tal que $\|A(x)\| \leq M\|x\|$ para qualquer $x \in X$.

Teorema 1.2.4. Sejam $X, Y$ espaços normados e $A: X \longrightarrow Y$ uma aplicação linear, as seguintes condições são equivalentes: 
1. A é contínua.

2. A é contínua na origem.

3. Existe $M$ tal que $\|A(x)\| \leq M\|x\|$ para todo $x \in X$, ou seja, A é limitada.

4. A imagem de $A$ aplicada em conjuntos limitados são conjuntos limitados.

Demonstração: Ver por exemplo [11], teorema 2.3.

Teorema 1.2.5. Sejam $X$ um espaço normado e $Y$ um espaço de Banach. A aplicação $\|\cdot\|: \mathcal{L}(X ; Y) \longrightarrow \mathbb{R}$ definida para cada $A \in \mathcal{L}(X ; Y)$ por:

$$
\|A\|=\sup _{x \in B_{X}}\|A(x)\|
$$

constitui uma norma em $\mathcal{L}(X ; Y)$ que o faz completo, ou seja, $\mathcal{L}(X ; Y)$ é um espaço de Banach.

Demonstração: Ver por exemplo [11], teorema 2.5.

O teorema de Hahn-Banach será utilizado no capítulo 3 para demonstrar que um espaço de Banach com certas propriedades geométricas, tem uma determinada propriedade polinomial tratada em tal capítulo.

Teorema 1.2.6. (Hahn-Banach)

Sejam $X$ um espaço normado, $Y$ um subespaço próprio de $X$ e $f \in Y^{*}$. Então existe $\phi \in X^{*}$ tal que:

$$
\left\{\begin{array}{rl}
\phi(z) & =f(z) \quad \text { para cada } z \in Y \\
& e \\
\|\phi\| & =\|f\|
\end{array} .\right.
$$

Demonstração: Ver por exemplo [24], teorema 4.3-2 pg 221.

Definições 1.2.7. 1. Sejam $X, Y$ espaços normados sobre $\mathbb{K}$. Dizemos que $X$ e $Y$ são isomorfos, e denotamos por $X \cong Y$, se existe uma aplicação linear contínua bijetora $A: X \longrightarrow Y$ com inversa contínua.

2. Um isomorfismo isométrico entre $X$ e $Y$ é um isomorfismo $A: X \longrightarrow Y$ entre $X e$ $Y$ tal que $\|A(x)\|=\|x\|$ para qualquer $x \in X$ e denotamos por $X \approx Y$ quando $X$ e $Y$ são isometricamente isomorfos. 
Proposição 1.2.8. Sejam $X$ um espaço de Banach, $J: X \longrightarrow X^{* *}$ definida por $J(x)=\hat{x}$ para cada $x \in X$, onde para cada $x \in X, \hat{x}(f)=f(x)$ para todo $f \in X^{*}$. Então a aplicação $J$ é uma isometria linear e é chamada de aplicação canônica.

Demonstração: Ver por exemplo [24], lemas 4.6-1 e 4.6-2 pg 240.

Definição 1.2.9. Seja $X$ um espaço de Banach. Dizemos que $X$ é reflexivo se $J$ é sobrejetora.

\section{Teorema 1.2.10. (Teorema de Banach-Steinhaus)}

Sejam $X$ um espaço de Banach, $Y$ um espaço normado e $\left(A_{n}\right)_{n}$ uma sequência em $\mathcal{L}(X ; Y)$. Se para cada $x \in X$ a sequência $\left(A_{n}(x)\right)_{n}$ converge para $A(x)$, então $A \in \mathcal{L}(X ; Y)$.

Demonstração: Ver por exemplo [11], pag 100.

Definição 1.2.11. Sejam $X, Y$ espaços de Banach. Dizemos que $X$ possui cópia isomorfa de $Y$, e denotamos por $Y \hookrightarrow X$, se existir $A \in \mathcal{L}(X ; Y)$ injetora tal que $A^{-1}: A(Y) \longrightarrow Y$ é contínua.

Definições 1.2.12. Seja $X$ um espaço de Banach e $\left(x_{n}\right)_{n}$ uma sequência em $X$.

1. Dizemos que $\left(x_{n}\right)_{n}$ é uma base de Schauder para $X$ se para cada $x \in X$ existe uma única sequência $\left(a_{n}\right)_{n}$ em $\mathbb{K K}$ tal que $\lim _{n \rightarrow \infty}\left\|x-\sum_{i=1}^{n} a_{i} x_{i}\right\|=0$.

2. Dizemos que $\left(x_{n}\right)_{n}$ é uma sequência básica em $X$ se $\left(x_{n}\right)_{n}$ é base de Schauder para o subespaço fechado $\left[x_{n}: n \in \mathbb{N}\right]$.

Neste trabalho, uma sequência $\left(x_{n}\right)_{n} \subset X$ será chamada de base quando ela for uma base de Schauder para $X$.

Definição 1.2.13. Seja $X$ um espaço de Banach. Um par de sequências $\left(x_{n}, x_{n}^{*}\right), x_{n} \in X$, $x_{n}^{*} \in X^{*}$, é chamado de sistema biortogonal para $X$ se $x_{n}^{*}\left(x_{m}\right)=\delta_{n m}, n, m=1,2, \ldots$. 
Seja $X$ um espaço de Banach com base $\left(x_{n}\right)_{n}$. Para cada $n \in \mathbb{N}$ definimos o coeficiente funcional $x_{n}^{*}$ associado ao vetor $x_{n}$ por $x_{n}^{*}\left(\sum_{i=1}^{\infty} a_{i} x_{i}\right)=a_{n}$ para cada $x=\sum_{i=1}^{\infty} a_{i} x_{i}$. Assim temos:

Teorema 1.2.14. Sejam X um espaço de Banach e $\left(x_{n}\right)_{n}$ uma base para $X$ com coeficientes funcionais associados $\left(x_{n}^{*}\right)_{n}$. Então os coeficientes funcionais são contínuos e existe $M>0$ tal que $1 \leq\left\|x_{n}\right\| \cdot\left\|x_{n}^{*}\right\| \leq M$ para todo $n \in \mathbb{N}$. Consequentemente

- $\inf _{n}\left\|x_{n}\right\|>0$ se e somente se $\sup _{n}\left\|x_{n}^{*}\right\|<\infty$.

- $\sup _{n}\left\|x_{n}\right\|<\infty$ se e somente se $\inf _{n}\left\|x_{n}^{*}\right\|>0$.

Demonstração: Ver por exemplo [25], pg 07.

Desta maneira, se $\left(x_{n}\right)_{n}$ é uma base para $X$ e $\left(x_{n}^{*}\right)_{n}$ os coeficientes funcionais associados, então o par $\left(x_{n}, x_{n}^{*}\right)$ é um sistema biortogonal para $X$.

Seja $\left(\Omega, \sum, \mu\right)$ um espaço de medida. Se uma propriedade vale exceto em um conjunto de medida nula, dizemos que vale quase sempre e abreviamos por q.s..

Definições 1.2.15. (os espaços $L_{p}^{\prime} s$ )

Dado qualquer $p, 1 \leq p<\infty$, considere $L_{p}([a, b])$ como sendo o conjunto formado pelas classes de equivalências $\left[f: \int_{a}^{b}|f(t)|^{p} d t<\infty\right]$ das funções mensuráveis de $[a, b] \mathrm{em}$ IK. Uma função $g \in\left[f: \int_{a}^{b}|f(t)|^{p} d t<\infty\right]$ se $f$ e $g$ coincidem quase sempre.

As operações usuais de adição e multiplicação por escalar fazem de $L_{p}([a, b])$ um espaço vetorial e a aplicação definida no espaço a valores em $\mathbb{R}$ dada por

$$
\|f\|_{p}=\left(\int_{a}^{b}|f(t)|^{p} d t\right)^{1 / p}
$$

constitui uma norma (a usual) em $L_{p}([a, b])$ que o faz completo, ou seja, $\left(L_{p}([a, b]),\|\cdot\|_{p}\right)$ é um espaço de Banach.

Para $p=\infty, L_{\infty}([a, b])$ é o conjunto formado pelas classes das funções mensuráveis $[f:$ ess sup $|f(t)|<\infty]$, onde ess $\sup |f(t)|=\inf \{M:|f(t)| \leq M$ q.s. $\}$.

$A$ aplicação definida no espaço a valores em $\mathbb{R}$ dada por

$$
\|f\|_{\infty}=\operatorname{ess} \sup |f(t)|
$$


o faz completo, ou seja, $\left(L_{\infty}([a, b]),\|\cdot\|_{\infty}\right)$ é um espaço de Banach.

Para $1 \leq p \leq \infty$, se $1 \leq p<q$, então $L_{q}([a, b]) \subset L_{p}([a, b])$ e $\|\cdot\|_{p} \leq\|\cdot\|_{q}$.

Definição 1.2.16. (o espaço $C[a, b]$ )

Dado um intervalo fechado $[a, b]$ de $\mathbb{R}, a \neq b$, considere $C[a, b]$ o conjunto formado pelas funções continuas $f$ de $[a, b] \mathrm{em} \mathbb{K}$.

As operações usuais de adição e multiplicação por escalar fazem de $C[a, b]$ um espaço vetorial e a aplicação definida no espaço a valores em $\mathbb{R}$ dada por

$$
\|f\|=\sup |f(t)|
$$

o faz completo, ou seja, $(C[a, b],\|\cdot\|)$ é um espaço de Banach.

Definições 1.2.17. ( os espaços $l_{p} s$ e $c_{0}$ )

Dado qualquer $p, 1 \leq p<\infty$, considere $l_{p}$ como sendo o conjunto das sequências $x=\left(x_{k}\right)_{k} \subset \mathbb{K}$ tais que a série $\sum_{k=1}^{\infty}\left|x_{k}\right|^{p}$ converge.

As operações usuais de adição e multiplicação por escalar fazem de $l_{p}$ um espaço vetorial e a aplicação no espaço a valores em $\mathbb{R}$ dada por

$$
\|x\|_{p}=\left(\sum_{k=1}^{\infty}\left|x_{k}\right|^{p}\right)^{1 / p}
$$

constitui uma norma em $l_{p}$ (a usual) que o faz completo, ou seja, $\left(l_{p},\|\cdot\|\right)$ é um espaço de Banach.

Para $p=\infty$, considere $l_{\infty}$ como sendo o conjunto das sequências $x=\left(x_{k}\right)_{k} \subset \mathbb{I K}$ limitadas. As operaçôes usuais de adição e multiplicação por escalar fazem de $l_{\infty}$ um espaço vetorial e a aplicação definida no espaço a valores em $\mathbb{R}$ dada por

$$
\|x\|_{\infty}=\sup _{k \in \mathbb{N}}\left|x_{k}\right|
$$

constitui uma norma em $l_{\infty}$ que o faz completo, ou seja, $\left(l_{\infty},\|\cdot\|\right)$ é um espaço de Banach.

Definimos $c_{0}$ como sendo o subespaço de $l_{\infty}$ constituído pelas sequências $x=\left(x_{i}\right)_{i} \subset \mathbb{K}$ que convergem a zero.

Munindo $c_{0}$ da norma induzida de $l_{\infty}$, o espaço normado obtido é Banach. 
Para $1 \leq p \leq \infty$, se $1 \leq p<q$, então $l_{p} \subset l_{q}$ e $\|\cdot\|_{q} \leq\|\cdot\|_{p}$.

\section{Definição 1.2.18. (operador linear compacto)}

Sejam $X, Y$ espaços normados e $A: X \longrightarrow Y$ um operador linear. Dizemos que $A$ é compacto se $A$ aplica cada subconjunto $M \subset X$ limitado, em um subconjunto $A(M)$ relativamente compacto em $Y$.

Alguns exemplos de operadores compactos:

a) Para $1 \leq p \leq \infty$, o operador $T: l_{p} \longrightarrow l_{p}$ definido por $T(x)=\left(\frac{x_{j}}{j}\right)_{j}$ para cada $x=\left(x_{j}\right)_{j} \in l_{p}$ é compacto.

b) Seja $X$ um espaço normado com produto interno. Sejam $y, z \in X$ fixos e $T: X \longrightarrow X$ dado por $T(x)=\langle x, y\rangle z$ é um operador compacto.

c) Sejam $X$ um espaço normado, $z \in X$ fixo e $f \in X^{*}$. O operador $T: X \longrightarrow X$ definido por $T(x)=f(x) z$ é compacto.

Teorema 1.2.19. Sejam $X, Y$ espaços normados e $A: X \rightarrow Y$ um operador linear. $A$ é compacto se e somente se $A$ aplica sequências limitadas $\left(x_{n}\right)_{n} \subset X$ em sequências $\left(A\left(x_{n}\right)\right)_{n} \subset Y$ que admitem subsequências convergentes.

Demonstração: Ver por exemplo [24], teorema 8.1-3 pg 407.

Definição 1.2.20. Seja $X$ um espaço vetorial normado. A topologia fraca de $X$ é a topologia obtida tomando como base todos os conjuntos da forma:

$$
U\left(x_{0}, x_{1}^{*}, \ldots, x_{n}^{*}, \epsilon\right)=\left\{x \in X: \sup _{i=1, \ldots, n}\left|x_{i}^{*}(x)-x_{i}^{*}\left(x_{0}\right)\right|<\epsilon\right\}
$$

onde $x_{0} \in X ; x_{1}^{*}, \ldots, x_{n}^{*} \in X$ e $\epsilon>0$.

Aqui, fracamente aberto, fracamente fechado, fracamente compacto, função fracamente contínua, aplicação fracamente compacta, etc estão se referindo a conjunto aberto, fechado, compacto, função contínua, aplicação compacta, etc com relação à topologia fraca, e denotamos por $\bar{W}^{\omega}$ o fecho fraco do conjunto $W$. 
Teorema 1.2.21. Seja $X$ um espaço de Banach. Então $X$ é reflexivo se e somente se $B_{X}$ é fracamente compacta.

Demonstração: Ver por exemplo [13], pg 18.

Proposição 1.2.22. Sejam $X, Y$ espaços de Banach e $\left(x_{n}\right)_{n}$ uma sequência em $X$.

1. Se $x_{n} \rightarrow x$ para algum $x \in X$ então $\left(x_{n}\right)_{n}$ converge fracamente para $x$, e denotamos por $x_{n} \stackrel{\omega}{\rightarrow} x$, ou seja, para cada $f \in X^{*}, f\left(x_{n}\right) \rightarrow f(x)$.

2. Se $x_{n} \stackrel{\omega}{\rightarrow} x$ para algum $x \in X$ então $A\left(x_{n}\right) \stackrel{\omega}{\rightarrow} A(x)$ para cada $A \in \mathcal{L}(X ; Y)$.

Demonstração: Ver por exemplo [24], teorema 4.8-4.

Proposição 1.2.23. Sejam $X, Y$ espaços de Banach e $A \in \mathcal{L}(X ; Y)$. Se $A$ é compacta então $A$ é completamente contínua, ou seja, para cada sequência $\left(x_{n}\right)_{n}$ em $X$ tal que $x_{n} \stackrel{\omega}{\longrightarrow} x$ para algum $x$ em $X$ temos que $A\left(x_{n}\right) \rightarrow A(x)$.

Demonstração: Ver por exemplo [24], teorema 8.1-7 pg 410.

\section{Teorema 1.2.24. (Eberlein - Smulian)}

Sejam X um espaço de Banach, e W um subconjunto de X. Então as seguintes afirmações são equivalentes:

1. W é fracamente sequencialmente compacto, ou seja, qualquer sequência de $W$ tem uma subsequência que converge fracamente para algum elemento de $X$;

2. O fecho fraco de $W$ é fracamente compacto, isto é, $W$ é relativamente fracamente compacto.

Demonstração: Ver por exemplo, [13], pg 18.

Definição 1.2.25. Sejam $X, Y$ espaços de Banach. Uma aplicação linear $A: X \rightarrow Y$ é denominada fracamente compacta se, para cada $W \subset X$ limitado, temos que $A(W)$ é relativamente fracamente compacto em $Y$.

Os seguintes resultados da teoria de medida serão necessários no capítulo 4: 
Teorema 1.2.26. Seja $f$ uma função limitada e Lebesgue mensurárel em $[a, b]$. Então para cada $\epsilon>0$ existe uma função escada $h$ tal que $\int_{a}^{b}|f(t)-h(t)| d t<\epsilon$.

Demonstração: Ver por exemplo [6], pg 72.

Teorema 1.2.27. Seja $\left(\Omega, \sum, \mu\right)$ um espaço de medida. Uma sequência $\left(f_{n}\right)_{n} \mathrm{em}$ $L_{1}\left(\Omega, \sum, \mu\right)$ converge fracamente para $f \in L_{1}\left(\Omega, \sum, \mu\right)$ se e somente se $\left(f_{n}\right)_{n}$ é limitada e

$$
\int_{E} f(t) d \mu=\lim _{n \rightarrow \infty} \int_{E} f_{n}(t) d \mu
$$

para cada $E \in \sum$.

Demonstração: Ver por exemplo [13], pg 91.

Definição 1.2.28. Sejam $X, Y$ espaços métricos. Uma função $f: X \longrightarrow Y$ é uniformemente contínua sobre $X$ se dado $\epsilon>0$ existe $\delta>0$ tal que $\|f(x)-f(y)\|<\epsilon$ sempre que $\|x-y\|<\delta$.

Teorema 1.2.29. Sejam $X, Y$ espaços métricos. Então $f: X \longrightarrow Y$ é uniformemente contínua se e somente se para quaisquer duas sequências $\left(x_{n}\right)_{n},\left(y_{n}\right)_{n}$ em X com $\left\|x_{n}-y_{n}\right\| \stackrel{n \rightarrow \infty}{\longrightarrow} 0$ temos que $\left\|f\left(x_{n}\right)-f\left(y_{n}\right)\right\| \stackrel{n \rightarrow \infty}{\longrightarrow} 0$.

Demonstração: $(\Rightarrow)$ Seja $f: X \longrightarrow Y$ uniformemente contínua, então dado $\epsilon>0$ existe $\delta>0$ tal que $\|f(x)-f(y)\|<\epsilon$ sempre que $\|x-y\|<\delta$. Se $\left(x_{n}\right)_{n},\left(y_{n}\right)_{n}$ são duas sequências em $X$ tais que $\left\|x_{n}-y_{n}\right\| \stackrel{n \rightarrow \infty}{\longrightarrow} 0$, então para o $\delta$ encontrado acima existe $n_{0} \in \mathbb{N}$ tal que $\left\|x_{n}-y_{n}\right\|<\delta$ para todo $n \geq n_{0}$, logo $\left\|f\left(x_{n}\right)-f\left(y_{n}\right)\right\|<\epsilon$ para todo $n \geq n_{0}$. Portanto $\left\|f\left(x_{n}\right)-f\left(y_{n}\right)\right\| \stackrel{n \rightarrow \infty}{\longrightarrow} 0$.

$(\Leftarrow)$ Suponhamos que $f$ não é uniformemente contínua, então existe $\epsilon>0$ com a seguinte propriedade: para cada $n \in \mathbb{N}$ encontramos pontos $x_{n}, y_{n}$ tal que $\left\|x_{n}-y_{n}\right\|<\frac{1}{n}$ e $\left\|f\left(x_{n}\right)-f\left(y_{n}\right)\right\| \geq \epsilon$. Então teríamos duas sequências $\left(x_{n}\right)_{n},\left(y_{n}\right)_{n}$ tal que $\left\|x_{n}-y_{n}\right\| \stackrel{n \rightarrow \infty}{\longrightarrow} 0$, mas $\left\|f\left(x_{n}\right)-f\left(y_{n}\right)\right\| \geq \epsilon$, e isto contradiz a hipótese.

Portanto $f$ é uniformemente contínua.

Dizemos que uma aplicação $A: X \longrightarrow Y$ é fracamente uniformemente contínua sobre os limitados se para cada $W \subset X$ limitado e cada $\epsilon>0$ existem $\phi_{1}, \ldots, \phi_{n} \in X^{*}$ e $\delta>0$ tais que $\|A(x)-A(y)\|<\epsilon$ sempre que $\left|\phi_{i}(x-y)\right|<\delta \operatorname{com} x, y \in W$ e $i=1, \ldots, n$. 
No que segue vamos apresentar um teorema que será utilizado no capítulo 2 , seção 2.5. A demonstração do teorema encontra-se em [[5], proposição 2.5].

Lema 1.2.30. Sejam $X, Y$ espaços de Banach e $T: X \longrightarrow Y$ uma aplicação linear uniformemente contínua sobre os limitados de $X$. Então $T(W)$ é precompacto para todo $W \subset X$ limitado.

Demonstração: Seja $\epsilon>0$. Para cada $x \in W$, seja $V(x, \epsilon):=\left\{y \in Y ;\|y-T(x)\|_{Y}<\epsilon\right\}$. Como $T$ é fracamente uniformemente contínua sobre $W$, existem $\phi_{1}, \ldots, \phi_{n} \in X^{*}$ e $\delta>0$ tais que $x, z \in W$ e $\left|\phi_{i}(x-z)\right|<\delta$, $(1 \leq i \leq n)$, temos que $\|T(x)-T(z)\|_{Y}<\epsilon$. Consideremos a aplicação:

$$
\begin{aligned}
\Phi: X \longrightarrow\left(\mathbb{R}^{n},\|\cdot\|\right) \quad \text { onde }\|x\|=\sup _{1 \leq i \leq n}\left|x_{i}\right| \\
x \longmapsto\left(\phi_{1}(x), \ldots, \phi_{n}(x)\right)
\end{aligned}
$$

Para cada $x_{0} \in X$ arbitrário, temos que $\left\|\Phi(x)-\Phi\left(x_{0}\right)\right\|=\left\|\left(\phi_{1}\left(x-x_{0}\right), \ldots, \phi_{n}\left(x-x_{0}\right)\right)\right\|=\sup _{1 \leq i \leq n}\left|\phi_{i}\left(x-x_{0}\right)\right|$. Como para todo $1 \leq i \leq n,\left|\phi_{i}\left(x-x_{0}\right)\right| \leq\left\|\phi_{i}\right\| \cdot\left\|x-x_{0}\right\| x$, tomando $M=\max _{1 \leq i \leq n}\left\|\phi_{i}\right\|$ segue que $\left\|\Phi(x)-\Phi\left(x_{0}\right)\right\| \leq M\left\|x-x_{0}\right\|_{X}$. Donde se conclui que $\Phi$ é limitada e contínua. Logo $\Phi(W)$ é um subconjunto limitado de $\mathbb{R}^{n}$. Então existe $c>0$ tal que $\Phi(W) \subset[-c, c] \times \stackrel{n}{n} \times[-c, c]$ que é compacto. Logo $\Phi(W)$ é precompacto. Seja $U=B(0, \delta)=\left\{x \in \mathbb{R}^{n} ;\|x\|<\delta\right\}$. Como $\Phi(W)$ é precompacto, existem $x_{1}, \ldots, x_{m} \in W$ tais que $\Phi(W)=\bigcup_{i=1}^{m} \Phi\left(x_{i}\right)+U$. Dado $x \in W$ existe algum $x_{j}, 1 \leq j \leq m$, tal que $\left\|\Phi(x)-\Phi\left(x_{j}\right)\right\|<\delta$, isto é, $\sup _{1 \leq i \leq n}\left|\phi_{i}\left(x-x_{j}\right)\right|<\delta$. Logo $\left|\phi_{i}(x)-\phi_{i}\left(x_{j}\right)\right|<\delta$ para todo $1 \leq i \leq n$ o que implica que $\left\|T(x)-T\left(x_{j}\right)\right\|_{Y}<\epsilon$. Então $T(W) \subset V\left(x_{1}, \epsilon\right) \bigcup \ldots \bigcup V\left(x_{m}, \epsilon\right)$, isto é, $T(W)$ é precompacto.

Teorema 1.2.31. Sejam $X, Y$ espaços de Banach sobre o corpo IK e $A: X \rightarrow Y$ uma aplicação linear contínua. Então $A$ é compacta se e somente se $A$ é fracamente uniformemente contínua sobre os subconjuntos limitados de $X$.

Demonstração: $(\Rightarrow)$ Seja $B=\left\{x \in X ;\|x\|_{X} \leq 1\right\}$. Suponhamos que $A$ é compacta; então $\overline{A(B)}$ é um subconjunto compacto de $Y$. Seja $\mathrm{K}=\overline{A(B)}+\overline{A(B)}$; K é um subconjunto compacto de $Y$, pois por Tychonoff $\overline{A(B)} \times \overline{A(B)}$ é compacto em $Y \times Y$ e como a operação adição é contínua, leva compactos em compactos. Pelo teorema de [[24], 
teorema 4.3-3 pg 223] para cada $k \in \mathrm{K}$ existe $\phi_{k} \in Y^{*}$ tal que $\left\|\phi_{k}\right\|=1$ e $\left|\phi_{k}(k)\right|=\|k\|_{Y}$. Seja $\phi: Y \longrightarrow \mathbb{R}$ definida por $\phi(x)=\|x\|_{K}-\left|\phi_{k}(x)\right|, \phi$ é contínua, pois é diferença de funções contínuas. Logo $\left.\phi\right|_{K}$ é contínua, isto é, dado $\epsilon>0$ existe uma vizinhança $U_{k}$ de $k$ em $Y$ tal que se $x \in U_{k}$ então $|\phi(x)-\phi(k)|<\epsilon$. Segue daí que $\|x\|_{Y}<\epsilon+\left|\phi_{k}(x)\right|$ para todo $x \in U_{k}(\star)$. Como $\mathrm{K} \subset \bigcup_{k \in K} U_{k}$, existem $k_{1}, \ldots, k_{n} \in K$ tais

que $\mathrm{K} \subset \bigcup_{i=1}^{n} U_{k_{i}}$, pois $\mathrm{K}$ é compacto. Assim, se $k \in \mathrm{K}, k \in U_{k_{i}}$ para algum $i \in\{1, \ldots, n\}$ por $(\star)$ temos que

$$
\|k\|_{Y}<\epsilon+\left|\phi_{k_{i}}(k)\right|<\sup _{1 \leq i \leq n}\left|\phi_{k_{i}}(k)\right|+\epsilon
$$

Para cada $i \in\{1, \ldots, n\}$ consideremos o funcional $\phi_{k} \circ A: X \longrightarrow \mathbb{R}$. Sejam $x, y \in B$ tais que $\left|\phi_{k_{i}}(A(x)-A(y))\right|<\epsilon, 1 \leq i \leq n$. Ora, $A(x)-A(y) \in \mathrm{K}$; então por (1.1) temos que:

$$
\|A(x)-A(y)\|_{Y} \leq \sup _{1 \leq i \leq n}\left|\phi_{k_{i}}(A(x)-A(y))\right|+\epsilon<2 \epsilon .
$$

Donde se conclui que $A$ é fracamente uniformemente contínua sobre os subconjuntos limitados de $X$.

$(\Leftarrow)$ Pelo lema acima temos que $A\left(B_{X}\right)$ é precompacto, logo $\overline{A\left(B_{X}\right)}$ é fechado e precompacto o que equivale a dizer que $\overline{A\left(B_{X}\right)}$ é compacto.

Donde se conclui que $A$ é compacta.

\section{Teorema 1.2.32. (Bessaga-Pelczynski)}

Sejam X um espaço de Banach e $\left(x_{n}\right)_{n}$ uma sequência em $X$. Se $x_{n} \stackrel{\omega}{\rightarrow} 0$ e $\left\|x_{n}\right\|=1$ então $\left(x_{n}\right)_{n}$ admite uma subsequência $\left(x_{n_{k}}\right)_{k}$ tal que $\left(x_{n_{k}}\right)_{k}$ é uma sequência básica em $X$.

Demonstração: Ver por exemplo [13], pg 42.

\section{Teorema 1.2.33. (Rosenthal)}

Seja X um espaço de Banach. Toda sequência limitada $\left(x_{n}\right)_{n} \subset X$ admite uma subsequência $\left(x_{n_{k}}\right)_{k}$ fracamente de Cauchy em $X$ se e somente se $l_{1} \nrightarrow X$.

Demonstração: Ver por exemplo [13], cap. XI.

Definição 1.2.34. (O espaço das sequências de Lorentz)

Considere dados $p, 1 \leq p<\infty$, e $w=\left(w_{n}\right)_{n}$ uma sequência decrescente de reais positivos tais que $w_{1}=1$, $\lim _{n \rightarrow \infty} w_{n}=0$ e $\sum_{n=1}^{\infty} w_{n}=\infty$, ou seja, $w \in c_{0} / l_{1}$. A partir destes dados, 
podemos definir o denominado espaço de sequências de Lorentz $d(w ; p)$, isto é, o espaço formado pelas sequências de números reais $x=\left(x_{n}\right)_{n}$ tais que

$$
\sup _{\pi \in \sigma}\left(\sum_{n=1}^{\infty}\left|x_{\pi(n)}\right|^{p} \cdot w_{n}\right)^{1 / p}<\infty
$$

onde $\sigma$ é o conjunto das permutações em $\mathbb{N}$. O espaço d(w;p) munido das operações usuais de adição e multiplicação por real é um espaço vetorial. Quando munimos $d(w ; p)$ com a norma

$$
\|x\|_{p}=\sup _{\pi \in \sigma}\left(\sum_{n=1}^{\infty}\left|x_{\pi(n)}\right|^{p} \cdot w_{n}\right)^{1 / p}
$$

$\left(d(w ; p),\|\cdot\|_{p}\right)$ é um espaço de Banach.

O espaço de sequências de Lorentz $d(w ; p)$ é reflexivo se e somente se $p>1$ (ver por exemplo [25]).

Aqui, neste trabalho, especificamente no capítulo 3, vamos estudar este tipo de espaço, mas no caso $p=1$.

O espaço $d(w ; 1)$ admite um predual (ver por exemplo [30]), que denotamos por $d_{*}(w ; 1)$, que é definido por:

$$
d_{*}(w ; 1)=\left\{x=\left(x_{n}\right)_{n} \in c_{0}: \lim _{k \rightarrow \infty} \frac{\sum_{n=1}^{k} \hat{x}_{n}}{\sum_{n=1}^{k} w_{n}}=0\right\}
$$

onde $\left(\hat{x}_{n}\right)_{n}$ é um rearranjamento decrescente de $\left(\left|x_{n}\right|\right)_{n}$.

Este espaço munido da norma $\|\cdot\|: d_{*}(w ; 1) \longrightarrow \mathbb{R}$ dada por:

$$
\|x\|=\sup _{k \in \mathbb{N}} \frac{\sum_{n=1}^{k} \hat{x}_{n}}{\sum_{n=1}^{k} w_{n}}
$$

o faz completo, ou seja, $\left(d_{*}(w ; p),\|\cdot\|\right)$ é um espaço de Banach.

No capítulo 4, as funções de Rademacher têm um papel fundamental para o estudo dos resultados que garantem que os espaços $l_{\infty}, L_{\infty}[0,1], C[0,1], L_{1}[0,1]$ e $L_{1}[0,2 \pi]$ não possuem a denominada propriedade RP definida no referido capítulo. 
Definição 1.2.35. (Funções de Rademacher)

Para cada $n=0,1,2, \ldots$, , as funções de Rademacher, $r_{n}:[0,1] \longrightarrow \mathbb{R}$, são definidas por:

$$
\begin{gathered}
r_{0}(t)=1 \text { para todo } \quad t \in[0,1] \\
r_{n}(t)=\operatorname{sign}\left(\operatorname{sen}\left(2^{n} \pi t\right)\right), \quad n=1,2, \ldots
\end{gathered}
$$

Proposição 1.2.36. Em $L_{2}[0,1]$, a sequência $\left(r_{n}\right)_{n=0}^{\infty}$ das funções de Rademacher formam um sistema ortonormal, mas não formam uma base ortonormal.

Demonstração: Ver por exemplo [15], pg 10.

\subsection{Alguns resultados da geometria de Espaços de Banach}

Aqui nesta seção vamos apresentar algumas definições e principais resultados da geometria de espaços de Banach que serão utilizados nos capítulos 3 e 4 .

Definições 1.3.1. Sejam $X$ um espaço de Banach sobre o corpo $\mathbb{K}$ e $1 \leq p<\infty$.

1. Dizemos que uma sequência $\left(x_{n}\right)_{n} \subset X$ é fracamente $p$-somável se para cada $x^{*} \in X^{*}$ temos que $\left(x^{*}\left(x_{n}\right)\right)_{n} \in l_{p}$.

2. Dizemos que $X$ tem a propriedade $S_{p}$ se toda sequência $\left(x_{n}\right)_{n} \subset X$ com $x_{n} \stackrel{\omega}{\longrightarrow} 0$ admite uma subsequência $\left(x_{n_{j}}\right)_{j}$ fracamente q-somável, onde $\frac{1}{p}+\frac{1}{q}=1$.

3. Dizemos que $X$ tem a propriedade $W_{p}$ se para cada sequência $\left(x_{n}\right)_{n} \subset X$ limitada existem uma subsequência $\left(x_{n_{k}}\right)_{k}$ de $\left(x_{n}\right)_{n}$ e $x \in X$ tais que $\left(x_{n_{k}}-x\right)_{k}$ é fracamente p-somável.

Facilmente vemos que $W_{1} \subset W_{2} \subset \ldots \equiv\{$ espaços reflexivos $\}$.

Em espaços reflexivos a propriedade $W_{p}$ é equivalente a propriedade $S_{q}$, onde $\frac{1}{p}+\frac{1}{q}=1$ (ver por exemplo [10]). 
Exemplos 1.3.2. (Espaços de Banach com propriedade $S_{p}$ )

1. Para $1<p<\infty, L_{p}[0,1]$ tem a propriedade $S_{r}$ onde $r=\min \{\mathscr{2}, p\}$.

2. Para $1<p<\infty$, $l_{p}$ tem a propriedade $S_{p}$.

3. O espaço $l_{1}$ tem propriedade $S_{p}$ para todo $1 \leq p \leq \infty$.

Definições 1.3.3. Seja X um espaço de Banach.

1. Seja $Y$ um espaço de Banach. Dizemos que $Y$ é finitamente representável em $X$ se para cada $\epsilon>0$ e cada $Y_{0} \subset Y$, $\operatorname{dim} Y_{0}<\infty$, existem $X_{0} \subset X$ com $\operatorname{dim} X_{0}<\infty e$ $T: Y_{0} \longrightarrow X_{0}$ isomorfismo tal que $\|T\| \cdot\left\|T^{-1}\right\|<1+\epsilon$.

2. Dizemos que $X$ é super-reflexivo se para cada $Y$ finitamente representável em $X, Y$ é reflexivo.

Segue da definição que os espaços super-reflexivos também são reflexivos.

Teorema 1.3.4. Seja X um espaço de Banach.

1. X é super-reflexivo se e somente se $X^{*}$ é super-reflexivo.

2. Se $X$ é de dimensão infinita e é super-reflexivo então $X$ tem a propriedade $W_{p}$ para algum $p>1$ ( ou $X \in S_{q}$, pois $S_{q}$ e $W_{p}$ são equivalentes em espaços reflexivos).

Demonstração: Para (1) ver por exemplo [22], teorema 2 pg 897 e para (2) ver por exemplo [10], teorema 2.2 pg 258.

Definição 1.3.5. Seja $X$ um espaço de Banach.

Para cada $\epsilon>0$ seja $\delta(\epsilon)=\inf \left\{1-\left\|\frac{x+y}{2}\right\|:\|x\|=\|y\|=1,\|x-y\| \geq \epsilon\right\}$.

Dizemos que $X$ é uniformemente convexo se $\delta(\epsilon)>0$ para todo $\epsilon>0$, e chamamos $\delta(\epsilon)$ de módulo de convexidade de $X$.

Teorema 1.3.6. Sejam X, Y espaços de Banach.

1. Se $X$ é uniformemente convexo e $Y$ finitamente representável em $X$, então $Y$ é uniformemente convexo. 
2. Se $X$ é uniformemente convexo então $X$ é reflexivo.

Demonstração: Ver por exemplo [7].

Exemplos 1.3.7. Para $p, 1<p<\infty$, os espaços $l_{p}$ e $L_{p}[0,1]$ são uniformementes convexos.

Corolário 1.3.8. Seja X um espaço de Banach. Se $X$ é uniformemente convexo então $X$ é super-reflexivo.

Demonstração: Seja $Y$ finitamente representável em $X$ então pelo teorema 1.3.6 temos que $Y$ é reflexivo. Assim, por definição, $X$ é super-reflexivo.

Exemplos 1.3.9. Os espaços $l_{p}$ e $L_{p}[0,1]$, para $1<p<\infty$, são super-reflexivos.

Definições 1.3.10. Seja X um espaço de Banach.

1. Dizemos que $X$ é de tipo $p, 1 \leq p \leq 2$, (respectivamente, de cotipo $q, 2 \leq q \leq \infty$ ) se existe uma constante $C>0$ tal que para qualquer família finita $\left\{x_{1}, \ldots, x_{n}\right\} \subset X$, temos:

$$
\frac{1}{2^{n}} \sum_{\epsilon_{i}= \pm 1}\left\|\sum_{i=1}^{n} \epsilon_{i} x_{i}\right\| \leq C\left(\sum_{i=1}^{n}\left\|x_{i}\right\|^{p}\right)^{1 / p}
$$

respectivamente,

$$
\left(\sum_{i=1}^{n}\left\|x_{i}\right\|^{q}\right)^{1 / q} \leq C \frac{1}{2^{n}} \sum_{\epsilon_{i} \pm 1}\left\|\sum_{i=1}^{n} \epsilon_{i} x_{i}\right\|
$$

2. Dizemos que $X$ tem tipo não-trivial se $X$ tem tipo $p$ para algum $p>1$.

Observamos que se $X$ é um espaço de tipo não-trivial então $X^{*}$ também é de tipo não-trivial (ver por exemplo [15], pag 263 e 266).

Exemplos 1.3.11. (Relação de tipo e cotipo em alguns espaços de Banach)

1. $l_{1}$ não tem tipo não-trivial, tem cotipo 2 .

2. Se $1 \leq p \leq 2$ então $l_{p}$ tem tipo $p$ e cotipo 2 . 
3. Se $2 \leq q<\infty$ então $l_{q}$ tem tipo 2 e cotipo $q$.

4. Para $1 \leq p<\infty$, o espaço $L_{p}[0,1]$ tem tipo $\min \{p, 2\}$ e cotipo $\max \{p, 2\}$.

Teorema 1.3.12. Seja $X$ um espaço de Banach.

1. Se $X$ tem tipo $p \in(1,2]$ então $X \in S_{r}$ para todo $r<p$.

Demonstração: Ver por exemplo [19], corolário 1.5 pg 219.

Definição 1.3.13. Seja $X$ um espaço de Banach. Dizemos que $X$ é Schur se para cada sequência $\left(x_{n}\right)_{n} \subset X$ com $x_{n} \stackrel{\omega}{\rightarrow} x$ para algum $x \in X$ temos que $x_{n} \stackrel{n \rightarrow \infty}{\longrightarrow} x$.

Exemplos 1.3.14. 1. $l_{1}$ é Schur.

2. $l_{p}, 1<p<\infty$ não é Schur.

3. c co não é Schur.

4. Se $X$ é Schur e se $Y \subset X$ é um subespaço fechado então $Y$ é Schur. 


\section{Capítulo 2}

\section{Polinômios}

\subsection{Aplicações multilineares}

Neste capítulo apresentamos um estudo sobre as aplicações multilineares e polinômios. Aqui o nosso objetivo é estudar as aplicações multilineares contínuas, simétricas, os polinômios m-homogêneos, polinômios m-homogêneos contínuos, os polinômios contínuos e seus principais resultados que nos são necessários para o desenvolvimento da dissertação. Os resultados aqui apresentados encontram-se em [11] e [27].

As aplicações multilineares nos são de grande valia para a definição de polinômios e no estudo dos principais resultados.

Definição 2.1.1. Sejam $m \in \mathbb{N}, X_{1}, \ldots, X_{m}, Y$ espaços vetoriais sobre o corpo $\mathbb{K}$. Dizemos que uma aplicação $A: X_{1} \times \ldots \times X_{m} \longrightarrow Y$ é m-linear (multilinear) se é linear em cada variável, ou seja,

$A\left(x_{1}, \ldots, x_{i}+\lambda \cdot y_{i}, x_{i+1}, \ldots, x_{m}\right)=A\left(x_{1}, \ldots, x_{i}, \ldots, x_{m}\right)+\lambda \cdot A\left(x_{1}, \ldots, x_{i-1}, y_{i}, x_{i+1}, \ldots, x_{m}\right)$ para cada $i=1, \ldots, m$.

Se $X_{1}, \ldots, X_{m}$ são espaços normados, o espaço $X_{1} \times \cdots \times X_{m}$ também é normado munido da norma

$$
\left\|\left(x_{1}, \ldots, x_{m}\right)\right\|=\max _{1 \leq i \leq m}\left\|x_{i}\right\| .
$$

Vamos denotar por $\mathcal{L}_{a}\left(X_{1}, \ldots, X_{m} ; Y\right)$ o espaço vetorial de todas as aplicações $\mathrm{m}$ lineares A : $X_{1} \times \ldots \times X_{m} \longrightarrow Y$. Quando $X=X_{1}=\ldots=X_{m}$ denotamos por 
$\mathcal{L}_{a}\left({ }^{m} X ; Y\right)$. Se $m=0, \mathcal{L}_{a}\left({ }^{m} X ; Y\right)=$ Y. Se $m=1, \mathcal{L}_{a}\left({ }^{m} X ; Y\right)=\mathcal{L}_{a}(X ; Y)$. Quando $Y=\mathbb{K}, \mathcal{L}_{a}\left(X_{1}, \ldots, X_{m} ; Y\right)=\mathcal{L}_{a}\left(X_{1}, \ldots, X_{m}\right)$.

O teorema que apresentamos a seguir nos dá alguns critérios de continuidade para as aplicações multilineares.

Teorema 2.1.2. Sejam $X_{1}, \ldots, X_{m}, Y$ espaços normados sobre o corpo $\mathbb{K}$, $A: X_{1} \times \ldots \times X_{m} \longrightarrow Y$ uma aplicação m-linear. São equivalentes:

\section{A é contínua.}

2. A é contínua na origem.

3. Existe $M>0$ tal que para cada $\left(x_{1}, \ldots, x_{m}\right) \in X_{1} \times \ldots \times X_{m}$ temos que

$$
\left\|A\left(x_{1}, \ldots, x_{m}\right)\right\| \leq M\left\|x_{1}\right\| \ldots\left\|x_{m}\right\|
$$

Demonstração: $(1 \Rightarrow 2)$ Trivial.

$(2 \Rightarrow 3)$ Sejam $A \in \mathcal{L}_{a}\left(X_{1}, \ldots, X_{m} ; Y\right)$ e $x=\left(x_{1}, \ldots, x_{m}\right) \in X_{1} \times \ldots \times X_{m}$. Se $x_{i}=0$ para algum $i=1, \ldots, m$, vale (3) para qualquer $M>0$. Como $A$ é contínua na origem, dado $\epsilon=1$ existe $r>0$ tal que para qualquer $x \in X_{1} \times \ldots \times X_{m}$, com $\|x\| \leq r$, temos que $\|A(x)\| \leq 1$. Seja $x=\left(x_{1}, \ldots, x_{m}\right) \in X_{1} \times \ldots \times X_{m}$ tal que $x_{i} \neq 0$ para todo $i=1, \ldots, m$. Agora para cada $i=1, \ldots, m$, chamamos $z_{i}=\frac{r x_{i}}{\left\|x_{i}\right\|}$ e $z=\left(z_{1}, \ldots, z_{m}\right)$, assim $\|z\|=r$ e

$$
\left\|A\left(z_{1}, \ldots, z_{m}\right)\right\|=\frac{r^{m}}{\left\|x_{1}\right\| \ldots\left\|x_{m}\right\|}\left\|A\left(x_{1}, \ldots, x_{m}\right)\right\| \leq 1
$$

e consequentemente,

$$
\left\|A\left(x_{1}, \ldots, x_{m}\right)\right\| \leq \frac{1}{r^{m}}\left\|x_{1}\right\| \ldots\left\|x_{m}\right\| .
$$

Logo para termos (3), basta considerar $M=\frac{1}{r^{m}}$.

$(3 \Rightarrow 1)$ Sejam $a=\left(a_{1}, \ldots, a_{m}\right) \in X_{1} \times \ldots \times X_{m}$. Então para qualquer $x=\left(x_{1}, \ldots, x_{m}\right) \in X_{1} \times \ldots \times X_{m}$, temos que

$$
\begin{aligned}
A(x)-A(a)= & A\left(x_{1}-a_{1}, x_{2}, \ldots, x_{m}\right)+A\left(a_{1}, x_{2}-a_{2}, x_{3}, \ldots, x_{m}\right)+ \\
& \ldots+A\left(a_{1}, \ldots, a_{m-1}, x_{m}-a_{m}\right) .
\end{aligned}
$$


Assim

$$
\begin{aligned}
\|A(x)-A(a)\| \leq & \left\|A\left(x_{1}-a_{1}, x_{2}, \ldots, x_{m}\right)\right\|+\left\|A\left(a_{1}, x_{2}-a_{2}, x_{3}, \ldots, x_{m}\right)\right\|+ \\
& \ldots+\left\|A\left(a_{1}, \ldots, a_{m-1}, x_{m}-a_{m}\right)\right\| \\
\leq & M\left(\left\|x_{1}-a_{1}\right\|\left\|x_{2}\right\| \ldots\left\|x_{m}\right\|\right. \\
& +\left\|a_{1}\right\|\left\|x_{2}-a_{2}\right\|\left\|x_{3}\right\| \ldots\left\|x_{m}\right\|+ \\
& \left.\ldots+\left\|a_{1}\right\| \ldots\left\|a_{m-1}\right\|\left\|x_{m}-a_{m}\right\|\right) .
\end{aligned}
$$

Se $\|x-a\| \leq 1$ então $\left\|x_{i}\right\| \leq\left\|a_{i}\right\|+1 \leq\|a\|+1$ para cada $i=1, \ldots, m$. Logo

$$
\begin{aligned}
\|A(x)-A(a)\| \leq & M\left(\left\|x_{1}-a_{1}\right\|(\|a\|+1)^{m-1}+\left\|x_{2}-a_{2}\right\|(\|a\|+1)^{m-1}+\right. \\
& \left.\ldots+\left\|x_{m}-a_{m}\right\|(\|a\|+1)^{m-1}\right) \\
= & M(\|a\|+1)^{m-1}\left(\left\|x_{1}-a_{1}\right\|+\ldots+\left\|x_{m}-a_{m}\right\|\right) \\
= & M(\|a\|+1)^{m-1} m\|x-a\| .
\end{aligned}
$$

Portanto dado $\epsilon>0$ existe $\delta=\min \left\{1, \frac{\epsilon}{m M(\|a\|+1)^{m-1}}\right\}$ tal que se $\|x-a\|<\delta$, então $\|A(x)-A(a)\|<\epsilon$.

Vamos denotar por $\mathcal{L}\left(X_{1}, \ldots, X_{m} ; Y\right)$ o espaço vetorial de todas as aplicações $\mathrm{m}$ lineares contínuas A : $X_{1} \times \ldots \times X_{m} \rightarrow Y$. Quando $X=X_{1}=\ldots=X_{m}$ denotamos por $\mathcal{L}\left({ }^{m} X ; Y\right)$. Se $m=0$, denotamos $\mathcal{L}\left({ }^{m} X ; Y\right)=$ Y. Se $m=1$, denotamos $\mathcal{L}\left({ }^{m} X ; Y\right)=\mathcal{L}(X ; Y)$. Quando $Y=\mathbb{K}$, denotamos $\mathcal{L}\left(X_{1}, \ldots, X_{m} ; Y\right)=\mathcal{L}\left(X_{1}, \ldots, X_{m}\right)$.

Para cada $A \in \mathcal{L}\left(X_{1}, \ldots, X_{m} ; Y\right)$ definimos:

$$
\|A\|=\sup \left\{\|A(x)\|:\|x\|_{\infty} \leq 1, x \in X_{1} \times \ldots \times X_{m}\right\} .
$$

Na proposição apresentada a seguir estudamos algumas propriedades de $\|A\|$.

Proposição 2.1.3. Sejam $X_{1}, \ldots, X_{m}, Y$ espaços normados sobre o corpo $\mathbb{K} e$ $A \in \mathcal{L}\left(X_{1}, \ldots, X_{m} ; Y\right)$, então valem as seguintes afirmações:

1. $\|A(x)\| \leq\|A\|\left\|x_{1}\right\| \ldots\left\|x_{m}\right\|, \quad x=\left(x_{1}, \ldots, x_{m}\right) \in X_{1} \times \ldots \times X_{m}$;

2. \|A $\|$ é uma norma de $\mathcal{L}\left(X_{1}, \ldots, X_{m} ; Y\right)$;

3. $\|A\|=\inf \left\{M>0:\|A(x)\| \leq M\left\|x_{1}\right\| \ldots\left\|x_{m}\right\|\right.$, $\left.x=\left(x_{1}, \ldots, x_{m}\right) \in X_{1} \times \ldots \times X_{m}\right\}$; 
Demonstração: 1) Se existir $i=1, \ldots, m$ tal que $x_{i}=0$ então vale (1). Seja $x=\left(x_{1}, \ldots, x_{m}\right) \in X_{1} \times \ldots \times X_{m}$ tal que $x_{i} \neq 0$ para cada $i=1, \ldots, m$. Definimos, para cada $i=1, \ldots, m, z_{i}=\frac{x_{i}}{\left\|x_{i}\right\|}$. Então $\|z\|=1$, onde $z=\left(z_{1}, \ldots, z_{m}\right)$. Como

$$
\|A(z)\|=\frac{1}{\left\|x_{1}\right\| \ldots\left\|x_{m}\right\|}\|A(x)\|,
$$

temos que

$$
\|A(x)\|=\|A(z)\|\left\|x_{1}\right\| \ldots\left\|x_{m}\right\| \leq\|A\|\left\|x_{1}\right\| \ldots\left\|x_{m}\right\| .
$$

Portanto $\|A(x)\| \leq\|A\|\left\|x_{1}\right\| \ldots\left\|x_{m}\right\|$.

2) Da definição de $\|A\|$ segue que $\|A\| \geq 0$. Suponhamos que $\|A\|=0$. Então, para qualquer $x=\left(x_{1}, \ldots, x_{m}\right) \in X_{1} \times \ldots \times X_{m}$, temos que

$\|A(x)\| \leq\|A\|\left\|x_{1}\right\| \ldots\left\|x_{m}\right\|=0$. Assim $\|A(x)\|=0$ para qualquer $x \in X_{1} \times \ldots \times X_{m}$, isto é, $A \equiv 0$. Logo $\|A\|=0$ se e somente se $A=0$.

Seja $\alpha \in \mathbb{K}$. Então

$$
\|\alpha A\|=\sup \{\|\alpha A(x)\|:\|x\| \leq 1\}=|\alpha| \sup \{\|A(x)\|:\|x\| \leq 1\}=|\alpha|\|A\| .
$$

Portanto $\|\alpha A\|=|\alpha|\|A\|$.

Sejam $A_{1}, A_{2} \in \mathcal{L}\left(X_{1}, \ldots, X_{m} ; Y\right)$. Então, para qualquer $x \in X_{1} \times \ldots \times X_{m}$ com $\|x\| \leq 1$, temos que

$$
\left\|A_{1}(x)+A_{2}(x)\right\| \leq\left\|A_{1}(x)\right\|+\left\|A_{2}(x)\right\| \leq\left\|A_{1}\right\|+\left\|A_{2}\right\| .
$$

Portanto $\left\|A_{1}+A_{2}\right\| \leq\left\|A_{1}\right\|+\left\|A_{2}\right\|$.

3) Seja $M>0$ tal que para qualquer $x=\left(x_{1}, \ldots, x_{m}\right) \in X_{1} \times \ldots \times X_{m}$ temos que

$$
\left\|A\left(x_{1}, \ldots, x_{m}\right)\right\| \leq M\left\|x_{1}\right\| \ldots\left\|x_{m}\right\| .
$$

Agora consideremos qualquer $x=\left(x_{1}, \ldots, x_{m}\right) \in X_{1} \times \ldots \times X_{m}$ tal que $\|x\| \leq 1$. Então $\|A(x)\| \leq M$, isto é, M é uma cota superior de $\|A(x)\|$ para $\|x\| \leq 1$, consequentemente $\|A\| \leq M$. Portanto $\|A\|$ é uma cota inferior do conjunto

$$
\left\{M>0:\|A(x)\| \leq M\left\|x_{1}\right\| \ldots\left\|x_{m}\right\|, x=\left(x_{1}, \ldots, x_{m}\right) \in X_{1} \times \ldots \times X_{m}\right\},
$$

e pelo item (1) obtemos que $\|A\|$ é o ínfimo.

Na proposição acima vimos que $\mathcal{L}\left(X_{1}, \ldots, X_{m} ; Y\right)$ é um espaço normado se $X_{1}, \ldots, X_{m}$ e $Y$ são espaços normados sobre um corpo IK. No próximo teorema vamos estudar uma condição para que $\mathcal{L}\left(X_{1}, \ldots, X_{m} ; Y\right)$ seja um espaço de Banach. 
Teorema 2.1.4. Sejam $X_{1}, \ldots, X_{m}$, espaços normados e $Y$ um espaço de Banach sobre o corpo $\mathbb{I K}$ então $\mathcal{L}\left(X_{1}, \ldots, X_{m} ; Y\right)$ é um espaço de Banach.

Demonstração: Da proposição $2.1 .3 \mathcal{L}\left(X_{1}, \ldots, X_{m} ; Y\right)$ é um espaço normado, assim basta mostrar que é completo. Para tal seja $\left(A_{n}\right)_{n}$ uma sequência de Cauchy em $\mathcal{L}\left(X_{1}, \ldots, X_{m} ; Y\right)$. Então dado $\epsilon>0$ existe $N>0$ tal que para quaisquer $p, r>N$

$$
\left\|A_{p}-A_{r}\right\|<\epsilon
$$

Para cada $x=\left(x_{1}, \ldots, x_{m}\right) \in X_{1} \times \ldots \times X_{m}$ temos que

$$
\begin{aligned}
\left\|A_{p}(x)-A_{r}(x)\right\| & =\left\|\left(A_{p}-A_{r}\right)(x)\right\| \\
& \leq\left\|A_{p}-A_{r}\right\|\left\|x_{1}\right\| \ldots\left\|x_{m}\right\|,
\end{aligned}
$$

segue que $\left(A_{n}(x)\right)_{n}$ é uma sequência de Cauchy em $Y$ para qualquer $x=\left(x_{1}, \ldots, x_{m}\right) \in X_{1} \times \ldots \times X_{m}$. Como $Y$ é um espaço de Banach, a sequência será convergente. Então podemos definir uma aplicação m-linear dada por

$$
A(x)=\lim _{n \rightarrow \infty} A_{n}(x)
$$

para cada $x=\left(x_{1}, \ldots, x_{m}\right) \in X_{1} \times \ldots \times X_{m}$. É facil ver que A é m-linear. Vamos provar que $A$ é contínua em $x=\left(x_{1}, \ldots, x_{m}\right) \in X_{1} \times \ldots \times X_{m}$. Dado $\epsilon^{*}>0$ existe $n_{0} \in \mathbb{N}$ tal que, para qualquer $n>n_{0},\left\|A(x)-A_{n}(x)\right\|<\epsilon^{*}$, ou seja,

$$
\|A(x)\|<\epsilon^{*}+\left\|A_{n}(x)\right\| \text {. }
$$

Como $\left(A_{n}\right)_{n}$ é uma sequência de Cauchy, existe $M>0$ tal que $\left\|A_{n}\right\| \leq M$ para qualquer $n \in \mathbb{N}$. Então

$$
\|A(x)\|<\epsilon^{*}+M\left\|x_{1}\right\| \ldots\left\|x_{m}\right\|
$$

para qualquer $\epsilon^{*}>0$. Assim $\|A(x)\| \leq M\left\|x_{1}\right\| \ldots\left\|x_{m}\right\|$ para qualquer $x=\left(x_{1}, \ldots, x_{m}\right) \in X_{1} \times \ldots \times X_{m}$. Logo pelo teorema 2.1 .2 concluímos que $A \in \mathcal{L}\left(X_{1}, \ldots, X_{m} ; Y\right)$. Para $p$ suficientemente grande e $n>n_{0}$ fixo, segue de 2.1 e 2.2 que

$$
\left\|A(x)-A_{n}(x)\right\| \leq \epsilon\left\|x_{1}\right\| \ldots\left\|x_{m}\right\| .
$$

Portanto $\left\|A-A_{n}\right\| \leq \epsilon$ para todo $n>n_{0}$ e consequentemente $\mathcal{L}\left(X_{1}, \ldots, X_{m} ; Y\right)$ é completo.

No que segue, damos alguns exemplos de aplicações multilineares contínuas.

Exemplo 2.1.5. Sejam $X, Y, Z$ espaços normados. A aplicação

$$
A: \mathcal{L}(X ; Y) \times \mathcal{L}(Y ; Z) \longrightarrow \mathcal{L}(X ; Z)
$$

dada por $A(f, g)=g \circ f$ é bilinear e contínua. 
De fato: É facil mostrar que A é bilinear. Vamos provar que A é contínua. Sejam $f \in \mathcal{L}(X ; Y), g \in \mathcal{L}(Y ; Z)$, então para qualquer $x \in X$ tal que $\|x\| \leq 1$, temos que $\|g \circ f(x)\|=\|g(f(x))\| \leq\|g\|\|f\|\|x\| \leq\|g\|\|f\|$. Assim, $\|A(f, g)\|=\|g \circ f\| \leq\|g\| \cdot\|f\|$. Portanto $A$ é contínua.

Exemplo 2.1.6. Seja $X=l_{p}$ munido da norma usual. Dado $m \in \mathbb{N}$, seja $A: X^{m} \longrightarrow \mathbb{K}$ definida por:

$$
A\left(x_{1}, \ldots, x_{m}\right)=x_{11} \cdot x_{22} \cdot \ldots \cdot x_{m m}
$$

onde $x_{j}=\left(x_{j 1}, x_{j 2}, \ldots\right)$. A nada mais é que o produto dos elementos da diagonal. Afirmamos que $A$ é m-linear e contínua.

De fato: Claramente vemos que $A$ é m-linear. Seja $x=\left(x_{1}, \ldots x_{m}\right) \in X^{m}$, com $x_{i}=\left(x_{i 1}, x_{i 2}, \ldots\right)$, então:

$$
\left\|A\left(x_{1}, \ldots, x_{m}\right)\right\|=\left|x_{11} \cdot \ldots \cdot x_{m m}\right|=\left|x_{11}\right| \ldots\left|x_{m m}\right| \leq\left\|x_{1}\right\| \ldots\left\|x_{m}\right\|,
$$

e consequentemente $A$ é contínua.

No próximo teorema vamos estudar o isomorfismo isométrico entre os espaços $\mathcal{L}\left(X_{1}, \ldots, X_{m} ; Y\right)$ e $\mathcal{L}\left(X_{1} ; \mathcal{L}\left(X_{2}, \ldots, X_{m} ; Y\right)\right)$.

Teorema 2.1.7. Sejam $X_{1}, \ldots, X_{m}, Y$ espaços normados. Então o espaço $\mathcal{L}\left(X_{1}, \ldots, X_{m} ; Y\right)$ é isometricamente isomorfo ao espaço $\mathcal{L}\left(X_{1} ; \mathcal{L}\left(X_{2}, \ldots, X_{m} ; Y\right)\right)$.

Demonstração: Vamos definir $\psi: \mathcal{L}\left(X_{1}, \ldots, X_{m} ; Y\right) \longrightarrow \mathcal{L}\left(X_{1} ; \mathcal{L}\left(X_{2}, \ldots, X_{m} ; Y\right)\right)$ por:

$$
\psi(A)\left(x_{1}\right)\left(x_{2}, \ldots, x_{m}\right)=A\left(x_{1}, \ldots, x_{m}\right)
$$

para cada $x_{1} \in X_{1}$ e $\left(x_{2}, \ldots, x_{m}\right) \in X_{2} \times \ldots \times X_{m}$. A aplicação $\psi$ assim definida é um isomorfismo isométrico. De fato:

Sejam $A, B \in \mathcal{L}\left(X_{1}, \ldots, X_{m} ; Y\right), x_{i} \in X_{i}, i=1, \ldots, m$ e $\lambda \in \mathbb{K}$ :

$$
\begin{aligned}
\psi(A+\lambda B)\left(x_{1}\right)\left(x_{2}, \ldots, x_{m}\right) & =(A+\lambda B)\left(x_{1}, \ldots, x_{m}\right) \\
& =A\left(x_{1}, \ldots, x_{m}\right)+\lambda B\left(x_{1}, \ldots, x_{m}\right) \\
& =\psi(A)\left(x_{1}\right)\left(x_{2}, \ldots, x_{m}\right)+\lambda \psi(B)\left(x_{1}\right)\left(x_{2}, \ldots, x_{m}\right) .
\end{aligned}
$$

Logo

$$
\psi(A+\lambda B)=\psi(A)+\lambda \psi(B)
$$


Seja $B \in \mathcal{L}\left(X_{1} ; \mathcal{L}\left(X_{2}, \ldots, X_{m} ; Y\right)\right)$, definamos $A \in \mathcal{L}\left(X_{1}, \ldots, X_{m} ; Y\right)$ por:

$$
A\left(x_{1}, \ldots, x_{m}\right)=B\left(x_{1}\right)\left(x_{2}, \ldots, x_{m}\right) .
$$

para cada $\left(x_{1}, \ldots, x_{m}\right) \in X_{1} \times \ldots \times X_{m} . A$ assim definida é m-linear e contínua uma vez que $B\left(x_{1}\right) \in \mathcal{L}\left(X_{2}, \ldots, X_{m} ; Y\right), B \in \mathcal{L}\left(X_{1} ; \mathcal{L}\left(X_{2}, \ldots, X_{m} ; Y\right)\right)$ e

$$
\begin{aligned}
\left\|A\left(x_{1}, \ldots, x_{m}\right)\right\| & =\left\|B\left(x_{1}\right)\left(x_{2}, \ldots, x_{m}\right)\right\| \leq\left\|B\left(x_{1}\right)\right\|\left\|x_{2}\right\| \ldots\left\|x_{m}\right\| \\
& \leq\|B\|\left\|x_{1}\right\|\left\|x_{2}\right\| \ldots\left\|x_{m}\right\| .
\end{aligned}
$$

Assim, para cada $B \in \mathcal{L}\left(X_{1} ; \mathcal{L}\left(X_{2}, \ldots, X_{m} ; Y\right)\right)$, encontramos $A \in \mathcal{L}\left(X_{1}, \ldots, X_{m} ; Y\right)$ como acima tal que

$$
\psi(A)\left(x_{1}\right)\left(x_{2}, \ldots, x_{m}\right)=A\left(x_{1}, \ldots, x_{m}\right)=B\left(x_{1}\right)\left(x_{2}, \ldots, x_{m}\right),
$$

ou seja $\psi(A)=B$. Portanto $\psi$ é sobrejetora. Agora, para cada $A \in \mathcal{L}\left(X_{1}, \ldots, X_{m} ; Y\right)$,

$$
\|\psi(A)\|=\sup _{\left\|x_{1}\right\| \leq 1}\left\|\psi(A)\left(x_{1}\right)\right\|=\sup _{\left\|x_{i}\right\| \leq 1: 1 \leq i \leq m}\left\|A\left(x_{1}, \ldots, x_{m}\right)\right\|=\|A\| .
$$

Portanto $\psi$ é uma isometria.

Desta maneira, identificamos $\mathcal{L}\left(X_{1}, \ldots, X_{m} ; Y\right) \approx \mathcal{L}\left(X_{1} ; \mathcal{L}\left(X_{2}, \ldots, X_{m} ; Y\right)\right)$.

Corolário 2.1.8. Sejam $X, Y$ espaços normados, $m \in \mathbb{N}$. Então o espaço $\mathcal{L}\left({ }^{m} X ; Y\right)$ é isométricamente isomorfo ao espaço $\mathcal{L}\left(X ; \mathcal{L}\left({ }^{m-1} X ; Y\right)\right)$.

Demonstração: Consequência imediata do teorema acima.

Corolário 2.1.9. Sejam $X_{1}, \ldots, X_{m}, Y$ espaços normados. Então o espaço $\mathcal{L}\left(X_{1}, \ldots, X_{m} ; Y\right)$ é isométrico ao espaço $\mathcal{L}\left(X_{1} ; \mathcal{L}\left(X_{2} ; \ldots ; \mathcal{L}\left(X_{m} ; Y\right) \ldots\right)\right)$.

Demonstração: Segue direto do teorema 2.1.7.

$\mathcal{L}\left(X_{1}, \ldots, X_{m} ; Y\right) \approx \mathcal{L}\left(X_{1} ; \mathcal{L}\left(X_{2}, \ldots, X_{m} ; Y\right)\right) \approx \mathcal{L}\left(X_{1} ; \mathcal{L}\left(X_{2} ; \mathcal{L}\left(X_{3}, \ldots, X_{m} ; Y\right)\right)\right)$ $\approx \cdots \approx \mathcal{L}\left(X_{1} ; \mathcal{L}\left(X_{2} ; \ldots ; \mathcal{L}\left(X_{m} ; Y\right) \ldots\right)\right)$.

\subsection{Fórmula multinomial}

Nesta seção temos por objetivo estudar a fórmula de Leibniz. Primeiramente estudamos as aplicações simétricas, a simetrização de uma aplicação e seus principais resultados 
que serão utilizados no estudo da fórmula de Leibniz.

Para cada $n \in \mathbb{N}$ denotamos por $S_{n}$ o grupo de todas as permutações $\sigma$ de $\{1, \ldots, n\}$.

Definição 2.2.1. Sejam $X, Y$ espaços normados sobre o corpo $\mathbb{K}$ e $A: X^{m} \longrightarrow Y$ uma aplicação m-linear. Dizemos que A é simétrica se para cada $\sigma \in S_{m}$ temos:

$$
A\left(x_{1}, \ldots, x_{m}\right)=A\left(x_{\sigma(1)}, \ldots, x_{\sigma(m)}\right),
$$

para qualquer $x=\left(x_{1}, \ldots, x_{m}\right) \in X^{m}$.

Denotamos por $\left.\mathcal{L}_{a s}{ }^{m} X ; Y\right)$ o espaço vetorial de todas as aplicações m-lineares simétricas de $X^{m}$ em $Y$ e $\mathcal{L}_{s}\left({ }^{m} X ; Y\right)$ o espaço vetorial de todas as aplicações $\mathrm{m}$-lineares simétricas contínuas de $X^{m}$ em $Y$. Assim vemos que $\mathcal{L}_{s}\left({ }^{m} X ; Y\right)=\mathcal{L}\left({ }^{m} X ; Y\right) \cap \mathcal{L}_{a s}\left({ }^{m} X ; Y\right)$. Se $m=0, \mathcal{L}_{a s}\left({ }^{m} X ; Y\right)=\mathcal{L}_{s}\left({ }^{m} X ; Y\right)=Y$. Se $Y=\mathbb{K}, \mathcal{L}_{a s}\left({ }^{m} X ; Y\right)=\mathcal{L}_{a s}\left({ }^{m} X\right) \mathrm{e}$ $\mathcal{L}_{s}\left({ }^{m} X ; Y\right)=\mathcal{L}_{s}\left({ }^{m} X\right)$.

Proposição 2.2.2. Sejam $X, Y$ espaços normados sobre o corpo $\mathbb{I K}$. Então $\mathcal{L}_{s}\left({ }^{m} X ; Y\right)$ é um subespaço fechado de $\mathcal{L}\left({ }^{m} X ; Y\right)$.

Demonstração: Basta mostrar que $\overline{\mathcal{L}_{s}\left({ }^{m} X ; Y\right)} \subset \mathcal{L}_{s}\left({ }^{m} X ; Y\right)$. Seja $A \in \overline{\mathcal{L}_{s}\left({ }^{m} X ; Y\right)}$, então existe uma sequência $\left(A_{n}\right)_{n} \subset \mathcal{L}_{s}\left({ }^{m} X ; Y\right)$ tal que $A_{n} \stackrel{n \rightarrow \infty}{\longrightarrow} A$. Vamos mostrar que $A$ é simétrica. Para cada $x=\left(x_{1}, \ldots, x_{m}\right) \in X^{m}$ e para cada $\sigma \in S_{m}$ :

$$
A\left(x_{1}, \ldots, x_{m}\right)=\lim _{n \rightarrow \infty} A_{n}\left(x_{1}, \ldots, x_{m}\right)=\lim _{n \rightarrow \infty} A_{n}\left(x_{\sigma(1)}, \ldots, x_{\sigma(m)}\right),
$$

pois $A_{n}$ é simétrica para cada $n \in \mathbb{N}$. Assim

$$
A\left(x_{1}, \ldots, x_{m}\right)=A\left(x_{\sigma(1)}, \ldots, x_{\sigma(m)}\right),
$$

e consequentemente, $A \in \mathcal{L}_{s}\left({ }^{m} X ; Y\right)$.

Proposição 2.2.3. Sejam $X$ um espaço normado, $Y$ um espaço de Banach sobre o corpo $\mathbb{K}$ e $m \in \mathbb{N}$. Então $\mathcal{L}_{s}\left({ }^{m} X ; Y\right)$ é um espaço de Banach.

Demonstração: Pelo teorema 2.1.4 temos que o espaço $\mathcal{L}\left({ }^{m} X ; Y\right)$ é um espaço de Banach. Pela proposição $2.2 .2, \mathcal{L}_{s}\left({ }^{m} X ; Y\right)$ é um espaço fechado de $\mathcal{L}\left({ }^{m} X ; Y\right)$. Portanto, $\mathcal{L}_{s}\left({ }^{m} X ; Y\right)$ é um espaço de Banach.

O próximo teorema nos dará a simetrização de uma aplicação m-linear. 
Teorema 2.2.4. Sejam $X, Y$ espaços normados e $m \in \mathbb{N}$. Para cada aplicação m-linear $A: X^{m} \longrightarrow Y$ definimos a aplicação $A_{s}: X^{m} \longrightarrow Y$ por:

$$
A_{s}\left(x_{1}, \ldots, x_{m}\right)=\frac{1}{m !} \sum_{\sigma \in S_{m}} A\left(x_{\sigma(1)}, \ldots, x_{\sigma(m)}\right)
$$

então $A_{s}$ é uma aplicação m-linear simétrica e $\left\|A_{s}\right\| \leq\|A\|$.

Demonstração: $A_{s}$ é m-linear pois é soma de aplicações m-lineares. Seja $\pi \in S_{m}$ e seja $x_{i} \in X i=1, \ldots, m$ :

$$
\begin{aligned}
A_{s}\left(x_{\pi(1)}, \ldots, x_{\pi(m)}\right) & =\frac{1}{m !} \sum_{\sigma \in S_{m}} A\left(x_{\sigma(\pi(1))}, \ldots, x_{\sigma(\pi(m))}\right) \\
& =\frac{1}{m !} \sum_{\beta \in S_{m}} A\left(x_{\beta(1)}, \ldots, x_{\beta(m)}\right) \\
& =A_{s}\left(x_{1}, \ldots, x_{m}\right) .
\end{aligned}
$$

Logo, $A_{s}$ é simétrica. Considere

$$
\begin{aligned}
\left\|A_{s}\right\| & =\sup _{\left\|x_{i}\right\| \leq 1: 1 \leq i \leq m}\left\|A_{s}\left(x_{1}, \ldots, x_{m}\right)\right\| \\
& =\sup _{\left\|x_{i}\right\| \leq 1: 1 \leq i \leq m}\left\|\frac{1}{m !} \sum_{\sigma \in S_{m}} A\left(x_{\sigma(1)}, \ldots, x_{\sigma(m)}\right)\right\| .
\end{aligned}
$$

Se $\left\|x_{i}\right\| \leq 1$, para $1 \leq i \leq m$, temos que $\left\|A\left(x_{\sigma(1)}, \ldots, x_{\sigma(m)}\right)\right\| \leq\|A\|$. Assim,

$$
\begin{aligned}
& \sup _{\left\|x_{i}\right\| \leq 1: 1 \leq i \leq m}\left\|\frac{1}{m !} \sum_{\sigma \in S_{m}} A\left(x_{\sigma(1)}, \ldots, x_{\sigma(m)}\right)\right\| \leq \\
& \sup _{\left\|x_{i}\right\| \leq 1: 1 \leq i \leq m} \frac{1}{m !} \sum_{\sigma \in S_{m}}\left\|A\left(x_{\sigma(1)}, \ldots, x_{\sigma(m)}\right)\right\| \leq\|A\| .
\end{aligned}
$$

Portanto $\left\|A_{s}\right\| \leq\|A\|$.

Definição 2.2.5. Sejam $X, Y$ espaços normados sobre o corpo $\mathbb{K}$ e $m \in \mathbb{N}$. Seja $A: X^{m} \longrightarrow Y$ uma aplicação m-linear. A aplicação $A_{s}$ definida no teorema 2.2.4 será chamada de simetrização de $A$.

Observamos que se $A \in \mathcal{L}_{s}\left({ }^{m} X ; Y\right), X, Y$ espaços normados sobre o corpo $\mathbb{K}$ e $m \in \mathbb{N}$, então temos que $A_{s}=A$. De fato: Para cada $i=1, \ldots, m$, seja $x_{i} \in X$. Para cada $\sigma \in S_{m}$ 
temos que $A\left(x_{\sigma(1)}, \ldots, x_{\sigma(m)}\right)=A\left(x_{1}, \ldots, x_{m}\right)$ pois $A \in \mathcal{L}_{s}\left({ }^{m} X ; Y\right)$. Assim,

$$
A_{s}\left(x_{1}, \ldots, x_{m}\right)=\frac{1}{m !} \sum_{\sigma \in S_{m}} A\left(x_{\sigma(1)}, \ldots, x_{\sigma(m)}\right)=\frac{1}{m !} \sum_{\sigma \in S_{m}} A\left(x_{1}, \ldots, x_{m}\right)=A\left(x_{1}, \ldots, x_{m}\right) \text {. }
$$

Assim, se considerarmos a aplicação $\phi: \mathcal{L}\left({ }^{m} X ; Y\right) \longrightarrow \mathcal{L}_{s}\left({ }^{m} X ; Y\right)$ definida por $\phi(A)=A_{s}$ para cada $A \in \mathcal{L}\left({ }^{m} X ; Y\right)$. Facilmente vemos que $\phi$ é linear contínua e sobrejetora.

Quando $A \in \mathcal{L}_{a}\left({ }^{m} X ; Y\right)$ e $x=x_{1}=\ldots=x_{n}$ vamos denotar $A\left(x_{1}, \ldots, x_{n}\right)$ por $A x^{n}$. Se $\mathrm{n}=0, \mathrm{~A} x^{0}=\mathrm{A}$.

Proposição 2.2.6. Sejam $X, Y$ espaços vetoriais, $m \in \mathbb{N}, A \in \mathcal{L}_{a}\left({ }^{m} X ; Y\right), k \in \mathbb{N}$. Para cada $x_{1}, \ldots, x_{k} \in X$ e cada $n_{1}, \ldots, n_{k} \in \mathbb{N}$ com $n_{1}+\ldots+n_{k}=n \leq m$. Definimos a aplicação $A\left(x_{1}^{n_{1}} \ldots x_{k}^{n_{k}}\right): X^{m-n} \longrightarrow Y$ por:

$A\left(x_{1}^{n_{1}} \ldots x_{k}^{n_{k}}\right)\left(x_{n+1}, \ldots, x_{m}\right)=\left\{\begin{array}{cll}A\left(x_{1}, \stackrel{\left(n_{1}\right)}{*}, x_{1}, \ldots, x_{k}, \stackrel{\left(n_{k}\right)}{\cdots}, x_{k}, x_{n+1}, \ldots, x_{m}\right) & \text { se } & n \geq 1 \\ A\left(x_{1}, \ldots, x_{m}\right) & \text { se } & n=0\end{array}\right.$

então $A\left(x_{1}^{n_{1}} \ldots x_{k}^{n_{k}}\right)$ é $(m-n)$-linear.

Demonstração: Facilmente vemos que $A\left(x_{1}^{n_{1}} \ldots x_{k}^{n_{k}}\right)$ é $(\mathrm{m}-\mathrm{n})$-linear, pois segue da m-linearidade da aplicação $A$.

No que segue vamos demonstrar um teorema que nos dá a fórmula multinomial, conhecida como Fórmula de Leibniz. Como Leonardo Pellegrini em sua dissertação de mestrado, [[28], teorema 1.13 pg 18], redigiu tal demonstração de maneira muito clara, decidimos aqui apresentar a demostração redigida por ele. Para isso, tal demonstração precisa da seguinte notação: para cada $k \in \mathbb{N}$ e cada $\alpha=\left(n_{1}, \ldots, n_{k}\right) \in \mathbb{N}^{k}$, definimos

$$
|\alpha|=n_{1}+\ldots+n_{k} \quad \text { e } \quad \alpha !=n_{1} ! \ldots n_{k} !
$$

\section{Teorema 2.2.7. (Fórmula de Leibniz)}

Sejam $X, Y$ espaços vetoriais sobre o corpo $\mathbb{K}, m \in \mathbb{N}$ e $A \in \mathcal{L}_{a s}\left({ }^{m} X ; Y\right)$. Então, para todo $x_{1}, \ldots, x_{k} \in X$ temos que

$$
A\left(x_{1}+\ldots+x_{k}\right)^{m}=\sum_{|\alpha|=m} \frac{m !}{n_{1} ! \ldots n_{k} !} A\left(x_{1}^{n_{1}} \ldots x_{k}^{n_{k}}\right) .
$$


onde a soma é sobre todas as $k$-uplas $\alpha=\left(n_{1}, \ldots, n_{k}\right) \in \mathbb{N}^{k}$ satisfazendo $n_{1}+\ldots+n_{k}=m$.

Demonstração: Para $m=0$ e $m=1$ o resultado é trivial. Assumindo a fórmula válida para $m \geq 1$, seja $A \in \mathcal{L}_{a s}\left({ }^{m+1} X ; Y\right)$. Podemos escrever

$$
A\left(x_{1}+\ldots+x_{k}\right)^{m+1}=A\left(x_{1}+\ldots+x_{k}\right)\left(x_{1}+\ldots+x_{k}\right)^{m} .
$$

Aplicando a hipótese de indução para a $m$-linear simétrica $A\left(x_{1}+\ldots+x_{k}\right)$, obtemos

$$
\begin{gathered}
A\left(x_{1}+\ldots+x_{k}\right)^{m+1}=\sum_{|\alpha|=m} \frac{m !}{n_{1} ! \ldots n_{k} !} A\left(x_{1}+\ldots+x_{k}\right)\left(x_{1}^{n_{1}} \ldots x_{k}^{n_{k}}\right) \\
\quad=\sum_{|\alpha|=m} \frac{m !}{n_{1} ! \ldots n_{k} !} A\left(x_{1}^{n_{1}+1} \ldots x_{k}^{n_{k}}\right)+ \\
+\sum_{|\alpha|=m} \frac{m !}{n_{1} ! \ldots n_{k} !} A\left(x_{1}^{n_{1}} x_{2}^{n_{2}+1} \ldots x_{k}^{n_{k}}\right)+ \\
+\ldots+\sum_{|\alpha|=m} \frac{m !}{n_{1} ! \ldots n_{k} !} A\left(x_{1}^{n_{1}} \ldots x_{k}^{n_{k}+1}\right),
\end{gathered}
$$

onde $\alpha=\left(n_{1}, \ldots, n_{k}\right) \in \mathbb{N}^{k}$ tal que $|\alpha|=m$.

Para cada $i=1, \ldots, k$, definimos $\beta^{[i]}=\left(\beta_{1}^{[i]}, \ldots, \beta_{k}^{[i]}\right) \in \mathbb{N}^{k}$ tal que $\beta_{i}^{[i]}=n_{i}+1$ e $\beta_{j}^{[i]}=n_{j}$ para $j \neq i, j=1, \ldots, k$. Assim,

$$
\begin{gathered}
A\left(x_{1}+\ldots+x_{k}\right)^{m+1}=\sum_{\left|\beta^{[1]}\right|=m+1} \frac{m !}{\left(\beta_{1}^{[1]}-1\right) ! \beta_{2}^{[1]} ! \ldots \beta_{k}^{[1]} !} A\left(x_{1}^{\beta_{1}^{[1]}} \ldots x_{k}^{\beta_{k}^{[1]}}\right)+\ldots \\
\ldots+\sum_{\left|\beta^{[k]}\right|=m+1} \frac{m !}{\left(\beta_{1}^{[k]} ! \beta_{2}^{[k]} ! \ldots\left(\beta_{k}^{[k]}-1\right) !\right.} A\left(x_{1}^{\beta_{1}^{[k]}} \ldots x_{k}^{\beta_{k}^{[k]}}\right) \\
=\sum_{|\beta|=m+1 ; \beta_{1} \geq 1} \frac{m !}{\left(\beta_{1}-1\right) ! \beta_{2} ! \ldots \beta_{k} !} A\left(x_{1}^{\beta_{1}} \ldots x_{k}^{\beta_{k}}\right)+\ldots \\
\ldots+\sum_{|\beta|=m+1 ; \beta_{m} \geq 1} \frac{m !}{\beta_{1} ! \beta_{2} ! \ldots\left(\beta_{k}-1\right) !} A\left(x_{1}^{\beta_{1}} \ldots x_{k}^{\beta_{k}}\right) \\
\quad \sum_{|\beta|=m+1 ; \beta_{1} \geq 1} \frac{m ! \beta_{1}}{\beta_{1} ! \beta_{2} ! \ldots \beta_{k} !} A\left(x_{1}^{\beta_{1}} \ldots x_{k}^{\beta_{k}}\right)+\ldots \\
\quad \ldots+\sum_{|\beta|=m+1 ; \beta_{m} \geq 1} \frac{m ! \beta_{k}}{\beta_{1} ! \beta_{2} ! \ldots \beta_{k} !} A\left(x_{1}^{\beta_{1}} \ldots x_{k}^{\beta_{k}}\right),
\end{gathered}
$$


onde $\beta=\left(\beta_{1}, \ldots, \beta_{k}\right) \in \mathbb{N}^{k}$. Logo

$$
\begin{gathered}
A\left(x_{1}+\ldots+x_{k}\right)^{m+1}=\sum_{|\beta|=m+1} m ! A\left(x_{1}^{\beta_{1}} \ldots x_{k}^{\beta_{k}}\right)\left(\frac{\beta_{1}}{\beta_{1} ! \beta_{2} ! \ldots \beta_{k} !}+\ldots+\frac{\beta_{k}}{\beta_{1} ! \beta_{2} ! \ldots \beta_{k} !}\right) \\
=\sum_{|\beta|=m+1} \frac{m !}{\beta_{1} ! \beta_{2} ! \ldots \beta_{k} !} A\left(x_{1}^{\beta_{1}} \ldots x_{k}^{\beta_{k}}\right)\left(\beta_{1}+\ldots+\beta_{k}\right) \\
=\sum_{|\beta|=m+1} \frac{(m+1) !}{\beta !} A\left(x_{1}^{\beta_{1}} \ldots x_{k}^{\beta_{k}}\right),
\end{gathered}
$$

que é a fórmula que queríamos para $m+1$.

O próximo teorema nos mostra que uma aplicação multilinear simétrica depende apenas de seus valores na diagonal do espaço $X^{m}$. Ele será útil no estudo dos polinômios.

\section{Teorema 2.2.8. (Fórmula da Polarização)}

Sejam $X, Y$ espaços vetoriais sobre o corpo $\mathbb{K}, m \in \mathbb{N}$ e $A: X^{m} \longrightarrow Y$ uma aplicação m-linear simétrica. Então, para cada $x_{1}, \ldots, x_{m} \in X$, temos:

$$
A\left(x_{1}, \ldots, x_{m}\right)=\frac{1}{m ! 2^{m}} \sum_{\epsilon_{i}= \pm 1,1 \leq i \leq m} \epsilon_{1} \ldots \epsilon_{m} A\left(\epsilon_{1} x_{1}+\ldots+\epsilon_{m} x_{m}\right)^{m} .
$$

Demonstração: Pela fórmula de Leibniz (2.2.7), para cada sequência $\alpha=\left(n_{1}, \ldots, n_{m}\right) \in \mathbb{N}^{m} \operatorname{com}|\alpha|=n_{1}+\ldots+n_{m}=m$, temos:

$$
\begin{aligned}
A\left(\epsilon_{1} x_{1}+\ldots+\epsilon_{m} x_{m}\right)^{m} & =\sum_{|\alpha|} \frac{m !}{n_{1} ! \ldots n_{m} !} A\left(\left(\epsilon_{1} x_{1}\right)^{n_{1}} \ldots\left(\epsilon_{m} x_{m}\right)^{n_{m}}\right) \\
& =\sum_{|\alpha|} \frac{m !}{n_{1} ! \ldots n_{m} !} \epsilon_{1}^{n_{1}} \ldots \epsilon_{m}^{n_{m}} A\left(x_{1}^{n_{1}} \ldots x_{m}^{n_{m}}\right),
\end{aligned}
$$

onde a soma é sobre todas as m-uplas $\alpha=\left(n_{1}, \ldots, n_{m}\right) \in \mathbb{N}^{m}$ satisfazendo $|\alpha|=n_{1}+\ldots+n_{m}=m$. Assim,

$$
\begin{aligned}
& \frac{1}{m ! 2^{m}} \sum_{\epsilon_{i}= \pm 1,1 \leq i \leq m} \epsilon_{1} \ldots \epsilon_{m} A\left(\epsilon_{1} x_{1}+\ldots+\epsilon_{m} x_{m}\right)^{m} \\
& =\frac{1}{m ! 2^{m}} \sum_{\epsilon_{i}= \pm 1,1 \leq i \leq m} \epsilon_{1} \ldots \epsilon_{m} \sum_{|\alpha|} \frac{m !}{n_{1} ! \ldots n_{m} !} \epsilon_{1}^{n_{1}} \ldots \epsilon_{m}^{n_{m}} A\left(x_{1}^{n_{1}} \ldots x_{m}^{n_{m}}\right) \\
& =\frac{1}{2^{m}} \sum_{|\alpha|} \frac{1}{n_{1} ! \ldots n_{m} !} \sum_{\epsilon_{i}= \pm 1,1 \leq i \leq m} \epsilon_{1}^{n_{1}+1} \ldots \epsilon_{m}^{n_{m}+1} A\left(x_{1}^{n_{1}} \ldots x_{m}^{n_{m}}\right) .
\end{aligned}
$$


Se algum $n_{i}=0$, temos que:

$$
\begin{aligned}
\sum_{\epsilon_{i}= \pm 1,1 \leq i \leq m} \epsilon_{1}^{n_{1}+1} \ldots \epsilon_{m}^{n_{m}+1}= & \sum_{\epsilon_{j}= \pm 1,1 \leq j \leq m, j \neq i} \epsilon_{1}^{n_{1}+1} \ldots 1 \epsilon_{i+1}^{n_{i+1}+1} \ldots \epsilon_{m}^{n_{m}+1}+ \\
& \sum_{\epsilon_{j}= \pm 1,1 \leq j \leq m, j \neq i} \epsilon_{1}^{n_{1}+1} \ldots(-1) \epsilon_{i+1}^{n_{i+1}+1} \ldots \epsilon_{m}^{n_{m}+1} \\
= & (1-1) \sum_{\epsilon_{j}= \pm 1,1 \leq j \leq m, j \neq i} \epsilon_{1}^{n_{1}+1} \ldots \epsilon_{m}^{n_{m}+1}=0 .
\end{aligned}
$$

Assim, as parcelas que aparecerão no somatório é quando $n_{i} \neq 0$ para cada $i=1, \ldots, m$, e como $n_{1}+\ldots+n_{m}=m$, a única possibilidade é de $n_{i}=1$ para cada $i=1, \ldots, m$. Assim,

$$
\sum_{\epsilon_{i}= \pm 1,1 \leq i \leq m} \epsilon_{1}^{n_{1}+1} \ldots \epsilon_{m}^{n_{m}+1}=\sum_{\epsilon_{i}= \pm 1,1 \leq i \leq m} \epsilon_{1}^{2} \ldots \epsilon_{m}^{2}=2^{m} \cdot 1=2^{m} .
$$

Logo,

$$
\begin{aligned}
& \frac{1}{m ! 2^{m}} \sum_{\epsilon_{i}= \pm 1,1 \leq i \leq m} \epsilon_{1} \ldots \epsilon_{m} A\left(\epsilon_{1} x_{1}+\ldots+\epsilon_{m} x_{m}\right)^{m} \\
& =\frac{1}{2^{m}} \sum_{|\alpha|} \frac{1}{n_{1} ! \ldots n_{m} !} \sum_{\epsilon_{i}= \pm 1,1 \leq i \leq m} \epsilon_{1}^{n_{1}+1} \ldots \epsilon_{m}^{n_{m}+1} A\left(x_{1}^{n_{1}} \ldots x_{m}^{n_{m}}\right) \\
& =\frac{1}{2^{m}} \sum_{|\alpha|} \frac{1}{n_{1} ! \ldots n_{m} !} 2^{m} A\left(x_{1}^{n_{1}} \ldots x_{n}^{n_{m}}\right) \\
& =A\left(x_{1} \ldots x_{m}\right)=A\left(x_{1}, \ldots, x_{m}\right) .
\end{aligned}
$$

\subsection{Polinômios homogêneos}

O nosso objetivo nesta seção é definir os polinômios m-homogêneos e polinômios. Aqui vamos estudar a unicidade da correspondência entre o espaço dos polinômios mhomogêneos e o espaço das aplicações m-lineares simétricas, como também a unicidade da representação de um polinômio.

Definição 2.3.1. Sejam X, Y espaços normados sobre o mesmo corpo IK. Uma aplicação $P: X \longrightarrow Y$ é dita ser um polinômio $\mathrm{m}$-homogêneo se existe uma aplicação m-linear $A: X^{m} \longrightarrow Y$ tal que $P(x)=A x^{m}$ para todo $x \in X$. 
Exemplo 2.3.2. Sejam $n, p \in \mathbb{N}$ e $P: l_{p} \longrightarrow \mathbb{K}$ dado por:

$$
P(x)=\sum_{i=1}^{n} x_{j}^{2} \quad \operatorname{com} x=\left(x_{j}\right)_{j} \in l_{p}
$$

Afirmamos que P é um polinômio 2-homogêneo.

De fato: Tomemos $A: l_{p} \times l_{p} \longrightarrow \mathbb{K}$ dado por:

$$
A(x, y)=\sum_{i=1}^{n} x_{i} y_{i} \quad \operatorname{com} x=\left(x_{i}\right)_{i} \text { e } y=\left(y_{i}\right)_{i} \in l_{p}
$$

Facilmente vemos que $A$ é 2-linear e como

$$
A(x, x)=\sum_{i=1}^{n} x_{i} x_{i}=\sum_{i=1}^{n} x_{i}^{2}=P(x),
$$

temos que $P$ é 2-homogêneo.

Da definição, vemos que um polinômio m-homogêneo pode ser escrito da forma $P=A \circ \Delta_{m}$, onde $A \in \mathcal{L}_{a}\left({ }^{m} X ; Y\right)$ e $\Delta_{m}$ é a aplicação diagonal $\Delta_{m}: X \longrightarrow X^{m}$ dada por:

$$
\Delta_{m}(x)=(x, \stackrel{(m)}{\cdot}, x)
$$

Facilmente vemos que $\Delta_{m}$ é linear.

Observamos que se $P: X \longrightarrow Y$ é um polinômio m-homogêneo, então $P(\lambda x)=\lambda^{m} P(x)$ para todo $\lambda \in \mathbb{K}$ e todo $x \in X$, já que existe $A: X^{m} \longrightarrow Y$ m-linear com $P(x)=A x^{m}$ para todo $x \in X$.

Vamos denotar por $\mathcal{P}_{a}\left({ }^{m} X ; Y\right)$ o espaço vetorial de todos os polinômios $\mathrm{m}$-homogêneos de $X$ em $Y$. Se $m=0, \mathcal{P}_{a}\left({ }^{m} X ; Y\right)=Y$.

No próximo teorema vamos provar que se a aplicação m-linear $A$ for simétrica então $A$ é única.

Teorema 2.3.3. Sejam $X, Y$ espaços vetoriais sobre o corpo $\mathbb{I K}, m \in \mathbb{N}$. Dado $P \in \mathcal{P}_{a}\left({ }^{m} X ; Y\right)$ existe uma única aplicação m-linear simétrica $A_{s} \in \mathcal{L}_{a s}\left({ }^{m} X ; Y\right)$ tal que $P(x)=A_{s} x^{m}$ para cada $x \in X$. 
Demonstração: Dado $P \in \mathcal{P}_{a}\left({ }^{m} X ; Y\right)$, existe $A \in \mathcal{L}_{a}\left({ }^{m} X ; Y\right)$ tal que $P(x)=A x^{m}$ para cada $x \in X$. Tomando a simetrização de $A$, temos:

$$
\begin{aligned}
A_{s} x^{m} & =A_{s}(x, \stackrel{(m)}{!}, x)=\frac{1}{m !} \sum_{\sigma \in S_{m}} A(x, \stackrel{(m)}{*}, x) \\
& =\frac{1}{m !} \sum_{\sigma \in S_{m}} A x^{m}=A x^{m}=P(x)
\end{aligned}
$$

para qualquer $x \in X$. Para verificar a unicidade, suponhamos que existem $A_{1}, A_{2} \in \mathcal{L}_{a s}\left({ }^{m} X ; Y\right)$ tais que, para cada $x \in X$

$$
P(x)=A_{1} x^{m} \quad \text { e } \quad P(x)=A_{2} x^{m} .
$$

Assim, $A_{1} x^{m}=A_{2} x^{m}$, logo $\left(A_{1}-A_{2}\right) x^{m}=0$ para cada $x \in X$.

Segue da fórmula da polarização (2.2.8) que

$$
\begin{aligned}
\left(A_{1}-A_{2}\right)\left(x_{1}, \ldots, x_{m}\right) & =\frac{1}{m ! 2^{m}} \sum_{\epsilon_{i}= \pm 1,1 \leq i \leq m} \epsilon_{1} \ldots \epsilon_{m}\left(A_{1}-A_{2}\right)\left(\epsilon_{1} x_{1}+\ldots+\epsilon_{m} x_{m}\right)^{m} \\
& =\frac{1}{m ! 2^{m}} \sum_{\epsilon_{i}= \pm 1,1 \leq i \leq m} \epsilon_{1} \ldots \epsilon_{m} \cdot 0=0 .
\end{aligned}
$$

para cada $\left(x_{1}, \ldots, x_{m}\right) \in X^{m}$. Assim, $A_{1}\left(x_{1}, \ldots, x_{m}\right)=A_{2}\left(x_{1}, \ldots, x_{m}\right)$.

Portanto $A_{1}=A_{2}$.

Dado $P \in \mathcal{P}_{a}\left({ }^{m} X ; Y\right)$, segue dos teoremas 2.2 .8 e 2.3 .3 que existe uma única $A \in \mathcal{L}_{a s}\left({ }^{m} X ; Y\right)$ tal que

$$
A\left(x_{1}, \ldots, x_{m}\right)=\frac{1}{m ! 2^{m}} \sum_{\epsilon_{i}= \pm 1,1 \leq i \leq m} \epsilon_{1} \ldots \epsilon_{m} P\left(\epsilon_{1} x_{1}+\ldots+\epsilon_{m} x_{m}\right) .
$$

Esta única correspondência entre o polinômio $\mathrm{m}$-homogêneo $P$ e a aplicação $\mathrm{m}$-linear simétrica $A$ tal que $P(x)=A x^{m}$, vamos denotar por $P=\hat{A}$.

Usando o teorema 2.3 .3 podemos mostrar que os espaços $\mathcal{L}_{a s}\left({ }^{m} X ; Y\right)$ e $\mathcal{P}_{a}\left({ }^{m} X ; Y\right)$ são isomorfos.

Teorema 2.3.4. Sejam $X, Y$ espaços vetoriais sobre o corpo $\mathbb{K}$ e $m \in \mathbb{N}$. Então os espaços vetoriais $\mathcal{L}_{a s}\left({ }^{m} X ; Y\right)$ e $\mathcal{P}_{a}\left({ }^{m} X ; Y\right)$ são isomorfos.

Demonstração: $\quad$ Seja $\phi: \mathcal{L}_{a s}\left({ }^{m} X ; Y\right) \longrightarrow \mathcal{P}_{a}\left({ }^{m} X ; Y\right)$ dada por $\phi(A)=\hat{A}$ para cada $A \in \mathcal{L}_{a s}\left({ }^{m} X ; Y\right)$. Assim, $\phi$ é linear, uma vez que dado $A, B \in \mathcal{L}_{a s}\left({ }^{m} X ; Y\right), \lambda \in \mathbb{K}$ e $x \in X:$

$$
\phi(A+\lambda B)(x)=(\widehat{A+\lambda B})(x)=(A+\lambda B) x^{m}=A x^{m}+\lambda B x^{m}
$$




$$
=\hat{A}(x)+\lambda \hat{B}(x)=\phi(A)(x)+\lambda \phi(B)(x)=[\phi(A)+\lambda \phi(B)](x) .
$$

Pelo teorema anterior (2.3.3) segue que $\phi$ é uma bijeção.

Definição 2.3.5. Sejam $X, Y$ espaços vetoriais sobre o corpo $\mathbb{K}$. Uma aplicação $P: X \longrightarrow Y$ é dita ser um polinômio de grau $\mathrm{m}$ se existem $P_{k} \in \mathcal{P}_{a}\left({ }^{k} X ; Y\right)$, $k=0, \ldots, m$ tais que $P=P_{0}+\ldots+P_{m}$, isto é, $P(x)=P_{0}(x)+\ldots+P_{m}(x)$ para cada $x \in X$.

Vamos denotar por $\mathcal{P}_{a}(X ; Y)$ o espaço vetorial de todos os polinômios de $X$ em $Y$. Se $Y=\mathbb{K}$ então $\mathcal{P}_{a}(X ; Y)=\mathcal{P}_{a}(X)$.

No próximo teorema vamos demonstrar a unicidade da representação de $P$.

Teorema 2.3.6. Sejam $X, Y$ espaços vetoriais sobre o corpo $\mathbb{K}$. Seja $P: X \longrightarrow Y$ um polinômio não-nulo de grau $m$. Então a representação $P=P_{0}+\ldots+P_{m}$ é única.

Demonstração: Primeiramente vamos mostrar que se $R=R_{0}+\ldots+R_{m}=0$, então $R_{0}=R_{1}=\ldots=R_{m}=0$. Para quaisquer $x \in X$ e $\alpha \in \mathbb{K}, \alpha \neq 0$, temos que

$$
\begin{gathered}
R(\alpha x)=R_{0}(\alpha x)+R_{1}(\alpha x) \ldots+R_{m}(\alpha x)=0 \\
\Rightarrow \quad \alpha^{0} R_{0}(x)+\alpha^{1} R_{1}(x) \ldots+\alpha^{m} R_{m}(x)=0 \\
\stackrel{\dot{\alpha}^{m}}{\Rightarrow} \quad \frac{1}{\alpha^{m}} R_{0}(x)+\frac{\alpha}{\alpha^{m}} R_{1}(x) \ldots+\frac{\alpha^{m}}{\alpha^{m}} R_{m}(x)=0 .
\end{gathered}
$$

Para $\alpha$ suficientemente grande, segue que $R_{m}(x)=0$ para qualquer $x \in X$. Logo $R(x)=R_{0}(x)+R_{1}(x) \ldots+R_{m-1}(x)=0$. Com raciocínio análogo, concluímos que $R_{0}=R_{1}=\ldots=R_{m}=0$.

Agora consideremos um polinômio qualquer $P: X \longrightarrow Y$ não-nulo tal que

$$
P=P_{0}+\ldots+P_{m} \quad \text { e } \quad P=Q_{0}+\ldots+Q_{m}
$$

onde $P_{k}, Q_{k} \in \mathcal{P}_{a}\left({ }^{k} X ; Y\right), k=0, \ldots, m$. Então,

$$
\left(P_{0}-Q_{0}\right)+\left(P_{1}-Q_{1}\right)+\ldots+\left(P_{m}-Q_{m}\right)=0
$$

e pela parte anterior, temos que

$$
P_{0}-Q_{0}=P_{1}-Q_{1}=\ldots=P_{m}-Q_{m}=0 .
$$


$\operatorname{logo} P_{0}=Q_{0}, P_{1}=Q_{1}, \ldots P_{m}=Q_{m}$. Portanto, a representação de $P$ é única.

A seguir vamos definir a polarização de uma função $f: X \longrightarrow Y$ e estudar a polarização de um polinômio.

Definição 2.3.7. Sejam $X, Y$ espaços vetoriais sobre o corpo $\mathbb{K}$ e $m \in \mathbb{N}$. Sejam $f: X \longrightarrow Y$ e $x_{1}, \ldots, x_{m} \in X$. Definimos a aplicação $\phi_{m}$ do espaço das funções de $X$ em $Y$ no espaço $Y$ como sendo

$$
\phi_{m}(f)=\frac{1}{m ! 2^{m}} \sum_{\epsilon_{i} \pm 11 \leq i \leq m} \epsilon_{1} \cdot \ldots \cdot \epsilon_{m} f\left(\epsilon_{1} x_{1}+\ldots+\epsilon_{m} x_{m}\right) .
$$

Chamamos a aplicação $\phi_{m}$ de polarização de $\mathbf{f}$ com respeito a $x_{1}, \ldots, x_{m}$.

Teorema 2.3.8. Sejam $X, Y$ espaços de Banach sobre o corpo $\mathbb{K}$ e $m \in \mathbb{N}$. Se $A_{k}: X^{k} \longrightarrow Y$ é uma aplicação k-linear simétrica e $P_{k}=\hat{A}_{k}$, então:

$$
\phi_{m}\left(P_{k}\right)=\left\{\begin{array}{ccc}
A_{m}\left(x_{1}, \ldots, x_{m}\right) & \text { se } & k=m \\
0 & \text { se } & k<m
\end{array} .\right.
$$

Demonstração: Se $k=m$, segue da fórmula da polarização de $P_{m}$ com respeito a $x_{1}, \ldots, x_{m} \in X$ que,

$$
\begin{aligned}
\phi_{m}\left(P_{m}\right) & =\frac{1}{m ! 2^{m}} \sum_{\epsilon_{i} \pm 1,1 \leq i \leq m} \epsilon_{1} \ldots \epsilon_{m} P_{m}\left(\epsilon_{1} x_{1}+\ldots+\epsilon_{m} x_{m}\right) \\
& =\frac{1}{m ! 2^{m}} \sum_{\epsilon_{i} \pm 1,1 \leq i \leq m} \epsilon_{1} \ldots \epsilon_{m} A_{m}\left(\epsilon_{1} x_{1}+\ldots+\epsilon_{m} x_{m}\right)^{m} \\
& =A_{m}\left(x_{1}, \ldots, x_{m}\right)
\end{aligned}
$$

Para $k<m$,

$$
\begin{aligned}
\phi_{m}\left(P_{k}\right) & =\frac{1}{m ! 2^{m}} \sum_{\epsilon_{i} \pm 1,1 \leq i \leq m} \epsilon_{1} \ldots \epsilon_{m} P_{k}\left(\epsilon_{1} x_{1}+\ldots+\epsilon_{m} x_{m}\right) \\
& =\frac{1}{m ! 2^{m}} \sum_{\epsilon_{i} \pm 1,1 \leq i \leq m} \epsilon_{1} \ldots \epsilon_{m} A_{k}\left(\epsilon_{1} x_{1}+\ldots+\epsilon_{m} x_{m}\right)^{k} .
\end{aligned}
$$

Pela fórmula de Leibniz (2.2.7),

$$
\begin{aligned}
A_{k}\left(\epsilon_{1} x_{1}+\ldots+\epsilon_{m} x_{m}\right)^{k} & =\sum_{|\alpha|} \frac{k !}{n_{1} ! \ldots n_{m} !} A_{k}\left(\left(\epsilon_{1} x_{1}\right)^{n_{1}} \ldots\left(\epsilon_{m} x_{m}\right)^{n_{m}}\right) \\
& =\sum_{|\alpha|} \frac{k !}{n_{1} ! \ldots n_{m} !} \epsilon_{1}^{n_{1}} \ldots \epsilon_{m}^{n_{m}} A_{k}\left(x_{1}^{n_{1}} \ldots x_{m}^{n_{m}}\right)
\end{aligned}
$$


onde a soma é sobre todas as m-uplas $\alpha=\left(n_{1}, \ldots, n_{m}\right) \in \mathbb{N}^{m}$ satisfazendo $|\alpha|=n_{1}+\ldots+n_{m}=k, \log \mathrm{o}$

$$
\phi_{m}\left(P_{k}\right)=\frac{k !}{m ! 2^{m}} \sum_{|\alpha|} \frac{1}{n_{1} ! \ldots m_{m} !} \sum_{\epsilon_{i}= \pm 1,1 \leq i \leq m} \epsilon_{1}^{n_{1}+1} \ldots \epsilon_{m}^{n_{m}+1} A_{k}\left(x_{1}^{n_{1}} \ldots x_{m}^{n_{m}}\right) .
$$

Como $n_{1}+\ldots+n_{m}=k$, e $k<m$ então $n_{i}=0$ para algum $i=1, \ldots, m$, assim, $\sum_{\epsilon_{i}= \pm 1,1 \leq i \leq m} \epsilon_{1}^{n_{1}+1} \ldots \epsilon_{m}^{n_{m}+1}=0$. Portanto $\phi_{m}\left(P_{k}\right)=0$.

Seja $P: X \longrightarrow Y$ um polinômio de grau $\mathrm{m}$ tal que $P=P_{0}+P_{1}+\ldots+P_{m}$ então, pelo teorema acima, temos que $\phi_{m}(P)=\phi_{m}\left(P_{m}\right)$.

Se $x=x_{1}=\ldots,=x_{m}$ então

$$
P_{m}(x)=\frac{1}{m ! 2^{m}} \sum_{\epsilon_{i} \pm 1,1 \leq i \leq m} \epsilon_{1} \ldots \epsilon_{m} P\left(\left(\epsilon_{1}+\ldots+\epsilon_{m}\right) x\right) .
$$

\subsection{Polinômios contínuos}

Vamos denotar por $\mathcal{P}\left({ }^{m} X ; Y\right)$ o espaço vetorial de todos os polinômios m-homogêneos contínuos de $X$ em $Y$.

O próximo teorema nos dará uma relação entre os polinômios m-homogêneos contínuos e a as aplicações m-lineares contínuas simétricas.

Teorema 2.4.1. Sejam $X, Y$ espaços normados sobre o mesmo corpo $\mathbb{I K}$ e $m \in \mathbb{N}$. Para cada $P \in \mathcal{P}_{a}\left({ }^{m} X ; Y\right)$ seja $A \in \mathcal{L}_{a s}\left({ }^{m} X ; Y\right)$ tal que $P(x)=A x^{m}$ para cada $x \in X$. Então as seguintes afirmações são equivalentes:

1. $A \in \mathcal{L}_{s}\left({ }^{m} X ; Y\right)$.

2. $P \in \mathcal{P}\left({ }^{m} X ; Y\right)$.

3. P é contínuo na origem.

4. Existe $M>0$ tal que $\|P(x)\| \leq M\|x\|^{m}$ para cada $x \in X$.

Demonstração: $(1 \Rightarrow 2)$ Seja $x_{0} \in X$. Dado $\epsilon>0$, da continuidade de $A$ existe $\delta>0$ tal que, para qualquer $\left(x_{1}, \ldots, x_{m}\right) \in X^{m}$ com $\left\|\left(x_{1}, \ldots, x_{m}\right)-\left(x_{0}, \stackrel{(m)}{?}, x_{0}\right)\right\|<\delta$ temos que $\left\|A\left(x_{1}, \ldots, x_{m}\right)-A\left(x_{0}, \stackrel{(m)}{!}, x_{0}\right)\right\|<\epsilon$. Em particular, para $(x, \stackrel{(m)}{!}, x) \in X^{m}$ tal que $\left\|(x, \stackrel{(m)}{?}, x)-\left(x_{0}, \stackrel{(m)}{,}, x_{0}\right)\right\|<\delta$, ou seja, $\left\|x-x_{0}\right\|<\delta$, temos que $\left\|A x^{m}-A x_{0}^{m}\right\|<\epsilon$. 
Consequentemente, $\left\|P(x)-P\left(x_{0}\right)\right\|<\epsilon$ sempre que $\left\|x-x_{0}\right\|<\delta$. Portanto $P$ é contínua. ( $2 \Rightarrow 3)$ Trivial.

$(3 \Rightarrow 4)$ Seja $x \in X$. Como $P$ é contínuo na origem, dado $\epsilon=1$ existe $\delta>0$ tal que, para $x \in X \operatorname{com}\|x\|<\delta$ temos que $\|P(x)\| \leq 1$. Se $x \neq 0$, tomando $y=\frac{\delta x}{2\|x\|}$, temos que $\|y\|=\left\|\frac{\delta x}{2\|x\|}\right\|=\frac{\delta}{2}<\delta$. Logo, $\|P(y)\| \leq 1$. Agora como, $\|P(y)\|=\left\|A y^{m}\right\|=\frac{\delta^{m}}{2^{m}} \cdot \frac{\left\|A x^{m}\right\|}{\|x\|^{m}}$, temos que $\left\|A x^{m}\right\| \leq \frac{2^{m}}{\delta^{m}}\|x\|^{m}$. Considerando $M=\frac{2^{m}}{\delta^{m}}$, temos que $\|P(x)\| \leq M\|x\|^{m}$ para cada $x \in X$ e $x \neq 0$. Como para $x=0$ a desigualdade vale para todo $M>0$, segue o resultado.

(4 $\Rightarrow 1$ ) Seja $M>0$ tal que $\|P(x)\| \leq M\|x\|^{m}$ para cada $x \in X$. Vamos mostrar que $A$ é contínua. Sejam $x_{1}, \ldots, x_{m} \in X, \operatorname{com}\left\|x_{i}\right\| \leq \frac{1}{m}$ para cada $i=1, \ldots, m$, usando a fórmula da polarização (teorema 2.2.8), temos:

$$
\begin{aligned}
\left\|A\left(x_{1}, \ldots, x_{m}\right)\right\| & =\left\|\frac{1}{m ! 2^{m}} \sum_{\epsilon_{i} \pm 1,1 \leq i \leq m} \epsilon_{1} \ldots \epsilon_{m} A\left(\epsilon_{1} x_{1}+\ldots+\epsilon_{m} x_{m}\right)^{m}\right\| \\
& \leq \frac{1}{m ! 2^{m}} \sum_{\epsilon_{i} \pm 1,1 \leq i \leq m}\left|\epsilon_{1}\right| \ldots\left|\epsilon_{m}\right|\left\|A\left(\epsilon_{1} x_{1}+\ldots+\epsilon_{m} x_{m}\right)^{m}\right\| \\
& =\frac{1}{m ! 2^{m}} \sum_{\epsilon_{i} \pm 1,1 \leq i \leq m}\left\|P\left(\epsilon_{1} x_{1}+\ldots+\epsilon_{m} x_{m}\right)\right\| \\
& \leq \frac{1}{m ! 2^{m}} \sum_{\epsilon_{i} \pm 1,1 \leq i \leq m} M\left\|\epsilon_{1} x_{1}+\ldots+\epsilon_{m} x_{m}\right\|^{m} \\
& \leq \frac{1}{m ! 2^{m}} \sum_{\epsilon_{i} \pm 1,1 \leq i \leq m} M\left(\left|\epsilon_{1}\right|\left\|x_{1}\right\|+\ldots+\left|\epsilon_{m}\right|\left\|x_{m}\right\|\right)^{m} \\
& =\frac{1}{m ! 2^{m}} \sum_{\epsilon_{i} \pm 1,1 \leq i \leq m} M\left(\left\|x_{1}\right\|+\ldots+\left\|x_{m}\right\|\right)^{m} \cdot
\end{aligned}
$$

Como $\left\|x_{i}\right\| \leq \frac{1}{m}$ para cada $i=1, \ldots, m$ então $\sum_{i=1}^{m}\left\|x_{i}\right\| \leq 1$. Assim,

$$
\begin{gathered}
\frac{1}{m ! 2^{m}} \sum_{e_{i} \pm 1,1 \leq i \leq m} M\left(\left\|x_{1}\right\|+\ldots+\left\|x_{m}\right\|\right)^{m} \\
\leq \frac{1}{m ! 2^{m}} \sum_{e_{i} \pm 1,1 \leq i \leq m} M=\frac{M}{m !} .
\end{gathered}
$$

Agora para cada $x_{1}, \ldots, x_{m} \in X^{m}$, com $x_{i} \neq 0,1 \leq i \leq m$, considere $y_{i}=\frac{x_{i}}{m\left\|x_{i}\right\|}$, e assim 
$\left\|y_{i}\right\|=\left\|\frac{x_{i}}{m\left\|x_{i}\right\|}\right\|=\frac{1}{m}, \mathrm{e}$

$$
\left\|A\left(y_{1}, \ldots, y_{m}\right)\right\|=\frac{\left\|A\left(x_{1}, \ldots, x_{m}\right)\right\|}{m^{m}\left\|x_{1}\right\| \ldots\left\|x_{m}\right\|} \leq \frac{M}{m !},
$$

ou seja,

$$
\left\|A\left(x_{1}, \ldots, x_{m}\right)\right\| \leq \frac{M}{m !} m^{m}\left\|x_{1}\right\| \ldots\left\|x_{m}\right\| .
$$

Assim, para cada $x_{i} \in X \operatorname{com} i=1, \ldots, m$ temos que

$$
\left\|A\left(x_{1}, \ldots, x_{m}\right)\right\| \leq K\left\|x_{1}\right\| \ldots\left\|x_{m}\right\|,
$$

onde $K=\frac{M}{m !} m^{m}$. Portanto pelo teorema 2.1 .2 a aplicação $A$ é contínua.

A cada polinômio $P \in \mathcal{P}\left({ }^{m} X ; Y\right)$ associamos um número dado por

$\|P\|=\sup \{\|P(x)\|:\|x\|=1\}$. De maneira análoga a proposição 2.1.3 temos que a função

$$
\begin{aligned}
\|\cdot\|: \mathcal{P}\left({ }^{m} X ; Y\right) & \longrightarrow \mathbb{R}^{+} \\
P & \longmapsto\|P\| \text { é uma norma. }
\end{aligned}
$$

No que segue vamos estudar outras maneiras de caracterizar a $\|P\|$ e suas propriedades.

Proposição 2.4.2. Sejam $X, Y$ espaços normados sobre o mesmo corpo $\mathbb{K}, m \in \mathbb{N} e$ $P \in \mathcal{P}\left({ }^{m} X ; Y\right)$ então:

1. $\|P\|=\sup \{\|P(x)\|:\|x\| \leq 1\}$

$$
=\sup \left\{\frac{\|P(x)\|}{\|x\|^{m}}: x \neq 0\right\}=\inf \left\{M \geq 0:\|P(x)\| \leq M\|x\|^{m}\right\} \text {. }
$$

2. Para cada $x \in X$, temos que $\|P(x)\| \leq\|P\|\|x\|^{m}$.

Demonstração: (1) É imediato que

$$
\sup \{\|P(x)\|:\|x\|=1\} \leq \sup \{\|P(x)\|:\|x\| \leq 1\} .
$$

Vamos mostrar o outro lado da desigualdade. Seja $x \in B_{X}$. Se $x \neq 0$, tomando $y=\frac{x}{\|x\|} \mathrm{e}$ $A$ a aplicação m-linear associada a $P$, temos que

$$
\|P(y)\|=\left\|A y^{m}\right\|=\frac{\left\|A x^{m}\right\|}{\|x\|^{m}} \geq\left\|A x^{m}\right\|=\|P(x)\|,
$$

uma vez que $\|x\| \leq 1$. Logo $\sup \{\|P(w)\|:\|w\|=1\} \geq\|P(x)\|$ para cada $x \in B_{X}$. Se $x=0$, a desigualdade também vale. Assim, segue que

$$
\sup \{\|P(x)\|:\|x\|=1\} \geq \sup \{\|P(x)\|:\|x\| \leq 1\} .
$$


Portanto, temos que

$$
\sup \{\|P(x)\|:\|x\| \leq 1\}=\sup \{\|P(x)\|:\|x\|=1\} .
$$

Seja $M \geq 0$ tal que $\|P(x)\| \leq M\|x\|^{m}$ para todo $x \in X$. Se $\|x\|=1$ então $\|P(x)\| \leq M$. Assim $\sup \{\|P(x)\|:\|x\|=1\} \leq M$, e consequentemente

$$
\sup \{\|P(x)\|:\|x\|=1\} \leq \inf \left\{M \geq 0:\|P(x)\| \leq M\|x\|^{m}\right\} .
$$

Para verificar a outra desigualdade, seja $x \in X, x \neq 0$, e considere $y=\frac{x}{\|x\|}$, então temos que

$$
\|P(y)\|=\left\|P\left(\frac{x}{\|x\|}\right)\right\|=\frac{\|P(x)\|}{\|x\|^{m}} \leq \sup \{\|P(z)\|:\|z\|=1\} .
$$

Logo, $\|P(x)\| \leq \sup \{\|P(z)\|:\|z\|=1\}\|x\|^{m}$. Como a desigualdade também vale quando $x=0$, concluímos que

$$
\inf \left\{M \geq 0:\|P(x)\| \leq M\|x\|^{m}\right\} \leq \sup \{\|P(z)\|:\|z\|=1\} .
$$

Portanto temos a igualdade

$$
\sup \{\|P(x)\|:\|x\|=1\}=\inf \left\{M \geq 0:\|P(x)\| \leq M\|x\|^{m}\right\} .
$$

Seja $x \in X \operatorname{com}\|x\| \leq 1$. Se $x \neq 0$, então

$$
\|P(x)\| \leq \frac{\|P(x)\|}{\|x\|^{m}} \leq \sup \left\{\frac{\|P(x)\|}{\|x\|^{m}}: x \neq 0\right\},
$$

logo $\sup \{\|P(x)\|:\|x\| \leq 1\} \leq \sup \left\{\frac{\|P(x)\|}{\|x\|^{m}}: x \neq 0\right\}$. Para termos o outro lado da desigualdade, dado $x \neq 0$, tomando $y=\frac{x}{\|x\|}$, temos que

$\|P(y)\|=\left\|P\left(\frac{x}{\|x\|}\right)\right\| \leq \sup \{\|P(z)\|:\|z\| \leq 1\}$. Se $\|x\| \geq 1$ então $\frac{\|P(x)\|}{\|x\|^{m}} \leq \frac{\|P(x)\|}{\|x\|} \leq \sup \{\|P(z)\|:\|z\| \leq 1\}$. Logo, $\sup \left\{\frac{\|P(x)\|}{\|x\|^{m}}: x \neq 0\right\} \leq \sup \{\|P(z)\|:\|z\| \leq 1\}$. Portanto, temos a igualdade

$$
\sup \left\{\frac{\|P(x)\|}{\|x\|^{m}}: x \neq 0\right\}=\sup \{\|P(x)\|:\|x\| \leq 1\} .
$$

(2) Seja $x \in X$. Se $x=0$, vale a igualdade. Se $x \neq 0$ então

$$
\frac{\|P(x)\|}{\|x\|^{m}} \leq \sup \left\{\frac{\|P(x)\|}{\|x\|^{m}}: x \neq 0\right\}=\|P\| .
$$

Logo $\|P(x)\| \leq\|P\|\|x\|^{m}$.

O próximo teorema foi provado por Martin. Ele nos dá uma relação entre $\|\hat{A}\|$ e $\|A\|$. 
Teorema 2.4.3. (Martin - 1932)

Sejam $X, Y$ espaços normados e $m \in \mathbb{N}$. Então $\mathcal{L}_{s}\left({ }^{m} X ; Y\right)$ e $\mathcal{P}\left({ }^{m} X ; Y\right)$ são topologicamente isomorfos $e$

$$
\|\hat{A}\| \leq\|A\| \leq \frac{m^{m}}{m !}\|\hat{A}\| \text {. }
$$

Demonstração: Seja $\phi$ a aplicação dada no teorema 2.3.4, então tomando $\psi=\left.\phi\right|_{\mathcal{L}_{s}\left({ }^{m} X ; Y\right)}$ temos que $\psi$ é um isomorfismo. Vamos provar a seguinte desigualdade

$$
\|\hat{A}\| \leq\|A\| \leq \frac{m^{m}}{m !}\|\hat{A}\|
$$

para cada $A \in \mathcal{L}_{s}\left({ }^{m} X ; Y\right)$. Se $m=0$, e $m=1$, temos que $\psi(A)=A$, logo temos que

$$
\|\hat{A}\|=\|\psi(A)\|=\|A\| \leq \frac{m^{m}}{m !}\|\hat{A}\| .
$$

Seja $m>1$, então

$$
\|\hat{A}(x)\|=\|\psi(A)(x)\|=\|P(x)\|=\left\|A x^{m}\right\| \leq\|A\|\|x\|^{m},
$$

concluímos que

$$
\|\hat{A}\|=\sup \{\|\hat{A}(x)\|:\|x\|=1\} \leq \sup \left\{\|A\|\|x\|^{m}:\|x\|=1\right\}=\|A\| .
$$

Resta mostrar o outro lado da desigualdade.

Seja $\left(x_{1}, \ldots, x_{m}\right) \in X^{m}$, pela fórmula da polarização (2.2.8), temos que

$$
\begin{aligned}
\left\|A\left(x_{1}, \ldots, x_{m}\right)\right\| & \leq \frac{1}{m ! 2^{m}} \sum_{\epsilon_{i}= \pm 1,1 \leq i \leq m}\left\|A\left(\epsilon_{1} x_{1}+\ldots+\epsilon_{m} x_{m}\right)^{m}\right\| \\
& =\frac{1}{m ! 2^{m}} \sum_{\epsilon_{i}= \pm 1,1 \leq i \leq m}\left\|P\left(\epsilon_{1} x_{1}+\ldots+\epsilon_{m} x_{m}\right)\right\| \\
& =\frac{1}{m ! 2^{m}} \sum_{\epsilon_{i}= \pm 1,1 \leq i \leq m}\left\|\psi(A)\left(\epsilon_{1} x_{1}+\ldots+\epsilon_{m} x_{m}\right)\right\| \\
& \leq \frac{1}{m ! 2^{m}} \sum_{\epsilon_{i}= \pm 1,1 \leq i \leq m}\|\psi(A)\| \cdot\left\|\left(\epsilon_{1} x_{1}+\ldots+\epsilon_{m} x_{m}\right)\right\|^{m}
\end{aligned}
$$

pelo teorema 2.4 .2 , pois $\psi(A) \in \mathcal{P}\left({ }^{m} X ; Y\right)$, logo

$$
\begin{aligned}
\left\|A\left(x_{1}, \ldots, x_{m}\right)\right\| & \leq \frac{1}{m ! 2^{m}} \sum_{\epsilon_{i}= \pm 1,1 \leq i \leq m}\|\psi(A)\|\left(\left\|x_{1}\right\|+\ldots+\left\|x_{m}\right\|\right)^{m} \\
& =\frac{1}{m ! 2^{m}} \cdot 2^{m}\|\psi(A)\|\left(\left\|x_{1}\right\|+\ldots+\left\|x_{m}\right\|\right)^{m} .
\end{aligned}
$$


Então, se para cada $i=1, \ldots, m,\left\|x_{i}\right\|=1$, temos que:

$$
\|A\| \leq \frac{1}{m !}\|\psi(A)\|(1+\stackrel{(m)}{\cdot}+1)^{m}=\frac{1}{m !}\|\psi(A)\| m^{m} .
$$

Portanto $\|A\| \leq \frac{m^{m}}{m !}\|\hat{A}\|$.

Corolário 2.4.4. Sejam $X$ um espaço nomado e $Y$ um espaço de Banach sobre o corpo $\mathbb{K}$ e $m \in \mathbb{N}$. Então $\mathcal{P}\left({ }^{m} X ; Y\right)$ é um espaço de Banach.

Demonstração: Segue da proposição 2.2.3 e do teorema 2.4.3.

A constante $\frac{m^{m}}{m !}$ é a melhor possível, como mostra o seguinte exemplo dado por Nachbin.

Exemplo 2.4.5. Sejam $l_{1}$ e $x=\left(x_{n}\right)_{n} \in l_{1}$. Seja $P: l_{1} \longrightarrow \mathbb{K}$ dado por $P(x)=x_{1} \cdot x_{2} \cdot \ldots \cdot x_{m}$. Pelo exemplo 2.1.6 temos que $P \in \mathcal{P}\left({ }^{m} l_{1}\right)$. Se $\left(e_{i}\right)_{i}$ é a base canônica de $l_{1}$ então

$$
A\left(e_{1}, \ldots, e_{m}\right)=\frac{1}{m ! 2^{m}} \sum_{\epsilon_{i}= \pm 1,1 \leq i \leq m} \epsilon_{1} \ldots \epsilon_{m} P\left(\sum_{i=1}^{m} \epsilon_{i} e_{i}\right)=\frac{1}{m !} .
$$

Assim $\|A\| \geq \frac{1}{m !}$. Por outro lado, se $\|x\|=\sum_{i=1}^{\infty}\left|x_{i}\right| \leq 1$, e como

$$
\left|x_{1}\right| \ldots\left|x_{m}\right| \leq \frac{1}{m^{m}}\left(\sum_{i=1}^{m}\left|x_{i}\right|\right)^{m}
$$

temos que $|\hat{A}(x)| \leq \frac{1}{m^{m}}$. Se $x=\left(\frac{1}{m}, \stackrel{(m)}{.}, \frac{1}{m}, 0, \ldots\right)$ então $\hat{A}(x)=\frac{1}{m^{m}}$. Logo $\|\hat{A}\|=\frac{1}{m^{m}}, e$ assim $\|A\|=\frac{m^{m}}{m !}\|\hat{A}\|$.

Teorema 2.4.6. Sejam $X, Y, Z$ espaços normados sobre o corpo $\mathbb{K}, m \in \mathbb{N}, P: X \longrightarrow Y$ um polinômio m-homogêneo contínuo, $T: Z \longrightarrow X$ e $S: Y \longrightarrow Z$ aplicaçôes lineares contínuas. Então $P \circ T \in \mathcal{P}\left({ }^{m} Z ; Y\right)$ e $S \circ P \in \mathcal{P}\left({ }^{m} X ; Z\right)$.

Demonstração: Como $P$ é um polinômio m-homogêneo, existe $B: X^{m} \longrightarrow Y$ m-linear tal que $P(x)=B x^{m}$ para cada $x \in X$.

Seja $C_{1}: Z^{m} \longrightarrow Y$ dado por:

$$
C_{1}\left(z_{1}, \ldots, z_{m}\right)=B\left(T\left(z_{1}\right), \ldots, T\left(z_{m}\right)\right)
$$


para cada $\left(z_{1}, \ldots, z_{m}\right) \in Z^{m}$, então $C_{1}$ é m-linear e $(P \circ T)(z)=C_{1} z^{m}$ para cada $z^{*} \in Z$. Logo $P \circ T \in \mathcal{P}\left({ }^{m} Z ; Y\right)$.

Seja $C_{2}: X^{m} \longrightarrow Z$ dado por:

$$
C_{2}\left(x_{1}, \ldots, x_{m}\right)=S\left(B\left(x_{1}, \ldots, x_{m}\right)\right)
$$

para cada $\left(x_{1}, \ldots, x_{m}\right) \in X^{m}$. Então $C_{2}$ é m-linear e $(S \circ P)(x)=C_{2} x^{m}$ para cada $x \in X$. Logo $S \circ P \in \mathcal{P}\left({ }^{m} X ; Z\right)$.

Sejam $X, Y$ espaços normados sobre o corpo $\mathbb{I K}$. Vamos denotar por $\mathcal{P}(X ; Y)$ o espaço vetorial de todos os polinômios contínuos de $X$ em $Y$. Se $Y=\mathbb{K}$, então denotamos $\mathcal{P}(X ; Y)=\mathcal{P}(X)$.

O próximo teorema nos dará critérios para a continuidade do polinômio $P$.

Teorema 2.4.7. Sejam $X, Y$ espaços normados sobre o mesmo corpo $\mathbb{K}$ e $m \in \mathbb{N}$. Seja $P: X \longrightarrow Y$ um polinômio de grau $m$ dado por $P=P_{0}+\ldots+P_{m}$. Então são equivalentes:

1. $P_{0}, \ldots, P_{m}$ são contínuos.

2. Pé contínuo.

3. P é contínuo na origem.

4. P é limitado em $B_{X}$.

Demonstração: $(1 \Rightarrow 2 \Rightarrow 3)$ é imediato.

$(2 \Rightarrow 1)$ Suponhamos que $\mathrm{P}$ é contínuo. Vamos mostrar que $P_{j}$ é contínuo para cada $j=0, \ldots, m$. A prova será feita por indução sobre $m$. Para $m=0$ é evidente. Vamos supor que vale para $m-1$. Vamos mostrar que é válido para $m$. Para todo $x \in X \mathrm{e}$ $\alpha \in \mathbb{K}$, temos que

$$
\text { (1) } \sum_{j=0}^{m} P_{j}(x)=P(x) \quad \text { e } \quad \text { (2) } \sum_{j=0}^{m} P_{j}(\alpha x)=P(\alpha x) \text {. }
$$

Multiplicando (1) por $\alpha^{m} \in \mathbb{K}$ e subtraindo (2) temos que

$$
\alpha^{m} P(x)-P(\alpha x)=\sum_{j=0}^{m-1}\left(\alpha^{m}-\alpha^{j}\right) P_{j}(x)
$$

para quaisquer $x \in X$ e $\alpha \in \mathbb{K}$.

Como $P$ é contínuo, temos que $\alpha^{m} P(x)-P(\alpha x)$ é contínuo para cada $x \in X$. Assim, 
se $\alpha \in \mathbb{K}$ é tal que $\alpha^{m}-\alpha^{j} \neq 0$ para cada $j=0, \ldots, m-1$, temos que $\sum_{j=0}^{m-1} Q_{j}(x)$ é um polinômio contínuo, onde $Q_{j}=\left(\alpha^{m}-\alpha^{j}\right) P_{j} \in \mathcal{P}_{a}\left({ }^{j} X ; Y\right)$ para cada $j=0, \ldots, m-1$. Da hipótese de indução, segue que $Q_{j}$ é contínuo para todo $j=0, \ldots, m-1$. Assim $P_{0}, \ldots, P_{m-1}$ são contínuos, e como $P$ também é contínuo, segue que $P-\sum_{j=0}^{m-1} P_{j}=P_{m}$ é contínuo.

( $3 \Rightarrow 4)$ Se $P$ é contínuo na origem, então, pela equivalência $1 \Longleftrightarrow 2$, temos que $P_{j}$ é contínuo na origem para cada $j=0, \ldots, m$. Assim segue do teorema 2.4.1 que para cada $j=0, \ldots, m$, existe $M_{j}>0$ tal que $\left\|P_{j}(x)\right\| \leq M_{j}$ para qualquer $x \in X$ com $\|x\| \leq 1$. Como $\|P(x)\| \leq \sum_{j=0}^{m}\left\|P_{j}(x)\right\|$, temos que

$$
\|P(x)\| \leq \sum_{j}^{m} M_{j}
$$

para qualquer $x \in B_{X}$. Portanto $P$ é limitado na bola unitária fechada.

$(4 \Rightarrow 1)$ Seja $P=P_{0}+\ldots+P_{m} \in \mathcal{P}_{a}\left({ }^{j} X ; Y\right)$ limitado na bola unitária fechada. Como $P_{0}$ é contínuo, podemos considerar $P_{0}=0$, isto é, $P=P_{1}+\ldots+P_{m}$. Então pela polarização de $P$ com respeito a quaisquer $x_{1}, \ldots, x_{m} \in X$ (teorema 2.3.8) segue que

$$
\phi_{m}(P)=\phi_{m}\left(P_{0}\right)+\phi_{m}\left(P_{1}\right)+\ldots+\phi_{m}\left(P_{m}\right)=A_{m}\left(x_{1}, \ldots, x_{m}\right),
$$

pois $\phi_{m}\left(P_{i}\right)=0$ para cada $i<m$. Como $P$ é limitado em $B_{X}$, existe $M>0$ tal que $\|P(x)\| \leq M$ para qualquer $x \in B_{X}$. Assim para $x_{1}, \ldots, x_{m} \in X$ com $\left\|x_{1}\right\|+\ldots+\left\|x_{m}\right\| \leq 1$, temos que

$$
\begin{aligned}
\left\|A_{m}\left(x_{1}, \ldots, x_{m}\right)\right\| & =\left\|\phi_{m}(P)\right\| \\
& =\left\|\frac{1}{m ! 2^{m}} \sum_{\epsilon_{i}= \pm 1,1 \leq i \leq m} \epsilon_{1} \ldots \epsilon_{m} P\left(\epsilon_{1} x_{1}+\ldots+\epsilon_{m} x_{m}\right)\right\| \\
& \leq \frac{1}{m ! 2^{m}}\left\|\sum_{\epsilon_{i}= \pm 1,1 \leq i \leq m} P\left(\epsilon_{1} x_{1}+\ldots+\epsilon_{m} x_{m}\right)\right\| \leq \frac{M}{m !}
\end{aligned}
$$

pois $\left\|\left(\epsilon_{1} x_{1}+\ldots+\epsilon_{m} x_{m}\right)\right\| \leq 1$. Logo $\left\|A_{m}\left(x_{1}, \ldots, x_{m}\right)\right\| \leq \frac{M}{m !}$ se $\left\|x_{1}\right\|+\ldots+\left\|x_{m}\right\| \leq 1$. Agora, vamos considerar qualquer $x=\left(x_{1}, \ldots, x_{m}\right) \in X^{m}$ tal que $x_{i} \neq 0$ para todo $i=1, \ldots, m$. Vamos definir $y_{i}=\frac{x_{i}}{m\left\|x_{i}\right\|}$ para cada $i=1, \ldots, m$. Então $\left\|y_{1}\right\|+\ldots+\left\|y_{m}\right\| \leq 1$ e pelo resultado provado acima, obtemos que

$$
\left\|A_{m}\left(y_{1}, \ldots, y_{m}\right)\right\|=\frac{1}{m^{m}\left\|x_{1}\right\| \ldots\left\|x_{m}\right\|}\left\|A_{m}\left(x_{1}, \ldots, x_{m}\right)\right\| \leq \frac{M}{m !} .
$$


Portanto

$$
\left\|A_{m}\left(x_{1}, \ldots, x_{m}\right)\right\| \leq \frac{m^{m} M}{m !}\left\|x_{1}\right\| \ldots\left\|x_{m}\right\|
$$

para qualquer $x=\left(x_{1}, \ldots, x_{m}\right) \in X^{m}$. Então se $K=\frac{m^{m} M}{m}$, temos que

$\left\|P_{m}(x)\right\| \leq K\|x\|^{m}$ para qualquer $x \in X$. Assim, segue do teorema 2.4.1 que $P_{m}$ é contínuo e $P-P_{m}$ é limitado na bola unitária fechada. Repetindo o mesmo raciocínio para $P_{1}, \ldots, P_{m-1}$, podemos concluir que $P_{m-1}, \ldots, P_{1}$ são contínuos.

O próximo teorema foi obtido por Mazur e Orlicz em 1934. Ele é do tipo BanachSteinhaus para polinômios homogêneos contínuos.

\section{Teorema 2.4.8. ( Banach-Steinhaus para polinômios)}

Sejam $X, Y$ espaços de Banach e $\left(P_{n}\right)_{n}$ uma sequência em $\mathcal{P}\left({ }^{m} X ; Y\right)$. Se $P$ é o limite pontual da sequência $\left(P_{n}\right)_{n}$ então $P \in \mathcal{P}\left({ }^{m} X ; Y\right)$.

Demonstração: Seja $\left(P_{n}\right)_{n} \subset \mathcal{P}\left({ }^{m} X ; Y\right)$ uma sequência que converge pontualmente. Para cada $n \in \mathbb{N}$ seja $A_{n} \in \mathcal{L}_{s}\left({ }^{m} X ; Y\right)$ a aplicação m-linear simétrica associada a $P_{n} \in \mathcal{P}\left({ }^{m} X ; Y\right)$. Se $P$ é o limite pontual da sequência $\left(P_{n}\right)_{n}$, então pela fórmula da polarização (teorema 2.2.8),

$$
\begin{gathered}
\lim _{n \rightarrow \infty} A_{n}\left(x_{1}, \ldots, x_{m}\right)=\lim _{n \rightarrow \infty} \frac{1}{m ! 2^{m}} \sum_{\epsilon= \pm 1 ; 1 \leq i \leq m} \epsilon_{1} \ldots \epsilon_{m} A_{n}\left(\epsilon_{1} x_{1}+\ldots+\epsilon_{m} x_{m}\right)^{m} \\
=\frac{1}{m ! 2^{m}} \sum_{\epsilon= \pm 1 ; 1 \leq i \leq m} \epsilon_{1} \ldots \epsilon_{m} \lim _{n \rightarrow \infty} P_{n}\left(\epsilon_{1} x_{1}+\ldots+\epsilon_{m} x_{m}\right) \\
=\frac{1}{m ! 2^{m}} \sum_{\epsilon= \pm 1 ; 1 \leq i \leq m} \epsilon_{1} \ldots \epsilon_{m} P\left(\epsilon_{1} x_{1}+\ldots+\epsilon_{m} x_{m}\right)
\end{gathered}
$$

para qualquer $\left(x_{1}, \ldots, x_{m}\right) \in X^{m}$. Consequentemente, para qualquer $\left(x_{1}, \ldots, x_{m}\right) \in X^{m}$ a sequência $\left(A_{n}\left(x_{1}, \ldots, x_{m}\right)\right)_{n}$ é convergente. Consideremos $A$ o limite pontual da sequência $\left(A_{n}\right)_{n}$. Então claramente $A \in \mathcal{L}_{a s}\left({ }^{m} X ; Y\right)$. Como $\mathcal{L}\left({ }^{m} X ; Y\right)$ é isomorfo a $\mathcal{L}\left(X ; \mathcal{L}\left({ }^{m-1} X ; Y\right)\right)$, a sequência $\left(A_{n}\right)_{n}$ pode ser vista como uma sequência em $\mathcal{L}\left(X ; \mathcal{L}\left({ }^{m-1} X ; Y\right)\right)$. Assim, pelo teorema de Banach-Steinhaus para aplicações lineares ( teorema 1.2.10), temos que $A \in \mathcal{L}\left(X ; \mathcal{L}\left({ }^{m-1} X ; Y\right)\right.$ ). Como para qualquer $x \in X$

$$
P(x)=\lim _{n \rightarrow \infty} P_{n}(x)=\lim _{n \rightarrow \infty} A_{n}(x, \stackrel{(n)}{.}, x)=A(x, \stackrel{(n)}{.}, x),
$$

concluímos que $P \in \mathcal{P}\left({ }^{m} X ; Y\right)$. 
Exemplo 2.4.9. Seja $P: l_{p} \longrightarrow \mathbb{K}$ dado por:

$$
P(x)=\sum_{j=1}^{\infty} x_{j}^{2} \quad \text { com } x=\left(x_{j}\right)_{j} \in l_{p} .
$$

Afirmamos que $P \in \mathcal{P}\left({ }^{2} l_{p}\right)$.

De fato: Para cada $n \in \mathbb{N}$ definamos $P_{n}: l_{p} \longrightarrow \mathbb{K}$ por:

$$
P_{n}(x)=\sum_{j=1}^{n} x_{j}^{2} \quad \operatorname{com} x=\left(x_{j}\right)_{j} \in l_{p}
$$

Sabemos do exemplo 2.3.2 que $P_{n} \in \mathcal{P}\left({ }^{2} l_{p}\right)$, e para cada $x=\left(x_{j}\right)_{j} \in l_{p}$,

$$
P_{n}(x) \stackrel{n \rightarrow \infty}{\longrightarrow} P(x)
$$

logo, pelo teorema acima $P \in \mathcal{P}\left({ }^{2} l_{p}\right)$.

Teorema 2.4.10. Sejam $X, Y$ espaços normados sobre o corpo $\mathbb{K}, m \in \mathbb{N} e$ $P \in \mathcal{P}\left({ }^{m} X ; Y\right)$. Então $P$ é uniformemente contínuo sobre os limitados.

Demonstração: Sejam $P: X \longrightarrow Y$ um polinômio m-homogêneo e $A \in \mathcal{L}\left({ }^{m} X ; Y\right)$ a aplicação m-linear associada a $P$. Sejam $W \subset X$ limitado e $r>0$ tal que $W \subset B[0 ; r]$. Para cada $x, z \in W$ temos que:

$$
\begin{aligned}
& \|P(x)-P(z)\|=\|A(x, \stackrel{(m)}{!}, x)-A(z, \stackrel{(m)}{\cdot}, z)\| \\
& =\| A(x, \stackrel{(m)}{*}, x)-A\left(z, x,{ }^{(m-1)}, x\right)+A\left(z, x,{ }^{(m-1)}, x\right)-A\left(z, z, x,{ }^{(m-2)}, x\right) \\
& -A(z, z, x, \stackrel{(m-2)}{\cdots}, x)-\ldots-A(z, \stackrel{(m-1)}{\cdots}, z, x)+A(z, \stackrel{(m-1)}{\cdots}, z, x)-A(z, \stackrel{(m)}{\cdot}, z) \| \\
& \leq\left\|A\left(x-z,{ }^{(m-1)}, x\right)\right\|+\left\|A\left(z, x-z, x,{ }^{(m-2)}, x\right)\right\|+\ldots \\
& +\left\|A\left(z,{ }^{(m-1)}, z, x-z\right)\right\| \leq m\|A\| r^{m-1}\|x-z\| \text {. }
\end{aligned}
$$

Tomando $M=m\|A\| r^{m-1}$ temos que $\|P(x)-P(z)\| \leq M\|x-z\|$. Seja $\epsilon>0$, para $\delta=\frac{\epsilon}{M}$, temos que $\|P(x)-P(z)\|<\epsilon$ sempre que $x, y \in W$ e $\|x-z\|<\delta$. Portanto $P$ é uniformemente contínuo em cada conjunto limitado de $X$.

Corolário 2.4.11. Sejam $X, Y$ espaços de Banach e $P \in \mathcal{P}(X ; Y)$. Então $P$ é uniformemente contínuo sobre os limitados. 
Demonstração: Para cada $P \in \mathcal{P}(X ; Y)$, existem $m \in \mathbb{N}$ e $P_{k} \in \mathcal{P}\left({ }^{k} X ; Y\right)$, $k=0,1, \ldots, m$, tais que $P=P_{0}+P_{1}+\ldots+P_{m}$. Para cada $k=0, \ldots, m$ temos que $P_{k}$ é uniformemente contínuo sobre os limitados (pelo teorema acima). Então dado $\epsilon>0$ e $W \subset X$ limitado existe $\delta_{k}>0$ tais que se $x, y \in W$ e $\|x-y\|<\delta_{k}$ temos que $\left|P_{k}(x)-P_{k}(y)\right|<\frac{\epsilon}{m}$. Agora, tomando $\delta=\min \left\{\delta_{1}, \ldots, \delta_{m}\right\}$ e considerando que $x, y \in W$ tais que $\|x-y\|<\delta$, então

$$
\begin{gathered}
|P(x)-P(y)| \leq\left|P_{0}(x)-P_{0}(y)\right|+\left|P_{1}(x)-P_{1}(y)\right|+\ldots+\left|P_{m}(x)-P_{m}(y)\right| \\
<\frac{\epsilon}{m}+\stackrel{(m)}{\cdot}+\frac{\epsilon}{m}=\epsilon .
\end{gathered}
$$

Portanto $P$ é uniformemente contínuo sobre os limitados.

\subsection{Polinômios de tipo finito, fracamente contínuos e compactos}

Nesta seção vamos estudar alguns tipos de polinômios, os quais serão utilizados nos capítulos 3 e 4 .

Definição 2.5.1. Sejam $X, Y$ espaços de Banach e $m \in \mathbb{N}$. Dizemos que um polinômio $m$-homogêneo $P: X \longrightarrow Y$ é fracamente compacto (respectivamente, compacto) se leva conjuntos limitados de $X$ em conjuntos relativamente fracamente compactos (respectivamente, relativamente compactos) de $Y$.

Exemplos 2.5.2. 1. As aplicações lineares compactas dadas no capítulo 1, pg 13 são polinômios 1-homogêneos compactos.

2. Sejam $k \in \mathbb{N}, X, Y$ espaços de Banach com $Y$ reflexivo, cada polinômio k-homogêneo contínuo $P: X \longrightarrow Y$ é fracamente compacto.

De fato: Pelo teorema 2.4.7 temos que para cada $W \subset X$ limitado, $P(W)$ é limitado. Como $Y$ é reflexivo, pelo teorema de Eberlein-Smulian (1.2.24) $P(W)$ é relativamente fracamente compacto.

Se $X=Y=l_{2}$ o polinômio $P: l_{2} \longrightarrow l_{2}$ definido por

$$
P\left(\left(x_{n}\right)_{n}\right)=\left(\frac{1}{n} x_{n}^{k}\right)_{n}
$$

para todo $\left(x_{n}\right)_{n} \in l_{2}$, é fracamente compacto, pois $l_{2}$ é um espaço reflexivo. 
3. Para cada $k \geq 2$ definimos $Q: l_{2} \longrightarrow l_{1}$ por $Q\left(\left(x_{n}\right)_{n}\right)=\left(x_{n}^{k}\right)_{n}$ para todo $\left(x_{n}\right)_{n} \in l_{2}$. $O$ polinômio assim definido não é fracamente compacto. (ver por exemplo Hissadomi, [21], pg 54).

Proposição 2.5.3. Sejam $X, Y$ espaços de Banach, $m \in \mathbb{N}$ e $P \in \mathcal{P}_{a}\left({ }^{m} X ; Y\right)$. $P$ é fracamente compacto (respectivamente, compacto) se e somente se $P$ leva $B_{X}$ em um conjunto relativamente fracamente compacto (respectivamente, relativamente compacto) de $Y$.

Demonstração: $(\Rightarrow)$ Trivial, por definição.

$(\Leftarrow)$ Seja $W \subset X$ limitado, então existe $r>0$ tal que $W \subset B[0, r]$ em $X$. Logo

$P(W) \subset P(B[0, r])$. Mas, $P(B[0, r])=r^{m} P\left(B_{X}\right)$ e consequentemente

$\overline{P(W)}^{\omega} \subset r^{m}{\overline{P\left(B_{X}\right)}}^{\omega}$. Como por hipótese $P\left(B_{X}\right)$ é relativamente fracamente compac-

to em $Y$, segue que $r^{m} P\left(B_{X}\right)$ é relativamente fracamente compacto, e como

$\overline{P(W)}^{\omega} \subset r^{m}{\overline{P\left(B_{X}\right)}}^{\omega}$ temos que $\overline{P(W)}^{\omega}$ é fracamente compacto.

Definições 2.5.4. Sejam $X, Y$ espaços de Banach, $m \in \mathbb{N}$ e $P \in \mathcal{P}\left({ }^{m} X ; Y\right)$.

1. Dizemos que $P$ é fracamente contínuo sobre os limitados se para cada $W \subset X$ limitado, cada $x \in W$ e $\epsilon>0$ existem $\phi_{1}, \ldots, \phi_{n} \in X^{*}$ e $\delta>0$ tais que $\|P(x)-P(y)\|<\epsilon$ sempre que $\left|\phi_{i}(x-y)\right|<\delta$ com $y \in W$ e $i=1, \ldots, n$.

Vamos denotar por $\mathcal{P}_{w}\left({ }^{m} X ; Y\right)$ o espaço vetorial de todos os polinômios m-homogêneos contínuos de $X$ em $Y$ tais que restritos aos conjuntos limitados são fracamente contínuos.

2. Dizemos que $P$ é fracamente uniformemente contínuo sobre os limitados se para cada $W \subset X$ limitado e para cada $\epsilon>0$ existem $\phi_{1}, \ldots, \phi_{n} \in X^{*}$ e $\delta>0$ tais que $\|P(x)-P(y)\|<\epsilon$ sempre que $\left|\phi_{i}(x-y)\right|<\delta$ com $x, y \in W$ e $i=1, \ldots, n$.

Vamos denotar por $\mathcal{P}_{w u}\left({ }^{m} X ; Y\right)$ o espaço vetorial de todos os polinômios m-homogêneos contínuos de $X \mathrm{em} Y$ tais que restritos aos conjuntos limitados são fracamente uniformemente continuos.

3. Dizemos que $P$ é fracamente sequencialmente contínuo em $X$ se para cada $x \in X$ e cada sequência $\left(x_{n}\right)_{n}$ de $X$ tal que $x_{n} \stackrel{\omega}{\rightarrow} x$ temos que $P\left(x_{n}\right) \rightarrow P(x)$.

Vamos denotar por $\mathcal{P}_{\text {wsc }}\left({ }^{m} X ; Y\right)$ o espaço vetorial de todos os polinômios m-homogêneos de $X$ em $Y$ que são fracamente sequencialmente contínuos em cada $x \in X$. 
Proposição 2.5.5. Sejam $X, Y$ espaços de Banach e $m \in \mathbb{N}$. Então $P \in \mathcal{P}_{w}\left({ }^{m} X ; Y\right)$ se e somente se $\left.P\right|_{B_{X}}: B_{X} \longrightarrow Y$ é fracamente contínuo.

Demonstração: $(\Rightarrow)$ Trivial, por definição.

$(\Leftarrow)$ Seja $W \subset X$ limitado, então existe $r>0$ tal que $W \subset B[0, r]$ em $X$. Logo $P(W) \subset P(B[0, r])$. Mas $P(B[0, r])=r^{m} P\left(B_{X}\right)$. Seja $x \in W$ e $\epsilon>0$, como $\frac{x}{r} \in B_{X}$ e $\left.P\right|_{B_{X}}: B_{X} \longrightarrow Y$ é fracamente contínuo existem $\phi_{1}, \ldots, \phi_{n} \in X^{*}$ e $\delta>0$ tal que $\left\|P\left(\frac{x}{r}\right)-P(y)\right\|<\epsilon \cdot r^{m}$ sempre que $\left|\phi_{i}\left(\frac{x}{r}-y\right)\right|<\delta$ com $y \in B_{X}$ e $i=1, \ldots, n$. Tomando $\delta^{\prime}=r \delta$ temos que se $\left|\phi_{i}(x-z)\right|<\delta^{\prime} \operatorname{com} x, z \in W$, para cada $i=1, \ldots, n$. Logo $\frac{1}{r}\left|\phi_{i}(x-z)\right|<\frac{1}{r} \delta^{\prime}$, ou seja, $\left|\phi_{i}\left(\frac{x}{r}-\frac{z}{r}\right)\right|<\delta$, onde $\frac{x}{r}, \frac{z}{r} \in B_{X}$, assim, por hipótese, $\left\|P\left(\frac{x}{r}\right)-P\left(\frac{z}{r}\right)\right\|<r^{m} \epsilon$. Mas $\left\|P\left(\frac{x}{r}\right)-P\left(\frac{z}{r}\right)\right\|=\frac{1}{r^{m}}\|P(x)-P(z)\|$. Assim $\|P(x)-P(z)\|<\epsilon$ sempre que $\left|\phi_{i}(x-z)\right|<\delta^{\prime}$ para $i=1, \ldots, n$ com $x, z \in W$. Portanto $P \in \mathcal{P}_{w}\left({ }^{m} X ; Y\right)$.

Proposição 2.5.6. Sejam $X, Y$ espaços de Banach e $m \in \mathbb{N}$. Então $P \in \mathcal{P}_{w u}\left({ }^{m} X ; Y\right)$ se e somente se $\left.P\right|_{B_{X}}: B_{X} \longrightarrow Y$ é fracamente uniformemente contínuo.

Demonstração: Analogamente a proposição anterior.

A seguir, o nosso objetivo é estudar o fato de que o espaço $\mathcal{P}_{w}\left({ }^{m} X ; Y\right)$ coincide com o espaço $\mathcal{P}_{w u}\left({ }^{m} X ; Y\right), m \geq 1$. Para isso, vamos trabalhar com a aplicação linear associada ao polinômio $P$. Tal aplicação será também importante no estudo de alguns resultados apresentados no capítulo 4.

Proposição 2.5.7. Sejam $X, Y$ espaços de Banach, $m \in \mathbb{N}$ e $P \in \mathcal{P}\left({ }^{m} X ; Y\right)$. Seja $T_{P}: X \longrightarrow \mathcal{L}_{s}\left({ }^{m-1} X ; Y\right)$ dada por:

$$
T_{P}(x)\left(x_{2}, \ldots, x_{m}\right)=A\left(x, x_{2}, \ldots, x_{m}\right),
$$

onde $A$ é a aplicação m-linear contínua simétrica associada ao polinômio $P$. Então $T_{P}$ é linear e continua.

Demonstração: Facilmente vemos que a aplicação $T_{P}$ é linear, pois segue diretamente da multilinearidade da aplicação $A$.

Seja $x \in X$, então:

$$
\left\|T_{P}(x)\right\|=\sup _{\left\|x_{i}\right\| \leq 1 ; 2 \leq i \leq m}\left\|T_{P}(x)\left(x_{2}, \ldots, x_{m}\right)\right\|=\sup _{\left\|x_{i}\right\| \leq 1 ; 2 \leq i \leq m}\left\|A\left(x, x_{2}, \ldots, x_{m}\right)\right\| .
$$


Mas $\left\|A\left(x, x_{2}, \ldots, x_{m}\right)\right\| \leq\|A\|\|x\|\left\|x_{2}\right\| \ldots\left\|x_{m}\right\|$. Então $\left\|T_{P}(x)\right\| \leq\|A\|\|x\|$. Portanto $T_{P}$ é contínua.

Observação: A aplicação linear $T_{P}$ definida acima é denominada de aplicação linear associada ao polinômio $P$. Como $A$ é única então $T_{P}$ também é única.

O próximo teorema foi demonstrado por Aron, Hervés \& Valdivia em [4]. Ele nos dá uma relação entre os polinômios fracamente contínuos com a sua aplicação linear associada.

Teorema 2.5.8. Sejam $X, Y$ espaços de Banach e $m \in \mathbb{N}$. Seja $P \in \mathcal{P}\left({ }^{m} X ; Y\right)$ e seja $T_{P}: X \longrightarrow \mathcal{L}_{s}\left({ }^{m-1} X ; Y\right)$ a aplicação linear associada ao polinômio $P$. Então $P \in \mathcal{P}_{w}\left({ }^{m} X ; Y\right)$ se e somente se $T_{P}$ é compacto.

Demonstração: $(\Rightarrow)$ Seja $P \in \mathcal{P}_{w}\left({ }^{m} X ; Y\right)$. Pela proposição 1.2 .31 basta mostrar que $T_{P}$ é fracamente contínua sobre os limitados de $X$.

Sejam $W \subset X$ limitado, $x \in W$ e $\epsilon>0$. Como $P \in \mathcal{P}_{w}\left({ }^{m} X ; Y\right)$ existem $\phi_{1}, \ldots, \phi_{n} \in X^{*}$ e $\delta>0$ tal que $\|P(x)-P(y)\|<\frac{m !}{m^{m}} \epsilon$ sempre que $\left|\phi_{i}(x-y)\right|<\delta$ com $y \in W$ e $i=1, \ldots, n$. Sejam $A$ a aplicação m-linear simétrica associada ao polinômio $P$ e $z_{2}, \ldots, z_{m} \in W$, pela fórmula da Polarização temos

$$
\begin{gathered}
{\left[T_{P}(x)-T_{P}(y)\right]\left(z_{2}, \ldots, z_{m}\right)=A\left(x, z_{2}, \ldots, z_{m}\right)-A\left(y, z_{2}, \ldots, z_{m}\right)} \\
=\frac{m^{m}}{m ! 2^{m}} \sum_{\epsilon_{i}= \pm 1 ; 1 \leq i \leq m} \epsilon_{1} \ldots \cdot \epsilon_{m}\left[P\left(\frac{\epsilon_{1} x+\epsilon_{2} z_{2}+\ldots+\epsilon_{m} z_{m}}{m}\right)-P\left(\frac{\epsilon_{1} y+\epsilon_{2} z_{2}+\ldots+\epsilon_{m} z_{m}}{m}\right)\right] .
\end{gathered}
$$

Como, para cada $i=1, \ldots, n$,

$$
\phi_{i}\left(\frac{\epsilon_{1} x+\epsilon_{2} z_{2}+\ldots+\epsilon_{m} z_{m}}{m}-\frac{\epsilon_{1} y+\epsilon_{2} z_{2}+\ldots+\epsilon_{m} z_{m}}{m}\right)=\phi_{i}\left(\frac{\epsilon_{1}}{m}(x-y)\right)
$$

então

$$
\left|\phi_{i}\left(\frac{\epsilon_{1} x+\epsilon_{2} z_{2}+\ldots+\epsilon_{m} z_{m}}{m}-\frac{\epsilon_{1} y+\epsilon_{2} z_{2}+\ldots+\epsilon_{m} z_{m}}{m}\right)\right|=\frac{1}{m}\left|\phi_{i}(x-y)\right| .
$$

Logo, tomando $\delta^{*}=m \delta$ se $y \in W$ é tal que $\left|\phi_{i}(x-y)\right|<\delta^{*}$ para cada $i=1, \ldots, n$, temos que

$$
\left|\phi_{i}\left(\frac{\epsilon_{1} x+\epsilon_{2} z_{2}+\ldots+\epsilon_{m} z_{m}}{m}-\frac{\epsilon_{1} y+\epsilon_{2} z_{2}+\ldots+\epsilon_{m} z_{m}}{m}\right)\right|<\delta,
$$

para cada $i=1, \ldots, n, \operatorname{logo}$

$$
\begin{gathered}
\| m^{m} \\
m ! 2^{m} \\
\sum_{\epsilon_{i}= \pm 1 ; 1 \leq i \leq m}(x)-T_{P}(y) \| \leq \\
m P\left(\frac{\epsilon_{1} x+\epsilon_{2} z_{2}+\ldots+\epsilon_{m} z_{m}}{m}\right)-P\left(\frac{\epsilon_{1} y+\epsilon_{2} z_{2}+\ldots+\epsilon_{m} z_{m}}{m}\right) \|
\end{gathered}
$$




$$
<\frac{m^{m}}{m ! 2^{m}} 2^{m} \frac{1}{m^{m}} m ! \epsilon=\epsilon .
$$

Assim, $\left\|T_{P}(x)-T_{P}(y)\right\|<\epsilon$.

Desta maneira, $T_{P}$ é fracamente contínua. Portanto $T_{P}$ é compacta.

$(\Leftarrow)$ Sejam $P \in \mathcal{P}\left({ }^{m} X ; Y\right)$ e $A$ a aplicação m-linear simétrica contínua associada ao polinômio $P$. Vamos supor que $T_{P}$ é compacto e provemos que $P$ é fracamente contínuo sobre os limitados. Pela proposição 2.5.5, basta mostrar que $P$ é fracamente contínua em $B_{X}$. Sejam $x \in B_{X}$ e $\epsilon>0$. Como $T_{P}$ é compacto, então pelo teorema 1.2.31, $T_{P}$ é fracamente contínuo sobre $B_{X}$, logo existem $\delta>0$ e $\phi_{1}, \ldots, \phi_{n} \in X^{*}$ tal que $\left\|T_{P}(x)-T_{P}(y)\right\|<\frac{\epsilon}{m}$ sempre que $\left|\phi_{i}(x-y)\right|<\delta \operatorname{com} y \in B_{X}$. Mas

$$
\begin{aligned}
\|P(x)-P(y)\|= & \|A(x, \stackrel{(m)}{*}, x)-A(y, \stackrel{(m)}{.}, y)\| \\
\leq & \|A(x-y, x, \stackrel{(m-1)}{\cdots}, x)\|+\left\|A\left(x, x-y, x,{ }^{(m-2)}, x\right)\right\| \\
& +\ldots+\|A(y, \stackrel{(m-1)}{\cdots}, y, x-y)\| .
\end{aligned}
$$

Como $A$ é simétrica, então

$$
\begin{gathered}
A(x, \ldots, x-y, x, \ldots, x)=T_{P}(x-y)(x, \ldots, x, y, \ldots, y) \\
=\left(T_{P}(x)-T_{P}(y)\right)(x, \ldots, x, y, \ldots, y) .
\end{gathered}
$$

Assim, se $\left|\phi_{i}(x-y)\right|<\delta$ com $y \in B_{X}$ para todo $i=1, \ldots, n$, temos que

$$
\|P(x)-P(y)\|<\frac{m \epsilon}{m}=\epsilon .
$$

Logo $P$ é fracamente contínua sobre $B_{X}$. Portanto $P \in \mathcal{P}_{w}\left({ }^{m} X ; Y\right)$.

Corolário 2.5.9. Sejam $X, Y$ espaços de Banach e $m \in \mathbb{N}$. Então

$$
\mathcal{P}_{w u}\left({ }^{m} X ; Y\right)=\mathcal{P}_{w}\left({ }^{m} X ; Y\right) .
$$

Demonstração: A inclusão $\mathcal{P}_{w u}\left({ }^{m} X ; Y\right) \subset \mathcal{P}_{w}\left({ }^{m} X ; Y\right)$ é imediata.

Sejam $P \in \mathcal{P}_{w}\left({ }^{m} X ; Y\right)$ e $T_{P}$ a aplicação linear associada ao polinômio $P$. Pelo teorema 2.5.8 temos que $T_{P}$ é compacta, logo pelo teorema 1.2.31 temos que $T_{P}$ é fracamente uniformemente contínua sobre os limitados. Assim dado $\epsilon>0$ existem $\phi_{1}, \ldots, \phi_{n} \in X^{*}$ e $\delta>0$ tal que $\left\|T_{P}(x)-T_{P}(y)\right\|<\frac{\epsilon}{m}$ sempre que $\left|\phi_{i}(x-y)\right|<\delta \operatorname{com} x, y \in B_{X}$ e $i=1, \ldots, n$. Seja $A$ a aplicação m-linear simétrica contínua associada ao polinômio $P$, então:

$$
\|P(x)-P(y)\| \leq\|A(x, x-y, x, \stackrel{(m-2)}{\cdots}, x)\|+\ldots+\|A(y, \stackrel{(m-1)}{\cdots}, y, x-y)\| .
$$


Como $A$ é simétrica, então:

$$
\begin{aligned}
A(x, \ldots, x-y, x, \ldots, x) & =T_{P}(x-y)(x, \ldots, x, y, \ldots, y) \\
& =\left(T_{P}(x)-T_{P}(y)\right)(x, \ldots, x, y, \ldots, y) .
\end{aligned}
$$

Assim, se $\left|\phi_{i}(x-y)\right|<\delta$ com $x, y \in B_{X}$ para todo $i=1, \ldots, n$, temos que $\|P(x)-P(y)\|<\frac{m \epsilon}{m}=\epsilon$. Logo $P$ é fracamente uniformemente contínuo sobre $B_{X}$. Portanto $\mathcal{P}_{w u}\left({ }^{m} X ; Y\right)=\mathcal{P}_{w}\left({ }^{m} X ; Y\right)$.

Proposição 2.5.10. Sejam X, Y espaços de Banach, $m \in \mathbb{N}$. Então

$$
\mathcal{P}_{w u}\left({ }^{m} X ; Y\right) \subset \mathcal{P}_{w s c}\left({ }^{m} X ; Y\right)
$$

Demonstração: Sejam $P \in \mathcal{P}_{w u}\left({ }^{m} X ; Y\right)$ e $\left(x_{n}\right)_{n} \subset X$ uma sequência fracamente convergente para $x$. Assim $W=\left\{x_{n}, x: n \in \mathbb{N}\right\}$ é um conjunto limitado em X e consequentemente $\left.P\right|_{W}$ é fracamente uniformemente contínuo. Logo dado $\epsilon>0$ existem $\phi_{1}, \ldots, \phi_{k} \in X^{*}$ e $\delta>0$ tais que $\|P(y)-P(z)\|<\epsilon$ sempre que $\left|\phi_{i}(y-z)\right|<\delta$ $(i=1, \ldots, k)$ e $y, z \in W$. Como $x_{n} \stackrel{\omega}{\rightarrow} x$, para cada $i=1, \ldots, k$ existe $n_{i} \in \mathbb{N}$ tal que $\left|\phi_{i}\left(x_{n}\right)-\phi_{i}(x)\right|<\delta$ para todo $n \geq n_{i}$. Tomando $n_{0}=\max \left\{n_{1}, \ldots, n_{k}\right\}$ temos que para cada $i=1, \ldots, k,\left|\phi_{i}\left(x_{n}\right)-\phi_{i}(x)\right|<\delta$ para todo $n \geq n_{0}$. Logo, $\left\|P\left(x_{n}\right)-P(x)\right\|<\epsilon$ para todo $n \geq n_{0}$. Portanto $P\left(x_{n}\right) \stackrel{n \rightarrow \infty}{\longrightarrow} P(x)$.

No próximo exemplo, mostramos que nem sempre vale a igualdade.

Exemplo 2.5.11. Seja $P \in \mathcal{P}\left({ }^{2} l_{1}\right)$ ou $P \in \mathcal{P}\left({ }^{2} l_{2}\right)$ dado por:

$$
P(x)=\sum_{n=1}^{\infty} x_{n}^{2} .
$$

Afrmamos que:

1. $P \notin \mathcal{P}_{w s c}\left({ }^{2} l_{2}\right)$.

2. $P \in \mathcal{P}_{w s c}\left({ }^{2} l_{1}\right)$.

3. $P \notin \mathcal{P}_{w u}\left({ }^{2} l_{1}\right)$.

De fato: (1) Seja $\left(e_{j}\right)_{j}$ a base canônica de $l_{2}$, então $e_{j} \stackrel{\omega}{\rightarrow} 0$. Agora, para cada $j \in \mathbb{N}$ temos que $P\left(e_{j}\right)=\sum_{n=1}^{\infty} \delta_{j n}^{2}=1^{2}=1$. Portanto $P \notin \mathcal{P}_{w s c}\left({ }^{2} l_{2}\right)$.

(2) Sejam $x \in X$ e $\left(x_{n}\right)_{n} \subset l_{1}$ uma sequência tal que $x_{n} \stackrel{\omega}{\rightarrow} x$. Como $l_{1}$ é Schur então 
$x_{n} \stackrel{n \rightarrow \infty}{\longrightarrow} x$ e consequentemente $P\left(x_{n}\right) \stackrel{n \rightarrow \infty}{\longrightarrow} P(x)$. Portanto $P \in \mathcal{P}_{w s c}\left({ }^{2} l_{1}\right)$.

(3) Seja $\left(e_{j}\right)_{j}$ a base canônica de $l_{1}$ e considere a sequência $\left(\frac{1}{2} e_{j}\right)_{j}$. Então $e_{j}-\frac{1}{2} e_{j}=\frac{1}{2} e_{j} \stackrel{\omega}{\rightarrow} 0$. Para cada $\delta>0$ e $\left\{\phi_{1}, \ldots, \phi_{n}\right\} \subset l_{1}^{*}$ finita, existe $n_{0} \in \mathbb{N}$ tal que para cada $k=1, \ldots, n,\left|\phi_{k}\left(e_{j}\right)-\phi_{k}\left(\frac{1}{2} e_{j}\right)\right|<\delta$ para todo $j \geq n_{0}$. Agora, para a bola unitária fechada $B_{l_{1}}$, tomando $\epsilon=\frac{1}{4}$ temos que para cada $j \in \mathbb{N}$, $\left|P\left(e_{j}\right)-P\left(\frac{1}{2} e_{j}\right)\right|=\left|1-\left(\frac{1}{2}\right)^{2}\right|=\frac{3}{4} \geq \epsilon$, ou seja, $P$ não uniformemente contínuo sobre $B_{l_{1}}$. Portanto $P \notin \mathcal{P}_{w u}\left({ }^{2} l_{1}\right)$.

Definição 2.5.12. Sejam $X, Y$ espaços de Banach, $m \in \mathbb{N}$ e $P \in \mathcal{P}\left({ }^{m} X ; Y\right)$. Dizemos que $P$ é um polinômio de tipo finito se $P$ é uma combinação linear finita da coleção de funções

$$
\left\{\phi^{m} \otimes y: \phi \in X^{*}, y \in Y\right\}
$$

onde para cada $x \in X,\left(\phi^{m} \otimes y\right)(x)=\phi^{m}(x) \cdot y$.

Vamos denotar por $\mathcal{P}_{f}\left({ }^{m} X ; Y\right)$ o subespaço vetorial de $\mathcal{P}\left({ }^{m} X ; Y\right)$ formado pelos polinômios $\mathrm{m}$-homogêneos de tipo finito. $\mathrm{E} \mathcal{P}_{f}(X ; Y)$ o espaço vetorial de todos os polinômios contínuos de tipo finito, ou seja,

$$
\mathcal{P}_{f}(X ; Y)=\sum_{m=0}^{\infty} \mathcal{P}_{f}\left({ }^{m} X ; Y\right)
$$

Exemplos 2.5.13. Para cada $m \in \mathbb{N}$, sejam $P: l_{2} \longrightarrow \mathbb{K}$ e $Q: l_{2} \longrightarrow \mathbb{K}$ dados por:

$$
P(x)=\sum_{j=1}^{m} x_{j}^{2} \quad e \quad Q(x)=\sum_{j=1}^{\infty} x_{j}^{2}
$$

para cada $x=\left(x_{j}\right)_{j} \in l_{2}$. Afirmamos que $P$ é um polinômio de tipo finito e $Q$ não é de tipo finito.

De fato: Para cada $j=1, \ldots, m$ seja $f_{j} \in l_{2}^{*}$ dado por $f_{j}(x)=f_{j}\left(\left(x_{i}\right)_{i}\right)=x_{j}$ para cada $x=\left(x_{i}\right)_{i} \in l_{2}$. Assim,

$$
P(x)=\sum_{j=1}^{m} x_{j}^{2}=\sum_{j=1}^{m} f_{j}^{2}(x)
$$


Portanto $P$ é um polinômio de tipo finito.

De maneira análoga ao item (3) do exemplo 2.5.11 temos que $Q$ não é fracamente uniformemente contínuo em $B_{l_{2}}$, ou seja, $Q \notin \mathcal{P}_{w u}\left({ }^{2} l_{2}\right)$. Como $l_{2}^{*}$ tem a propriedade da aproximação segue de [[4], corolário 2.11] que $Q \notin \overline{\mathcal{P}_{f}\left({ }^{2} l_{2}\right)}$. Portanto $Q$ não é um polinômio de tipo finito.

Um dos primeiros resultados relacionando polinômios (ou aplicações multilineares) com as propriedades de convergência fraca de sequências foi feito por Littlewood em [26], onde ele mostrou que toda aplicação bilinear limitada $A: c_{0} \longrightarrow \mathbb{K}$ é aproximável por aplicações de tipo finito. Estes resultados foram extendidos por Bogdanowzki e Pelczynski para aplicações $m$-lineares, para cada $m \geq 1$. Assim, temos o seguinte teorema:

Teorema 2.5.14. Seja $m \in \mathbb{N}$ então o subespaço $\mathcal{P}_{f}\left({ }^{m} c_{0}\right)$ é denso no espaço $\mathcal{P}\left({ }^{m} c_{0}\right)$.

É conhecido como teorema de Littewood-Bogdanowzki-Pelczynski. A demonstração do teorema pode ser encontrada, por exemplo, em [17]. 


\section{Capítulo 3}

\section{Propriedade $\mathbf{P}$}

\subsection{Introdução}

Neste capítulo temos por objetivo estudar uma propriedade que foi introduzida por Aron, Choi \& Llavona em [2] denominada de propriedade P. No desenvolvimento do trabalho, vamos estudar as relações da propriedade $\mathbf{P}$ com outras propriedades de espaços de Banach, tais como: propriedade de Schur, Dunford-Pettis e polinomialmente Schur. Também apresentamos exemplos de espaços de Banach que possuam a propriedade $\mathbf{P}$ e um exemplo de um espaço de Banach que não possui tal propriedade.

As propriedades Dunford-Pettis e polinomialmente Schur foram estudadas por Diestel [14] e Farmer \& Johnson [16], Ryan [29] respectivamente. Estas propriedades também foram tema de dissertação de mestrado de Adriana J. Hissadomi [21], e este é um texto que estudamos e vamos utilizá-lo como referência para as demonstrações dos resultados citados.

A seguir apresentamos as definições de tais resultados e principais teoremas que vamos utilizar neste nosso trabalho.

Definição 3.1.1. Seja $X$ um espaço de Banach. Dizemos que $X$ é um espaço polinomialmente Schur se para cada sequência $\left(x_{n}\right)_{n}$ em $X$ com $P\left(x_{n}\right) \stackrel{n \rightarrow \infty}{\longrightarrow} 0$ para cada $P \in \mathcal{P}(X)$ temos que $\left\|x_{n}\right\| \rightarrow 0$.

A seguir vamos dar alguns exemplos de espaços que são polinomialmente Schur e espaços que não são. 
Exemplos 3.1.2. $\quad$ 1. Os espaços $c_{0}, l_{\infty}, L_{1}[0,1]$ e $L_{\infty}[0,1]$ não são polinomialmente Schur.

2. Para $p, 1 \leq p<\infty, l_{p}$ é polinomialmente Schur.

3. Para $q, 2 \leq q<\infty, L_{q}[0,1]$ é polinomialmente Schur.

4. Seja X um espaço de Banach. Se X é de tipo não-trivial então X é polinomialmente Schur.

As demonstrações de que os espaços descritos nos items 1, 2 e 3 são (ou não são) polinomialmente Schur se encontram em Hissadomi, [21] pgs 103 e 120, 110 e 113 respectivamente. E a afirmação 4 foi demonstrada por Farmer \& Jonshon em [16].

\section{Definição 3.1.3. (Dunford-Pettis)}

Seja $X$ um espaço de Banach. Dizemos que $X$ tem a propriedade Dunford-Pettis se dado qualquer espaço de Banach $Y$ e qualquer aplicação linear fracamente compacta $A: X \longrightarrow Y A$ aplica sequências fracamente Cauchy de $X$ em sequências de Cauchy em $Y$.

Proposição 3.1.4. Seja X um espaço de Banach.

1. Se $X$ é reflexivo então $X$ tem a propriedade Dunford-Pettis se e somente se $\operatorname{dim} X<\infty$.

2. Se $X$ é Schur então $X$ tem a propriedade Dunford-Pettis.

Demonstração: Ver corolários 3.4 e 3.6, pgs 63 e 64 respect/, de Hissadomi, [21].

Teorema 3.1.5. Seja $X$ um espaço de Banach. Então $X$ é Schur se e somente se $X$ tem a propriedade Dunford-Pettis e é polinomialmente Schur.

Demonstração: Ver teorema 4.19, pg 120, de Hissadomi, [21].

Apresentamos agora alguns exemplos de espaços que têm a propriedade Dunford-Pettis e espaços que não a possuem.

Exemplos 3.1.6. 1. Os espaços $c_{0}, l_{1}, l_{\infty}, L_{\infty}[0,1], L_{1}[0,1], L_{1}[0,2 \pi]$ e $C[0,1]$ têm a propriedade Dunford-Pettis. 
2. Para $1<p<\infty$, os espaços $l_{p}$ e $L_{p}[0,1]$ não têm a propriedade Dunford-Pettis.

As demonstrações de (1) e (2) se encontram em Hissadomi, [21].

Proposição 3.1.7. Seja X um espaço de Banach com a propriedade Dunford-Pettis. Então valem as seguintes afirmações:

1. Todo polinômio contínuo $P: X \longrightarrow \mathbb{K}$ é fracamente sequencialmente contínuo, isto é, $\mathcal{P}(X)=\mathcal{P}_{w s c}(X)$.

2. Para cada $r \geq 1$, se para cada par de sequências $\left(P_{n}\right)_{n} \subset \mathcal{P}\left({ }^{r} X\right)$ e $\left(x_{n}\right)_{n} \subset X$, com $P_{n} \stackrel{\omega}{\rightarrow} 0$ e $P\left(x_{n}\right) \stackrel{n \rightarrow \infty}{\longrightarrow} 0$ para cada $P \in \mathcal{P}(X)$, temos que $P_{n}\left(x_{n}\right) \stackrel{n \rightarrow \infty}{\longrightarrow} 0$.

Demonstração: Para 1) ver Hissadomi, [21] corolário 3.46 e para 2) ver [8].

\subsection{Propriedade $\mathbf{P}$}

A motivação que originou a definição da propriedade $\mathbf{P}$ é a seguinte:

Sejam $X, Y$ espaços de Banach sobre o corpo $\mathbb{K}$ onde $B_{X}, B_{Y}$ são as bolas unitárias fechadas de $X$ e $Y$ respectivamente, e $g: B_{X} \longrightarrow B_{Y}$ uma função que satisfaz a seguinte propriedade $(\star)$ :

(*) Para qualquer polinômio contínuo $P: Y \longrightarrow \mathbb{K}, P \circ g: B_{X} \longrightarrow \mathbb{K}$ é uniformemente continua.

Seria g necessariamente uniformemente contínua?

Se $g$ satisfaz $(\star)$, Aron, Choi \& Llavona, no artigo [2], observaram que o problema tem resposta positiva se a seguinte condição é satisfeita:

(**): Dados duas sequências limitadas $\left(u_{n}\right)_{n},\left(v_{n}\right)_{n}$ em $Y$, se $\left|P\left(u_{n}\right)-P\left(v_{n}\right)\right| \stackrel{n \rightarrow \infty}{\longrightarrow} 0$ para todo $P \in \mathcal{P}(Y)$ segue que $\left\|u_{n}-v_{n}\right\| \stackrel{n \rightarrow \infty}{\longrightarrow} 0$.

Mostramos tal observação na seguinte proposição: 
Proposição 3.2.1. Sejam $X, Y$ espaços de Banach sobre o corpo $\mathbb{K}$ onde $B_{X}, B_{Y}$ são as bolas unitárias fechadas de $X$ e $Y$ respectivamente, e $g: B_{X} \longrightarrow B_{Y}$ uma função tal que satisfaz a propriedade ( $\star$ ). Se $Y$ satisfaz a propriedade ( $\star \star)$ então g é uniformemente contínua.

Demonstração: Sejam $\left(x_{n}\right)_{n},\left(y_{n}\right)_{n}$ sequências em $B_{X}$ tais que $\left\|x_{n}-y_{n}\right\| \stackrel{n \rightarrow \infty}{\longrightarrow} 0$. Como $g$ satisfaz a propriedade $(\star)$ então do teorema 1.2 .29 segue que para cada $P \in \mathcal{P}(Y)$, $\left|P\left(g\left(x_{n}\right)\right)-P\left(g\left(y_{n}\right)\right)\right| \stackrel{n \rightarrow \infty}{\longrightarrow} 0$. Por hipótese, $Y$ satisfaz $(\star \star)$, então $\left\|g\left(x_{n}\right)-g\left(y_{n}\right)\right\| \stackrel{n \rightarrow \infty}{\longrightarrow} 0$ e consequentemente, pelo teorema 1.2 .29 , temos que $g$ é uniformemente contínua.

No que segue vamos apresentar uma proposição que nos fornece um exemplo de uma função $g$ que não é uniformemente contínua, mas que $P \circ g$ é uniformemente contínua para todo polinômio. Na demonstração da proposição vamos usar o fato de que o espaço $\mathcal{P}_{f}\left({ }^{m} c_{0}\right)$ é denso no espaço $\mathcal{P}\left({ }^{m} c_{0}\right), m \geq 1$ (ver teorema 2.5.14).

Proposição 3.2.2. Seja $g: B_{c_{0}} \longrightarrow B_{c_{0}}$ a função dada por

$$
g(x)=g\left(\left(x_{n}\right)\right)=\left(x_{n}^{n}\right)_{n} \quad \text { para cada } \quad x=\left(x_{n}\right) \in c_{0} .
$$

Então valem as seguintes afirmações:

1. A função g não é uniformemente contínua.

2. Para quaisquer duas sequências $\left(u_{j}\right)_{j},\left(v_{j}\right)_{j} \in B_{c_{0}}$ com $\left\|u_{j}-v_{j}\right\| \stackrel{j \rightarrow \infty}{\longrightarrow 0}$ temos que $g\left(u_{j}\right)-g\left(v_{j}\right) \stackrel{\omega}{\longrightarrow} 0$. Além disso, $f^{p} \circ g\left(u_{j}\right)-f^{p} \circ g\left(v_{j}\right) \stackrel{j \rightarrow \infty}{\longrightarrow} 0$ para cada $f \in c_{0}^{*}$ e cada $p \geq 2$.

3. A função g satisfaz a condição (*).

Demonstração: (1) Para cada $n \in \mathbb{N}$, seja $e_{n}$ o n-ésimo elemento da base canônica de $c_{0}$, então temos que:

As sequências $\left(e_{n}\right)_{n},\left(\left(1+\frac{1}{n}\right) e_{n}\right)_{n}$ são limitadas e

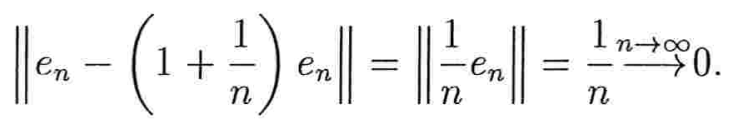

Mas, para cada $n \in \mathbb{N}$, temos:

$$
\left\|g\left(e_{n}\right)-g\left(\left(1+\frac{1}{n}\right)\left(e_{n}\right)\right)\right\|=\sup _{n}\left\|\left(\delta_{i n}^{i}\right)_{i}-\left(\left(\left(1+\frac{1}{n}\right) \delta_{i n}\right)^{i}\right)_{i}\right\| .
$$


Assim,

$$
\left\|g\left(e_{n}\right)-g\left(\left(1+\frac{1}{n}\right)\left(e_{n}\right)\right)\right\|=\left|1-\left(1+\frac{1}{n}\right)^{n}\right| .
$$

Como $\left(1+\frac{1}{n}\right)^{n} \stackrel{n \rightarrow \infty}{\longrightarrow} e$, então

$$
\left\|g\left(e_{n}\right)-g\left(\left(1+\frac{1}{n}\right)\left(e_{n}\right)\right)\right\| \stackrel{n \rightarrow \infty}{\longrightarrow}|1-e|>0 .
$$

Portanto $g$ não é uniformemente contínua.

(2) Sejam $f \in c_{0}^{*}$ e $\left(u_{j}\right)_{j},\left(v_{j}\right)_{j} \in B_{c_{0}}$ tais que $\left\|u_{j}-v_{j}\right\| \stackrel{j \rightarrow \infty}{\longrightarrow 0}$. Como $c_{0}^{*} \cong l_{1}$, $f=\left(f_{1}, f_{2}, \ldots\right) \in l_{1}, f\left(\left(x_{l}\right)_{l}\right)=\sum_{l=1}^{\infty} x_{l} f_{l}$ para cada $\left(x_{l}\right)_{l} \in c_{0}$ e $\sum_{l=1}^{\infty}\left|f_{l}\right|<\infty$, isto é, existe $M>0$ tal que $\left|f_{l}\right|<M$ para cada $l \in \mathbb{N}$ e para $\epsilon>0$ existe $n_{f} \in \mathbb{N}$ tal que $\sum_{k=n_{f}+1}^{\infty}\left|f_{k}\right|<\frac{\epsilon}{4}$. Considere $\epsilon^{*}=\frac{\epsilon}{M n_{f}\left(n_{f}+1\right)}$, como $\left\|u_{j}-v_{j}\right\| \stackrel{j \rightarrow \infty}{\longrightarrow} 0$ então existe $j_{0} \in \mathbb{N}$ tal que $\left\|u_{j}-v_{j}\right\|<\epsilon^{*}$ para cada $j \geq j_{0}$. Se denotarmos $u_{j}=\left(u_{j k}\right)_{k}, v_{j}=\left(v_{j k}\right)_{k}$, temos que $\left|u_{j k}-v_{j k}\right|<\epsilon^{*}$ para cada $k \in \mathbb{N}$ e cada $j \geq j_{0}$.

Para cada $j \in \mathbb{N}$, valem as seguintes desigualdades:

$$
\begin{gathered}
\left|f\left(g\left(u_{j}\right)\right)-f\left(g\left(v_{j}\right)\right)\right| \leq \sum_{k=1}^{n_{f}}\left|u_{j k}^{k}-v_{j k}^{k}\right| \cdot\left|f_{k}\right|+\sum_{k=n_{f}+1}^{\infty}\left|u_{j k}^{k}-v_{j k}^{k}\right| \cdot\left|f_{k}\right| \\
\text { e } \quad \sum_{k=1}^{n_{f}}\left|u_{j k}^{k}-v_{j k}^{k}\right| \cdot\left|f_{k}\right| \leq M \sum_{k=1}^{n_{f}}\left|u_{j k}^{k}-v_{j k}^{k}\right| .
\end{gathered}
$$

Para cada $j \in \mathbb{N}$ e cada $k \in \mathbb{N}$ :

$$
\left|u_{j k}^{k}-v_{j k}^{k}\right|=\left|u_{j k}-v_{j k}\right| \cdot\left|u_{j k}^{k-1}+u_{j k}^{k-2} v_{j k}+\ldots+v_{j k}^{k-1}\right| .
$$

Por hipótese, $u_{j} \in B_{c_{0}}$ e $v_{j} \in B_{c_{0}}$, logo para cada $j \geq j_{0}$,

$$
\begin{gathered}
M \sum_{k=1}^{n_{f}}\left|u_{j k}^{k}-v_{j k}^{k}\right| \leq M \sum_{k=1}^{n_{f}} k\left|u_{j k}-v_{j k}\right|<M \frac{n_{f}\left(n_{f}+1\right)}{2} \cdot \epsilon^{*}=\frac{\epsilon}{2} \\
\mathrm{e} \quad \sum_{k=n_{f}+1}^{\infty}\left|u_{j k}^{k}-v_{j k}^{k}\right| \cdot\left|f_{k}\right| \leq \sup _{n_{f}+1 \leq k \leq \infty}\left|u_{j k}^{k}-v_{j k}^{k}\right| \sum_{k=n_{f}+1}^{\infty}\left|f_{k}\right|<2 \frac{\epsilon}{4}=\frac{\epsilon}{2} .
\end{gathered}
$$

Assim, para cada $j \geq j_{0}$,

$$
\left|f\left(g\left(u_{j}\right)\right)-f\left(g\left(v_{j}\right)\right)\right| \leq \frac{\epsilon}{2}+\frac{\epsilon}{2}=\epsilon .
$$


Portanto $g\left(u_{j}\right)-g\left(v_{j}\right) \stackrel{\omega}{\rightarrow} 0$.

Agora como para cada $f \in c_{0}^{*}$, cada $p \geq 2$ e cada $j \in \mathbb{N}$ temos que:

$$
\begin{aligned}
& f^{p} \circ g\left(u_{j}\right)-f^{p} \circ g\left(v_{j}\right)= \\
& {\left[f\left(g\left(u_{j}\right)\right)-f\left(g\left(v_{j}\right)\right)\right] \cdot\left[f^{p-1}\left(g\left(u_{j}\right)\right)+f^{p-2}\left(g\left(u_{j}\right)\right) f\left(g\left(v_{j}\right)\right)+\ldots+f^{p-1}\left(g\left(v_{j}\right)\right)\right],}
\end{aligned}
$$

e $g\left(u_{j}\right), g\left(v_{j}\right) \in B_{c_{0}}$, segue que

$$
\left|f^{p} \circ g\left(u_{j}\right)-f^{p} \circ g\left(v_{j}\right)\right| \leq p \cdot\|f\|^{p-1}\left|f\left(g\left(u_{j}\right)\right)-f\left(g\left(v_{j}\right)\right)\right| .
$$

Consequentemente $f^{p} \circ g\left(u_{j}\right)-f^{p} \circ g\left(v_{j}\right) \stackrel{j \rightarrow \infty}{\longrightarrow} 0$ para todo $f \in c_{0}^{*}$, uma vez que $g\left(u_{j}\right)-g\left(v_{j}\right) \stackrel{\omega}{\rightarrow} 0$.

(3) Seja $P: c_{0} \longrightarrow \mathbb{K}$ um polinômio contínuo. Vamos mostrar que $P \circ g$ é uniformemente contínua, isto é, se $\left(u_{j}\right)_{j}$ e $\left(v_{j}\right)_{j}$ são sequências em $B_{c_{0}}$ tais que $\left\|u_{j}-v_{j}\right\| \stackrel{j \rightarrow \infty}{\longrightarrow} 0$, vamos provar que $\left|P \circ g\left(u_{j}\right)-P \circ g\left(v_{j}\right)\right| \stackrel{j \rightarrow \infty}{\longrightarrow} 0$. Por definição de polinômio, existem $m \in \mathbb{N}$ e $P_{r} \in \mathcal{P}\left({ }^{r} c_{0}\right), r=0,1, \ldots, m$, tais que

$$
P=P_{0}+P_{1}+\ldots+P_{m}
$$

Para cada $P_{r} \in \mathcal{P}\left({ }^{r} c_{0}\right)$ existe uma sequência $\left(P_{i}\right)_{i} \subset \mathcal{P}_{f}\left({ }^{r} c_{0}\right)$ tal que $P_{i} \stackrel{i \rightarrow \infty}{\longrightarrow} P_{r}$, uma vez que o espaço $\mathcal{P}_{f}\left({ }^{r} c_{0}\right)$ é denso no espaço $\mathcal{P}\left({ }^{r} c_{0}\right)$ (ver teorema 2.5.14). Assim, para $\epsilon>0$ existe $n_{0} \in \mathbb{N}$ tal que $\left\|P_{n_{0}}-P_{r}\right\| \leq \frac{\epsilon}{3}$. Como $P_{n_{0}} \in \mathcal{P}_{f}\left({ }^{r} c_{0}\right)$, segue que $P_{n_{0}}(x)=\sum_{s=1}^{t_{n_{0}}} \lambda_{s} f_{s}^{r}(x)$, $\operatorname{com} \lambda_{s} \in \mathbb{K}, f_{s} \in c_{0}^{*}$ para cada $s=1, \ldots, t_{n_{0}}$ e para cada $x \in c_{0}$. Agora por (2) segue que $f_{s}^{r} \circ g\left(u_{j}\right)-f_{s}^{r} \circ g\left(v_{j}\right) \stackrel{j \rightarrow \infty}{\longrightarrow 0}$ para cada $f_{s} \in c_{0}^{*}$ e $s=1, \ldots, t_{n_{0}}$. Consequentemente,

$$
P_{n_{0}} \circ g\left(u_{j}\right)-P_{n_{0}} \circ g\left(v_{j}\right)=\sum_{s=1}^{t_{n_{0}}} \lambda_{s}\left[\left(f_{s}^{r} \circ g\right)\left(u_{j}\right)-\left(f_{s}^{r} \circ g\right)\left(v_{j}\right)\right] \stackrel{j \rightarrow \infty}{\longrightarrow} 0,
$$

isto é, existe $j_{n_{0}} \in \mathbb{N}$ tal que $\left|P_{n_{0}}\left(g\left(u_{j}\right)\right)-P_{n_{0}}\left(g\left(v_{j}\right)\right)\right|<\frac{\epsilon}{3}$ para todo $j \geq j_{n_{0}}$. Como

$$
\begin{gathered}
\left|P_{r}\left(g\left(u_{j}\right)\right)-P_{r}\left(g\left(v_{j}\right)\right)\right|= \\
\left|P_{r}\left(g\left(u_{j}\right)\right)-P_{n_{0}}\left(g\left(u_{j}\right)\right)+P_{n_{0}}\left(g\left(u_{j}\right)\right)-P_{r}\left(g\left(v_{j}\right)\right)-P_{n_{0}}\left(g\left(v_{j}\right)\right)+P_{n_{0}}\left(g\left(v_{j}\right)\right)\right| \\
\leq\left|P_{n_{0}}\left(g\left(u_{j}\right)\right)-P_{r}\left(g\left(u_{j}\right)\right)\right|+\left|P_{n_{0}}\left(g\left(v_{j}\right)\right)-P_{r}\left(g\left(v_{j}\right)\right)\right|+\left|P_{n_{0}}\left(g\left(u_{j}\right)\right)-P_{n_{0}}\left(g\left(v_{j}\right)\right)\right|
\end{gathered}
$$

temos que $\left|P_{r}\left(g\left(u_{j}\right)\right)-P_{r}\left(g\left(v_{j}\right)\right)\right| \leq \epsilon$ para cada $j \geq j_{n_{0}}$, uma vez que vale $3.2 \mathrm{e}$

$$
\begin{gathered}
\left|P_{n_{0}}\left(g\left(u_{j}\right)\right)-P\left(g\left(u_{j}\right)\right)\right|<\frac{\epsilon}{3} \\
\text { e } \quad\left|P_{n_{0}}\left(g\left(v_{j}\right)\right)-P\left(g\left(v_{j}\right)\right)\right|<\frac{\epsilon}{3},
\end{gathered}
$$


pelo fato de $\left\|g\left(u_{j}\right)\right\| \leq 1$ e $\left\|g\left(v_{j}\right)\right\| \leq 1$. Logo,

$$
\left|P_{r}\left(g\left(u_{j}\right)\right)-P_{r}\left(g\left(v_{j}\right)\right)\right| \stackrel{j \rightarrow \infty}{\longrightarrow} 0 .
$$

Seja $\delta>0$. Como a demonstração não dependeu do grau de homogenidade de $P_{r}$, temos que para cada $r \in\{0, \ldots, m\}$, de 3.3 existe $j_{r} \in \mathbb{N}$ tal que $\left|P_{r}\left(g\left(u_{j}\right)\right)-P_{r}\left(g\left(v_{j}\right)\right)\right| \leq \frac{\delta}{m+1}$ para cada $j \geq j_{r}$. Seja $\hat{j}=\max \left\{j_{0}, \ldots, j_{m}\right\}$. Para cada $j \geq \hat{j}$, de 3.1 temos que:

$$
\begin{aligned}
\left|P\left(g\left(u_{j}\right)\right)-P\left(g\left(v_{j}\right)\right)\right| & \\
\mid P_{0}\left(g\left(u_{j}\right)\right)-P_{0}\left(g\left(v_{j}\right)\right)+ & P_{1}\left(g\left(u_{j}\right)\right)-P_{1}\left(g\left(v_{j}\right)\right)+\ldots+P_{m}\left(g\left(u_{j}\right)\right)-P_{m}\left(g\left(v_{j}\right)\right) \mid \\
\leq \mid P_{0}\left(g\left(u_{j}\right)\right)- & P_{0}\left(g\left(v_{j}\right)\right)|+\ldots+| P_{m}\left(g\left(u_{j}\right)\right)-P_{m}\left(g\left(v_{j}\right)\right) \mid \\
& \leq \frac{\delta}{m+1}+\stackrel{(m)}{\cdot}+\frac{\delta}{m+1}=\delta,
\end{aligned}
$$

$\operatorname{logo}\left|P\left(g\left(u_{j}\right)\right)-P\left(g\left(v_{j}\right)\right)\right| \stackrel{j \rightarrow \infty}{\longrightarrow} 0$. Portanto $P \circ g$ é uniformemente contínua.

Para poder determinar sob que condições os espaços de Banach têm a propriedade $(\star \star)$, Aron, Choi \& Llavona em [2] introduziram a seguinte propriedade mais fraca que $(\star \star):$

Definição 3.2.3. Seja $X$ um espaço de Banach. Dizemos que $X$ tem a propriedade $\mathbf{P}$, se para quaisquer duas sequências limitadas $\left(u_{j}\right)_{j},\left(v_{j}\right)_{j}$ em $X$ tais que para cada $n \geq 1$ e cada $P \in \mathcal{P}\left({ }^{n} X\right)\left|P\left(u_{j}\right)-P\left(v_{j}\right)\right| \stackrel{j \rightarrow \infty}{\longrightarrow} 0$ segue que $\left|Q\left(u_{j}-v_{j}\right)\right| \stackrel{j \rightarrow \infty}{\longrightarrow 0}$ para cada $m \geq 1$ e cada $Q \in \mathcal{P}\left({ }^{m} X\right)$.

A seguir vamos apresentar os resultados estudados abordando as referidas propriedades. Tais resultados foram demonstrados por Aron, Choi \& Llavona em [2].

Teorema 3.2.4. Seja X um espaço de Banach. Considere as seguintes afirmações:

(a) X tem a propriedade ( $\star \star)$.

(b) X é polinomialmente Schur.

(c) X tem a propriedade Dunford-Pettis.

(d) X tem a propriedade $\mathbf{P}$.

Então temos as seguintes implicações: (a) $\Rightarrow(\mathrm{b}),(\mathrm{a}) \Rightarrow(\mathrm{d}),(\mathrm{c}) \Rightarrow(\mathrm{d})$ e (b) e (d) $\Rightarrow$ (a). 
Demonstração: $(\mathrm{a}) \Rightarrow(\mathrm{b})$

Seja $\left(x_{j}\right)_{j}$ uma sequência em $X$ tal que para cada $m \geq 1$ e cada $P \in \mathcal{P}\left({ }^{m} X\right)$ temos que $P\left(x_{j}\right) \stackrel{j \rightarrow \infty}{\longrightarrow} 0$. Assim, $x_{j} \stackrel{\omega}{\rightarrow} 0$, e consequentemente $\left(x_{j}\right)_{j}$ é limitada. Agora como para cada $j \in \mathbb{N}:$

$$
\left|P\left(x_{j}\right)\right|=\left|P\left(x_{j}\right)-P(0)\right|,
$$

e $X$ tem a propriedade $(\star \star)$ então $\left\|x_{j}\right\| \stackrel{j \rightarrow \infty}{\longrightarrow 0}$. Portanto $X$ é polinomialmente Schur.

(a) $\Rightarrow(d)$

Sejam $\left(u_{j}\right)_{j},\left(v_{j}\right)_{j}$ sequências limitadas em $X$. Suponhamos que para cada $n \geq 1$ e cada $P \in \mathcal{P}\left({ }^{n} X\right)$ temos que $\left|P\left(u_{j}\right)-P\left(v_{j}\right)\right| \stackrel{j \rightarrow \infty}{\longrightarrow} 0$. Como $X$ tem a propriedade ( $\left.\star \star\right)$ então $\left\|u_{j}-v_{j}\right\| \stackrel{j \rightarrow \infty}{\longrightarrow} 0$. Sejam $m \in \mathbb{N}$ e $Q \in \mathcal{P}\left({ }^{m} X\right)$, como $Q$ é contínua então $\left|Q\left(u_{j}-v_{j}\right)\right| \stackrel{j \rightarrow \infty}{\longrightarrow} 0$. Portanto $X$ tem a propriedade $\mathbf{P}$.

(c) $\Rightarrow(\mathrm{d})$

Sejam $\left(u_{j}\right)_{j},\left(v_{j}\right)_{j}$ sequências limitadas em $X$ tais que para cada $m \in \mathbb{N}$ e cada

$P \in \mathcal{P}\left({ }^{m} X\right),\left|P\left(u_{j}\right)-P\left(v_{j}\right)\right| \stackrel{j \rightarrow \infty}{\longrightarrow 0}$. Em particular para $m=1$ e cada $f \in \mathcal{P}\left({ }^{1} X\right)=X^{*}$, $\left|f\left(u_{j}\right)-f\left(v_{j}\right)\right|=\left|f\left(u_{j}-v_{j}\right)\right| \stackrel{j \rightarrow \infty}{\longrightarrow} 0$, ou seja, $u_{j}-v_{j} \stackrel{\omega}{\rightarrow} 0$ quando $j \rightarrow \infty$.

Sejam $n \in \mathbb{N}$ e $Q \in \mathcal{P}\left({ }^{n} X\right)$. Como $u_{j}-v_{j} \stackrel{\omega}{\rightarrow} 0$ e $X$ tem a propriedade Dunford-Pettis, então pela proposição $3.1 .7(1)$ temos que $\left|Q\left(u_{j}-v_{j}\right)\right| \stackrel{j \rightarrow \infty}{\longrightarrow} 0$. Portanto $X$ tem a propriedade $\mathbf{P}$.

$[(\mathrm{b})$ e $(\mathrm{d})] \Rightarrow(\mathrm{a})$

Sejam $\left(u_{j}\right)_{j},\left(v_{j}\right)_{j}$ sequências limitadas em $X$ tais que para cada $P \in \mathcal{P}(X)$,

$\left|P\left(u_{j}\right)-P\left(v_{j}\right)\right| \stackrel{j \rightarrow \infty}{\longrightarrow} 0$. Como $X$ tem a propriedade $\mathbf{P}$ então para cada $n \in \mathbb{N}$, e cada

$Q \in \mathcal{P}\left({ }^{n} X\right)$ temos que $\left|Q\left(u_{j}-v_{j}\right)\right| \stackrel{j \rightarrow \infty}{\longrightarrow} 0, \log$ o $\left\|u_{j}-v_{j}\right\| \stackrel{j \rightarrow \infty}{\longrightarrow} 0$, pois $X$ é polinomialmente Schur. Portanto $X$ satisfaz a propriedade $(\star \star)$.

Corolário 3.2.5. Sejam $X, Y$ espaços de Banach com $B_{X}, B_{Y}$ as bolas unitárias fechadas de $X$ e $Y$ respectivamente. Seja $g: B_{X} \longrightarrow B_{Y}$ uma função que verifica a propriedade ( ). Se $Y$ é um espaço polinomialmente Schur e tem a propriedade $\mathbf{P}$, então $g$ é uniformemente contínua.

Demonstração: Pelo teorema acima, temos que $Y$ tem a propriedade $(\star \star)$. Logo, pelo teorema 3.2.1, $g$ é uniformemente contínua.

Corolário 3.2.6. Seja X um espaço de Banach. Se X é Schur então X tem a propriedade $(\star \star)$.

Demonstração: Suponhamos que $X$ é Schur, então pelo teorema $3.1 .5 X$ é polinomialmente Schur e tem a propriedade Dunford-Pettis. Logo, pelo teorema 3.2.4 temos que 
$X$ tem a propriedade $\mathbf{P}$, e assim, $X$ tem a propriedade $(\star \star)$.

Exemplo 3.2.7. O espaço $l_{1}$ tem a propriedade ( $\left.\star \star\right)$, pois $l_{1}$ é Schur.

Exemplos 3.2.8. Os espaços $c_{0}, l_{1}, l_{\infty}, L_{\infty}[0,1], L_{1}[0,1], L_{1}[0,2 \pi]$ e $C[0,1]$ têm a propriedade $\mathbf{P}$ uma vez que têm a propriedade Dunford-Pettis. A demostração de que tais espaços têm a propriedade Dunford-Pettis pode ser encontrada em [21].

A seguir apresentamos alguns exemplos de espaços de Banach, os quais nos mostram que as únicas relações entre as afirmações (a), (b), (c) e (d) do teorema 3.2 .4 são as fornecidas pelo mesmo.

Exemplos 3.2.9. Os espaços $L_{1}[0,1], c_{0}, l_{\infty}$ e $L_{\infty}[0,1]$ não satisfazem a propriedade

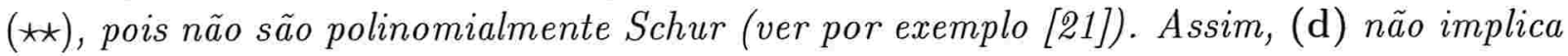
(a) no teorema 3.2.4, pois estes espaços têm a propriedade $\mathbf{P}$.

No teorema 3.2.4 a hipótese (d) não implica (c), pois os espaços $l_{p}$ e $L_{p}[0,1]$, $1<p<\infty$, não são Dunford-Pettis, e a seguir vamos estudar resultados que garantem que estes espaços têm a propriedade $\mathbf{P}$.

Definição 3.2.10. Dizemos que um espaço de Banach $X$ pertence a classe $W_{p}$, $1<p<\infty$, quando cada sequência limitada $\left(x_{n}\right)_{n} \subset X$ admite uma subsequência $\left(x_{n_{k}}\right)_{k}$ fracamente $p$-convergente, isto é, existe $x \in X$ tal que $\sum_{k=1}^{\infty}\left|x^{*}\left(x_{n_{k}}-x\right)\right|^{p}<\infty$ para todo $x^{*} \in X^{*}$.

O próximo teorema foi demonstrado por Aron, Choi \& Llavona, em [2].

Teorema 3.2.11. Seja $X$ um espaço de Banach tal que $X^{*} \in W_{p}$ para algum $p \in(1, \infty)$. Então $X$ tem a propriedade ( $\star \star)$, e consequentemente a propriedade $\mathbf{P}$.

Demonstração: Sejam $\left(x_{n}\right)_{n}$ e $\left(y_{n}\right)_{n}$ sequências limitadas em $X$ tais que para cada $P \in \mathcal{P}(X)$ temos que $\left|P\left(x_{n}\right)-P\left(y_{n}\right)\right| \stackrel{n \rightarrow \infty}{\longrightarrow} 0$. Suponhamos, por absurdo, que $\left\|x_{n}-y_{n}\right\| \nrightarrow \rightarrow$, então existem $\delta>0$ e subsequências de $\left(x_{n}\right)_{n}$ e $\left(y_{n}\right)_{n}$, que vamos denotar, sem perda de generalidade, por $\left(x_{n}\right)_{n}$ e $\left(y_{n}\right)_{n}$ respectivamente, tais que $\left\|x_{n}-y_{n}\right\|>\delta$ para cada $n \in \mathbb{N}$. Por hipótese, a subsequência $\left(x_{n}-y_{n}\right)_{n}$ converge fracamente a zero, assim pelo teorema Bessaga-Pelczynski (1.2.32), admite uma subsequência básica, que vamos 
denotar por $\left(x_{n}-y_{n}\right)_{n}$ (daqui em diante, nesta demonstração, vamos trabalhar com esta sequência). Para cada $n \in \mathbb{N}$ sejam $z_{n}=x_{n}-y_{n}$ e $Z$ o subespaço fechado de $X$ gerado pela sequência $\left(z_{n}\right)_{n}$. Seja $\left(z_{n}^{*}\right)_{n} \subset Z^{*}$ os coeficientes funcionais associados a sequência $\left(z_{n}\right)_{n}$. Assim pelo teorema 1.2 .14 , para cada $n \in \mathbb{N}, z_{n}^{*}$ é um funcional limitado em $Z^{*}$, logo pelo teorema de Hahn-Banach (1.2.6), existe $\hat{z}_{n}^{*} \in X^{*}$ tal que

$$
\left\|z_{n}^{*}\right\|=\left\|\hat{z}_{n}^{*}\right\|
$$

Como $\inf _{n}\left\|z_{n}\right\|>0$ então, pelo teorema 1.2.14, $\left(\hat{z}_{n}^{*}\right)_{n}$ é limitada em $X^{*}$. Agora, por hipótese, $X^{*} \in W_{p}$ então existem $y^{*} \in X^{*}$ e uma subsequência de $\left(\hat{z}_{n}^{*}\right)_{n}$, que vamos denotar por $\left(\hat{z}_{n_{j}}^{*}\right)_{j}$, tal que

$$
\sum_{j=1}^{\infty}\left|x^{* *}\left(\hat{z}_{n_{j}}^{*}-y^{*}\right)\right|^{p}<\infty \quad \text { para cada } x^{* *} \in X^{* *}
$$

Como para cada $x \in X$, a função $J_{x}: X^{*} \longrightarrow \mathbb{K}$ dada por: $J_{x}\left(y^{*}\right)=y^{*}(x)$ para cada $y^{*} \in X^{*}$, pertence a $X^{* *}$, segue que para cada $x \in X, \sum_{j=1}^{\infty}\left|J_{x}\left(\hat{z}_{n_{j}}^{*}-y^{*}\right)\right|^{p}<\infty$. Mas

$$
\begin{gathered}
\sum_{j=1}^{\infty}\left|J_{x}\left(\hat{z}_{n_{j}}^{*}-y^{*}\right)\right|^{p}=\sum_{j=1}^{\infty}\left|\left(\hat{z}_{n_{j}}^{*}-y^{*}\right)(x)\right|^{p} . \text { Logo } \\
\sum_{j=1}^{\infty}\left|\left(\hat{z}_{n_{j}}^{*}-y^{*}\right)(x)\right|^{p}<\infty \quad \text { para cada } x \in X .
\end{gathered}
$$

Assim, para $\epsilon>0$ e cada $x \in X$, temos que existe $j_{x} \in \mathbb{N}$ tal que $\sum_{j=j_{x}}^{\infty}\left|\left(\hat{z}_{n_{j}}^{*}-y^{*}\right)(x)\right|^{p}<\epsilon^{p}$. Logo $\left|\left(\hat{z}_{n_{j}}^{*}-y^{*}\right)(x)\right|^{p}<\epsilon^{p}$ para cada $j \geq j_{x}$, ou seja,

$$
\text { para cada } x \in X, \quad\left(\hat{z}_{n_{j}}^{*}-y^{*}\right)(x) \rightarrow 0
$$

Agora, para cada $z \in Z$, existe uma única sequência de escalares $\left(a_{n}\right)_{n}$ tal que $z=\sum_{n=1}^{\infty} a_{n} z_{n}$, uma vez que $\left(z_{n}\right)_{n}$ é base para $Z$. Tal sequência $\left(a_{n}\right)_{n} \in l_{1}$, isto segue da unicidade de $\left(a_{n}\right)_{n}$ e do teorema de Banach-Mazur (1.2.2) que garante a existência de uma aplicação linear, contínua e sobrejetora $T: l_{1} \longrightarrow Z$ dada por: $T\left(\left(\alpha_{n}\right)_{n}\right)=\sum_{n=1}^{\infty} \alpha_{n} z_{n}$. Como para cada $j \in \mathbb{N}$

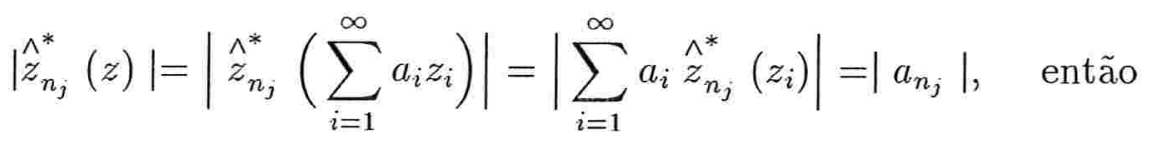




$$
\stackrel{\wedge}{z}_{n_{j}}^{*}(z) \rightarrow 0 \quad \text { para cada } z \in Z
$$

Assim, por 3.7 e 3.8 temos que $y^{*}(z)=0$ para cada $z \in Z$.

Considere $m \in \mathbb{N}$ fixo, para cada $j \in \mathbb{N}$, temos:

$$
\left(\hat{z}_{n_{j}}^{*}-y^{*}\right)\left(x_{m}\right)-\left(\hat{z}_{n_{j}}^{*}-y^{*}\right)\left(y_{m}\right)=\hat{z}_{n_{j}}^{*}\left(z_{m}\right)-y^{*}\left(z_{m}\right)=\delta_{n_{j} m}=\left\{\begin{array}{lll}
1 & \text { se } & n_{j}=m \\
0 & \text { se } & n_{j} \neq m
\end{array} .\right.
$$

A sequência $\left(\left(\hat{z}_{n_{j}}^{*}-y^{*}\right)\left(x_{n_{j}}\right)\right)_{j}$ é limitada em $\mathbb{K}$, onde os $n_{j}$ s são os índices correspondentes aos índices da sequência $\left(\hat{z}_{n_{j}}^{*}\right)_{j}$, logo admite uma subsequência convergente, que vamos denotar por $\left(\left(\hat{z}_{n_{j_{l}}}^{*}-y^{*}\right)\left(x_{n_{j_{l}}}\right)\right)_{l}$, e digamos que $\left(\hat{z}_{n_{j_{l}}}^{*}-y^{*}\right)\left(x_{n_{j_{l}}}\right) \rightarrow$ a para algum $a \in \mathbb{K}$. A sequência $\left(\left(\hat{z}_{n_{j_{l}}}^{\wedge^{*}}-y^{*}\right)\left(y_{n_{j_{l}}}\right)\right)_{l}$ é limitada em $\mathbb{K}$, onde os $n_{j_{l}}$ 's são os índices correspondentes aos índices da sequência $\left(\left(\hat{z}_{n_{j_{l}}}^{*}-y^{*}\right)\left(x_{n_{j_{l}}}\right)\right)_{l}$, logo admite uma subsequência

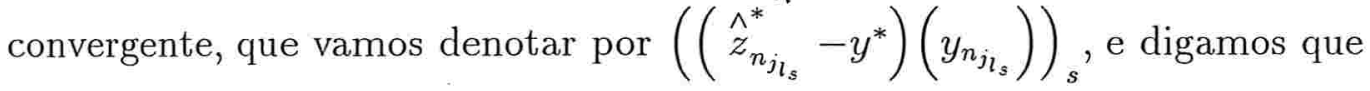

$$
\left(\hat{z}_{n_{j_{l}}}^{*}-y^{*}\right)\left(y_{n_{j_{s}}}\right) \rightarrow b \quad \text { para algum } b \in \mathbb{K}
$$

Para os índices $n_{j_{l_{s}}}$ 's, a sequência $\left(\left(\hat{z}_{n_{j_{l_{s}}}}-y^{*}\right)\left(x_{n_{j_{l_{s}}}}\right)\right)_{s}$ é uma subsequência de $\left(\left(\stackrel{\wedge}{z}_{n_{j_{l}}}-y^{*}\right)\left(x_{n_{j_{l}}}\right)\right)_{l}, \operatorname{logo}$

$$
\left(\hat{z}_{n_{j_{l}}}^{*}-y^{*}\right)\left(x_{n_{j_{l}}}\right) \rightarrow a
$$

Afirmamos que $a \neq b$, pois

$$
\begin{gathered}
a-b=\lim _{s \rightarrow \infty}\left[\left(\hat{z}_{n_{j_{l_{s}}}}^{*}-y^{*}\right)\left(x_{n_{j_{l_{s}}}}\right)-\left(\hat{z}_{n_{j_{l_{s}}}}^{*}-y^{*}\right)\left(y_{n_{j_{l_{s}}}}\right)\right] \\
=\lim _{s \rightarrow \infty}\left(\stackrel{\wedge}{z}_{n_{j_{l_{s}}}^{*}}\right)\left(z_{n_{j_{l_{s}}}}\right)=1,
\end{gathered}
$$

por 3.9. Assim, para cada número natural $q$ impar temos que $\left|a^{q}-b^{q}\right|>0$. Desta maneira, escolhendo um $q>p$ impar, temos que $\left|a^{q}-b^{q}\right|=R>0, l_{p} \subset l_{q}$ e $\|\cdot\|_{q} \leq\|\cdot\|_{p}$ logo,

$$
\sum_{s=1}^{\infty}\left|\left(\stackrel{\wedge}{z}_{n_{j_{l}}}^{*}-y^{*}\right)(x)\right|^{q} \leq \sum_{j=1}^{\infty}\left|\left(\hat{z}_{n_{j}}^{*}-y^{*}\right)(x)\right|^{q} \leq \sum_{j=1}^{\infty}\left|\left(\hat{z}_{n_{j}}^{*}-y^{*}\right)(x)\right|^{p}<\infty
$$

ou seja, a série é absolutamente convergente para cada $x \in X$.

Assim, definindo $P: X \longrightarrow \mathbb{K}$ por:

$$
P(x)=\sum_{s=1}^{\infty}\left[\left(\hat{z}_{n_{l_{l}}}^{*}-y^{*}\right)(x)\right]^{q} \quad \text { para cada } x \in X
$$


afirmamos que $P \in \mathcal{P}\left({ }^{q} X\right)$. De fato: para cada $k \in \mathbb{N}$, seja $P_{k}: X \longrightarrow \mathbb{K}$ dado por:

$$
P_{k}(x)=\sum_{s=1}^{k}\left[\left(\hat{z}_{n_{j_{l s}}}^{*}-y^{*}\right)(x)\right]^{q} \quad \text { para cada } x \in X \text {. }
$$

Temos que $P_{k} \in \mathcal{P}\left({ }^{q} X\right)$ para cada $k \in \mathbb{N}$ e para cada $x \in X, P_{k}(x) \stackrel{k \rightarrow \infty}{\longrightarrow} P(x)$. Logo, pelo teorema de Banach-Steinhaus para polinômios (teorema 2.4.8) temos que $P \in \mathcal{P}\left({ }^{q} X\right)$. Para cada $t \in \mathbb{N}$, temos:

$$
\begin{aligned}
& \left|P\left(x_{n_{j_{l_{t}}}}\right)-P\left(y_{n_{l_{l_{t}}}}\right)\right|=\left|\sum_{s=1}^{\infty}\left[\left(\hat{z}_{n_{j_{l_{s}}}^{*}}^{*}-y^{*}\right)\left(x_{n_{j_{l_{t}}}}\right)\right]^{q}-\sum_{s=1}^{\infty}\left[\left(\hat{z}_{n_{j_{l_{s}}}}^{*}-y^{*}\right)\left(y_{n_{j_{l_{t}}}}\right)\right]^{q}\right| \\
& =\mid \sum_{s=1}^{t-1}\left[\left[\left(\hat{z}_{n_{j_{l_{s}}}}^{*}-y^{*}\right)\left(x_{n_{j_{l_{t}}}}\right)\right]^{q}-\left[\left(\hat{z}_{n_{j_{l_{s}}}}^{*}-y^{*}\right)\left(y_{n_{j_{l_{t}}}}\right)\right]^{q}\right]+ \\
& \sum_{s=t+1}^{\infty}\left[\left[\left(\hat{z}_{n_{j_{l_{s}}}}^{*}-y^{*}\right)\left(x_{n_{j_{l_{t}}}}\right)\right]^{q}-\left[\left(\hat{z}_{n_{j_{l_{s}}}^{*}}-y^{*}\right)\left(y_{n_{j_{l_{t}}}}\right)\right]^{q}\right]+ \\
& {\left[\left[\left(\hat{z}_{n_{j_{l_{t}}}}^{*}-y^{*}\right)\left(x_{n_{j_{l_{t}}}}\right)\right]^{q}-\left[\left(\hat{z}_{n_{j_{l_{t}}}^{*}}-y^{*}\right)\left(y_{n_{j_{l_{t}}}}\right)\right]^{q} \mid\right.} \\
& =\left|\left[\left(\hat{z}_{n_{j_{l_{t}}}}^{*}-y^{*}\right)\left(x_{n_{j_{l_{t}}}}\right)\right]^{q}-\left[\left(\hat{z}_{n_{j_{l_{t}}}^{*}}-y^{*}\right)\left(y_{n_{j_{l_{t}}}}\right)\right]^{q}\right| \text {. }
\end{aligned}
$$

Logo, por 3.9, 3.10 e 3.11 temos que

$$
\left|P\left(x_{n_{j_{t}}}\right)-P\left(y_{n_{j_{t}}}\right) \stackrel{t \rightarrow \infty}{\longrightarrow}\right| a^{q}-b^{q} \mid=R>0,
$$

que contradiz a hipótese inicial. Portanto $\left\|x_{n}-y_{n}\right\| \stackrel{n \rightarrow \infty}{\longrightarrow} 0$, para as sequências iniciais $\left(x_{n}\right)_{n}$ e $\left(y_{n}\right)_{n}$, ou seja, $X$ tem a propriedade $(\star \star)$. Logo pelo teorema 3.2.4 $X$ tem a propriedade $\mathbf{P}$.

Corolário 3.2.12. Seja $X$ um espaço de Banach de dimensão infinita. Se $X$ é superreflexivo então $X$ tem a propriedade $(\star \star)$, e consequentemente a propriedade $\mathbf{P}$.

Demonstração: Seja $X$ um espaço super-reflexivo de dimensão infinita. Pelo teorema 1.3.4 temos que $X^{*} \in W_{p}$ para algum $p>1$. Logo, pelo teorema 3.2.11, temos que $X$ tem a propriedade $(\star \star)$, e consequentemente, $X$ tem a propriedade $\mathbf{P}$.

Exemplo 3.2.13. Os espaços $l_{p}$ e $L_{p}[0,1], 1<p<\infty$, são super-reflexivos (ver exemplo 1.3.9), logo, pelo corolário acima, eles têm a propriedade ( $\star \star)$ e consequentemente, têm a propriedade $\mathbf{P}$. 
No próximo teorema vamos mostrar que a propriedade $\mathbf{P}$ é preservada por isomorfismos.

Teorema 3.2.14. Sejam $X, Y$ espaço de Banach. Se $X$ tem a propriedade $\mathbf{P}$ e $Y$ é isomorfo a $X$ então $Y$ tem a propriedade $\mathbf{P}$.

Demonstração: Sejam $\left(u_{j}\right)_{j}$ e $\left(v_{j}\right)_{j}$ sequências limitadas em $Y$ tais que para cada $m \geq 1$ e cada $P \in \mathcal{P}\left({ }^{m} Y\right)$ temos que $\left|P\left(u_{j}\right)-P\left(v_{j}\right)\right| \stackrel{j \rightarrow \infty}{\longrightarrow} 0$. Como $Y$ é isomorfo a $X$, existe $T: Y \longrightarrow X$ um isomorfismo. Assim, existem $\left(x_{j}\right)_{j}$ e $\left(y_{j}\right)_{j}$ sequências limitadas em $X$ tais que

$$
\left(T\left(u_{j}\right)\right)_{j}=\left(x_{j}\right)_{j} \quad \text { e } \quad\left(T\left(v_{j}\right)\right)_{j}=\left(y_{j}\right)_{j}
$$

Agora, para cada $m \geq 1$ e cada $Q^{*} \in \mathcal{P}\left({ }^{m} X\right)$, pelo teorema 2.4.6, temos que $Q^{*} \circ T \in \mathcal{P}\left({ }^{m} Y\right)$, logo, por hipótese, $\left|Q^{*} \circ T\left(u_{j}\right)-Q^{*} \circ T\left(v_{j}\right)\right| \stackrel{j \rightarrow \infty}{\longrightarrow} 0$. Como $X$ tem a propriedade $\mathbf{P}$ então $\left|Q\left(x_{j}-y_{j}\right)\right| \stackrel{j \rightarrow \infty}{\longrightarrow} 0$ para cada $Q \in \mathcal{P}\left({ }^{n} X\right)$ e $n \geq 1$.

Sejam $N \in \mathbb{N}$ e $P^{*} \in \mathcal{P}\left({ }^{N} Y\right)$. Vamos provar que $\mid P^{*}\left(u_{j}-v_{j}\right) \stackrel{j \rightarrow \infty}{\longrightarrow} 0$. Para isso, defina$\operatorname{mos} R: X \longrightarrow \mathbb{K}$ por $R(x)=\left(P^{*} \circ T^{-1}\right)(x)$ para cada $x \in X$. Assim, pelo teorema 2.4.6, temos que $R \in \mathcal{P}\left({ }^{N} X\right)$, e consequentemente, temos que $\left|R\left(x_{j}-y_{j}\right)\right| \stackrel{j \rightarrow \infty}{\longrightarrow} 0$, ou seja, $\left|\left(P^{*} \circ T^{-1}\right)\left(x_{j}-y_{j}\right)\right| \stackrel{j \rightarrow \infty}{\longrightarrow} 0$. Mas para cada $j \in \mathbb{N},\left|\left(P^{*} \circ T^{-1}\right)\left(x_{j}-y_{j}\right)\right|=\left|P^{*}\left(u_{j}-v_{j}\right)\right|$. Logo, $\left|P^{*}\left(u_{j}-v_{j}\right)\right| \stackrel{j \rightarrow \infty}{\longrightarrow} 0$. Portanto $Y$ tem a propriedade $\mathbf{P}$.

$\mathrm{Na}$ demonstração de que espaços de Banach com a propriedade Dunford-Pettis tem a propriedade $\mathbf{P}$ usamos fortemente a proposição 3.1.7(1). A seguir vamos estudar o fato de que espaços com propriedades mais fracas que a Dunford-Pettis têm a propriedade $\mathbf{P}$, dentre elas, são os espaços onde cada polinômio é fracamente sequencialmente contínuo em um determinado ponto.

Teorema 3.2.15. Seja $X$ um espaço de Banach. Suponhamos que todo $P: X \longrightarrow \mathbb{K} e ́$ fracamente sequencialmente contínuo na origem. Então $X$ tem a propriedade $\mathrm{P}$.

Demonstração: Sejam $\left(u_{j}\right)_{j},\left(v_{j}\right)_{j}$ sequências limitadas em $X$ tais que para cada $m \geq 1$ e cada $P \in \mathcal{P}\left({ }^{m} X\right)$ temos que $\mid P\left(u_{j}\right)-P\left(v_{j}\right) \stackrel{j \rightarrow \infty}{\longrightarrow} 0, \operatorname{logo} u_{j}-v_{j} \stackrel{\omega}{\rightarrow} 0$.

Sejam $n \in \mathbb{N}$, e $Q \in \mathcal{P}\left({ }^{n} X\right)$, como $Q$ é fracamente sequencialmente contínuo na origem então $\left|Q\left(u_{j}-v_{j}\right)\right| \stackrel{j \rightarrow \infty}{\longrightarrow} 0$. Portanto $X$ tem a propriedade $\mathbf{P}$. 
Por outro lado, o espaço $l_{2}$ tem a propriedade $\mathbf{P}$ e o polinômio 3-homogêneo $Q$ dado por:

$$
Q\left(\left(x_{n}\right)_{n}\right)=\sum_{n=1}^{\infty} x_{n}^{3}
$$

com $\left(x_{n}\right)_{n} \in l_{2}$, não é fracamente sequencialmente contínuo na origem, uma vez que para cada $j \in \mathbb{N}, e_{j}$ é o j-ésimo elemento da base canônica de $l_{2}$, temos que $e_{j} \stackrel{\omega}{\rightarrow} 0$, mas $\left|Q\left(e_{j}\right)\right|=1>0$ para cada $j \in \mathbb{N}$.

Do teorema, segue imediatamente:

Corolário 3.2.16. Seja $X$ um espaço de Banach. Suponhamos que $\mathcal{P}(X)=\mathcal{P}_{\text {wsc }}(X)$ então $X$ tem propriedade $\mathbf{P}$.

A seguir apresentamos mais um exemplo de um espaço que tem a propriedade $\mathbf{P}$ e não tem a Dunford-Pettis.

Em [31], Tsirelson descreveu um método de como construir uma classe de espaços com propriedades especiais. Não descrevemos aqui a construção de tais espaços, mas sim as propriedades que o espaço original de Tsirelson $T^{*}$ possui. O espaço $T^{*}$ é um espaço de Banach reflexivo de dimensão infinita, que não contém subespaços isomorfos a $c_{0}$ ou quaisquer $l_{p}(1 \leq p<\infty)$.

Exemplo 3.2.17. O espaço original de Tsirelson $T^{*}$ tem a propriedade $\mathbf{P}$, não tem Dunford-Pettis e não tem a propriedade ( $\star \star)$.

De fato: Alencar, Aron \& Dineen em [1] provaram que cada polinômio contínuo sobre o espaço original de Tsirelson $T^{*}$ é fracamente sequencialmente contínuo, logo, pelo corolário 3.2.16, $T^{*}$ tem a propriedade $\mathbf{P}$. Agora $T^{*}$ não tem a propriedade Dunford-Pettis, pois não satisfaz a condição 1 da proposição 3.1.4.

Jaramillo \& Prieto em [[23], proposição 1], mostraram que o espaço $T^{*}$ não é polinomialmente Schur, logo, pelo teorema $3.2 .4, T^{*}$ não tem a propriedade $(\star \star)$.

A seguir, o nosso objetivo é estudar resultados que garantem que os espaços de tipo não-trivial (definido no capítulo 1 , seção 3) têm a propriedade $\mathbf{P}$.

O próximo teorema foi demonstrado por Biström, Jaramillo \& Lindström em [8]. 
Teorema 3.2.18. Seja $X$ um espaço de Banach tal que $l_{1} \leftrightarrow X^{*}$ e $X^{*}$ tem a propriedade $S_{p}$ para algum $p, 1<p<\infty$. Então $X$ tem a propriedade $(\star \star)$, e consequentemente, $X$ tem a propriedade $\mathbf{P}$.

Demonstração: Sejam $\left(x_{n}\right)_{n},\left(y_{n}\right)_{n}$ sequências limitadas em $X$ tais que para cada $P \in \mathcal{P}(X)$ temos que $\left|P\left(x_{n}\right)-P\left(y_{n}\right)\right| \stackrel{n \rightarrow \infty}{\longrightarrow} 0$. Suponhamos que $\left\|x_{n}-y_{n}\right\| \nrightarrow \rightarrow 0$, então existem $\epsilon>0$ e subsequências de $\left(x_{n}\right)_{n}$ e $\left(y_{n}\right)_{n}$, que vamos denotar por $\left(x_{n}\right)_{n}$ e $\left(y_{n}\right)_{n}$ respectivamente, tais que $\left\|x_{n}-y_{n}\right\|>\epsilon$ para cada $n \in \mathbb{N}$. Como a subsequência $x_{n}-y_{n} \stackrel{\omega}{\longrightarrow} 0$, pelo teorema de Bessaga-Pelczynski (1.2.32), $\left(x_{n}-y_{n}\right)_{n}$ admite uma subsequência básica, que vamos denotar por $\left(x_{n}-y_{n}\right)_{n}$, a qual será usada daqui em diante nesta demonstração. Para cada $n \in \mathbb{N}$, seja $z_{n}=x_{n}-y_{n}, \operatorname{logo}\left\|z_{n}\right\| \geq \epsilon$ e $z_{n} \stackrel{\omega}{\longrightarrow} 0$. Sejam $Z$ o subespaço fechado de $X$ gerado por $\left(z_{n}\right)_{n}$ e $\left(z_{n}^{*}\right)_{n}$ a sequência de funcionais associados a $\left(z_{n}\right)_{n}$. Pelo teorema 1.2.14, para cada $n \in \mathbb{N}, z_{n}^{*}$ é limitado em $Z$, logo pelo teorema de Hahn-Banach (1.2.6) existe $\hat{z}_{n}^{*} \in X^{*}$ tal que $\left\|\hat{z}_{n}^{*}\right\|=\left\|z_{n}^{*}\right\|$. Assim, pelo teorema 1.2.14 $\left(z_{n}^{*}\right)_{n}$ é limitada em $X^{*}$. Como $l_{1} \nrightarrow X^{*}$ então pelo teorema de Rosenthal (1.2.33), $\left(\hat{z}_{n}^{*}\right)_{n}$ admite uma subsequência, que vamos denotar por $\left(\hat{z}_{n_{j}}^{*}\right)_{j}$, que é fracamente Cauchy. Assim, para cada $j \in \mathbb{N}$ considere $\varphi_{j}:=\hat{\wedge}_{n_{2 j+1}^{*}}^{*}-\hat{\varkappa}_{n_{2 j}}^{*}$, então $\varphi_{j} \stackrel{\omega}{\longrightarrow} 0$, ou seja, para cada $\psi \in X^{* *}$ temos que

$$
\left|\psi\left(\varphi_{j}\right)\right|=\left|\psi\left(\hat{z}_{n_{2 j+1}}^{*}\right)-\psi\left(\hat{z}_{n_{2 j}}^{*}\right)\right| \stackrel{j \rightarrow \infty}{\longrightarrow} 0
$$

pois $\left(\hat{z}_{n_{j}}^{*}\right)_{j}$ é fracamente Cauchy. Definamos $\left(w_{k}\right)_{k}$ uma subsequência de $\left(z_{n_{j}}\right)_{j}$ da seguinte maneira: para cada $k \in \mathbb{N}, w_{k}=z_{n_{2 k+1}}$ então

$$
\varphi_{j}\left(w_{k}\right)=\left(\hat{z}_{n_{2 j+1}}^{*}-\hat{z}_{n_{2 j}}^{*}\right)\left(z_{n_{2 k+1}}\right)=\hat{z}_{n_{2 j+1}}^{*}\left(z_{n_{2 k+1}}\right)-{\stackrel{\wedge}{z_{n}}}_{n_{2 j}}^{*}\left(z_{n_{2 k+1}}\right)=\delta_{j k}-0=\delta_{j k} .
$$

Como $X^{*}$ tem a propriedade $S_{p}$, existe uma subsequência $\left(\varphi_{j_{l}}\right)_{l}$ de $\left(\varphi_{j}\right)_{j}$ tal que $\left(\psi\left(\varphi_{j_{l}}\right)\right)_{l} \in l_{q}$ para cada $\psi \in X^{* *}$ onde $\frac{1}{p}+\frac{1}{q}=1$.

Para cada $x \in X$, considere a função $J_{x} \in X^{* *}$ dado por: $J_{x}\left(y^{*}\right)=y^{*}(x)$.

Assim, para cada $x \in X$,

$$
\sum_{l=1}^{\infty}\left|\varphi_{j_{l}}(x)\right|^{q}=\sum_{l=1}^{\infty}\left|J_{x}\left(\varphi_{j_{l}}\right)\right|^{q}<\infty .
$$

Definindo uma aplicação linear contínua $T: X \longrightarrow l_{q}$ por:

$$
T(x)=\left(J_{x}\left(\varphi_{j_{l}}\right)\right)_{l}
$$

temos que para cada $k \in \mathbb{N}$,

$$
T\left(w_{k}\right)=\left(J_{w_{k}}\left(\varphi_{j_{l}}\right)\right)_{l}=\left(\varphi_{j_{l}}\left(w_{k}\right)\right)_{l}=\left(\delta_{j_{l} k}\right)_{l}=e_{k}
$$


onde $e_{k}$ é o k-ésimo elemento da base canônica de $l_{q}$. Para cada $m \in \mathbb{N}$, seja $P \in \mathcal{P}\left({ }^{m} l_{q}\right)$ então, pelo teorema 2.4.6, $P \circ T \in \mathcal{P}\left({ }^{m} X\right)$, pois T é linear contínua. Assim, por hipótese, temos que $\left|P \circ T\left(x_{n}\right)-P \circ T\left(y_{n}\right)\right| \rightarrow 0$, ou seja,

$$
\left|P\left(T\left(x_{n}\right)\right)-P\left(T\left(y_{n}\right)\right)\right| \stackrel{n \rightarrow \infty}{\longrightarrow} 0 .
$$

As sequências $\left(T\left(x_{n}\right)\right)_{n},\left(T\left(y_{n}\right)\right)_{n}$ são limitadas em $l_{q}$. Como o espaço $l_{q}$ tem a propriedade $(\star \star)$ temos que $\left\|T\left(x_{n}\right)-T\left(y_{n}\right)\right\|_{q} \stackrel{n \rightarrow \infty}{\longrightarrow} 0$. Mas, para cada $n \in \mathbb{N}$,

$\left\|T\left(x_{n}\right)-T\left(y_{n}\right)\right\|_{q}=\left\|T\left(z_{n}\right)\right\|_{q}$, logo para qualquer subsequência de $\left(T\left(z_{n}\right)\right)_{n}$ convergente em norma teríamos que converge a zero em norma, em particular, $\left(T\left(w_{k}\right)\right)_{k}$ converge a zero em norma. Desta maneira, $\left\|e_{k}\right\| \stackrel{k \rightarrow \infty}{\longrightarrow} 0$, contradição, pois $\left\|e_{k}\right\|=1$ para cada $k \in \mathbb{N}$.

Logo $\left\|x_{n}-y_{n}\right\| \stackrel{n \rightarrow \infty}{\longrightarrow} 0$ para as sequências $\left(x_{n}\right)_{n}$ e $\left(y_{n}\right)_{n}$ iniciais, ou seja, $X$ tem a propriedade ( $\star \star)$. Portanto, pelo teorema 3.2.4, temos que $X$ tem a propriedade $\mathbf{P}$.

Observamos que se um espaço de Banach $X$ é super-reflexivo então ele satisfaz as condições do teorema acima, pois $X^{*} \in S_{p}$ para algum $1<p<\infty$ (ver por exemplo [10]) e pelo teorema de Eberlein-Smulian (1.2.24) temos que cada sequência $\left(x_{n}^{*}\right)_{n} \subset X^{*}$ limitada admite uma subsequência $\left(x_{n_{j}}^{*}\right)_{j}$ fracamente Cauchy, logo, pelo teorema de Rosenthal $(1.2 .33), l_{1} \hookrightarrow X^{*}$.

Farmer \& Jonhson em [16] provaram que todo espaço de Banach com tipo não-trivial é polinomialmente Schur. Agora, no resultado que segue, tais estes espaços têm a propriedade $\mathbf{P}$. Lembramos que um espaço de Banach é de tipo não-trivial se ele é de tipo p, para algum $p>1$ (que se encontra definido no capítulo 1, seção 3 ).

Usando o resultado provado por Gonzalo \& Jaramillo em [19] que nos diz que se $X$ tem tipo $p, p \in(1,2]$, então $X \in S_{r}$ para todo $r<p$, temos o seguinte corolário:

Corolário 3.2.19. Seja $X$ um espaço de Banach. Se $X$ é de tipo não-trivial então $X$ tem a propriedade $(\star \star)$, e consequentemente, $X$ tem a propriedade $\mathbf{P}$.

Demonstração: Seja $X$ um espaço de Banach de tipo não-trivial, então pelo teorema 1.3.12 temos que $X^{*} \in S_{r}$ para todo $r<p$. Se $l_{1} \hookrightarrow X^{*}$ então $l_{1}$ tería tipo $p>1$, absurdo, pois $l_{1}$ não tem tipo não-trivial (ver o exemplo 1.3.11). Assim, pelo teorema 3.2.18, $X$ tem a propriedade $(\star \star)$ e consequentemente, $X$ tem a propriedade $\mathbf{P}$.

No próximo teorema vamos estudar a propriedade $\mathbf{P}$ no espaço $X \times Y$, onde $X$ tem a propriedade Dunford-Pettis e $Y$ tem a propriedade $\mathbf{P}$. A demonstração encontra-se em [8]. 
Teorema 3.2.20. Sejam $X, Y$ espaços de Banach tais que $X$ tem a propriedade DunfordPettis e $Y$ tem a propriedade $\mathbf{P}$, então $X \times Y$ tem a propriedade $\mathbf{P}$.

Demonstração: No espaço $X \times Y$ vamos considerar o norma dada pelo sup. Sejam $\left(x_{n}, y_{n}\right)_{n}$ e $\left(z_{n}, w_{n}\right)_{n}$ sequências limitadas em $X \times Y$ tais que para cada $l \in \mathbb{N}$ e cada $P \in \mathcal{P}\left({ }^{l} X \times Y\right)$ temos que $\left|P\left(\left(x_{n}, y_{n}\right)\right)-P\left(\left(z_{n}, w_{n}\right)\right)\right| \stackrel{n \rightarrow \infty}{\longrightarrow} 0$. Vamos mostrar que $\left|S\left(\left(x_{n}, y_{n}\right)-\left(z_{n}, w_{n}\right)\right)\right| \stackrel{n \rightarrow \infty}{\longrightarrow} 0$ para cada $S \in \mathcal{P}(r(X \times Y))$ e cada $r \in \mathbb{N}$. Primeiramente, vamos mostrar que :

1. $\left|Q\left(x_{n}-z_{n}\right)\right| \stackrel{n \rightarrow \infty}{\longrightarrow} 0$ para cada $Q \in \mathcal{P}\left({ }^{m} X\right)$ e $m \geq 1$.

2. $\left|R\left(y_{n}-w_{n}\right)\right| \rightarrow 0$ para cada $R \in \mathcal{P}\left({ }^{m} Y\right)$ e $m \geq 1$.

Sejam $k \in \mathbb{N}$ e $Q_{1} \in \mathcal{P}\left({ }^{k} X\right)$. Definamos $P_{1}: X \times Y \longrightarrow \mathbb{K}$ por:

$$
P_{1}(x, y)=\left(Q_{1} \circ \Pi_{1}\right)(x, y) \quad \text { para cada }(x, y) \in X \times Y
$$

onde $\Pi_{1}$ é a aplicação projeção de $X \times Y$ em X dada por $\Pi_{1}((x, y))=x$.

Então, pelo teorema 2.4.6, $P_{1} \in \mathcal{P}\left({ }^{k} X \times Y\right)$ e por hipótese, $\left|P_{1}\left(\left(x_{n}, y_{n}\right)\right)-P_{1}\left(\left(z_{n}, w_{n}\right)\right)\right| \stackrel{n \rightarrow \infty}{\longrightarrow} 0$. Mas, para cada $n \in \mathbb{N}$, temos que:

$$
\begin{aligned}
\left|P_{1}\left(\left(x_{n}, y_{n}\right)\right)-P_{1}\left(\left(z_{n}, w_{n}\right)\right)\right| & =\left|\left(Q_{1} \circ \Pi_{1}\right)\left(\left(x_{n}, y_{n}\right)\right)-\left(Q_{1} \circ \Pi_{1}\right)\left(\left(z_{n}, w_{n}\right)\right)\right| \\
& =\left|Q_{1}\left(x_{n}\right)-Q_{1}\left(z_{n}\right)\right|
\end{aligned}
$$

e assim $\left|Q_{1}\left(x_{n}\right)-Q_{1}\left(z_{n}\right)\right| \stackrel{n \rightarrow \infty}{\longrightarrow} 0$. Como $X$ tem a propriedade Dunford-Pettis segue do teorema 3.2.4 que $X$ tem a propriedade $\mathbf{P}$ então

$$
\left|Q\left(x_{n}-z_{n}\right)\right| \stackrel{n \rightarrow \infty}{\longrightarrow 0} \text { para cada } Q \in \mathcal{P}\left({ }^{m} X\right) \quad \text { e } \quad m \geq 1 .
$$

Analogamente, para cada $k \in \mathbb{N}$ e $R_{2} \in \mathcal{P}\left({ }^{k} Y\right)$, definindo $P_{2}: X \times Y \longrightarrow \mathbb{K}$ por:

$$
P_{2}(x, y)=\left(R_{2} \circ \Pi_{2}\right)(x, y) \quad \text { para cada }(x, y) \in X \times Y
$$

onde $\Pi_{2}$ é a aplicação projeção de $X \times Y$ em Y dada por $\Pi_{2}((x, y))=y$ temos que $\left|R_{2}\left(y_{n}\right)-R_{2}\left(w_{n}\right)\right| \stackrel{n \rightarrow \infty}{\longrightarrow} 0$ e como $Y$ tem a propriedade $\mathbf{P}$ então

$$
\left|R\left(y_{n}-w_{n}\right)\right| \stackrel{n \rightarrow \infty}{\longrightarrow} 0 \quad \text { para cada } \quad R \in \mathcal{P}\left({ }^{m} Y\right) \quad \text { e } \quad m \geq 1 .
$$

Sejam $r \in \mathbb{N}$ e $S \in \mathcal{P}\left({ }^{r}(X \times Y)\right)$. Seja $A$ a aplicação r-linear contínua associada ao polinômio $S$.

$$
\begin{aligned}
& S\left(\left(x_{n}, y_{n}\right)-\left(z_{n}, w_{n}\right)\right)=A\left(\left(x_{n}, y_{n}\right)-\left(z_{n}, w_{n}\right), \stackrel{(r)}{\cdot},\left(x_{n}, y_{n}\right)-\left(z_{n}, w_{n}\right)\right) \\
& =A\left(\left(x_{n}-z_{n}, 0\right), \stackrel{(r)}{.},\left(x_{n}-z_{n}, 0\right)\right)+A\left(\left(0, y_{n}-w_{n}\right), \stackrel{(r)}{.},\left(0, y_{n}-w_{n}\right)\right)
\end{aligned}
$$




$$
\begin{aligned}
& +\sum_{j=1}^{r-1} A\left(\left(x_{n}-z_{n}, 0\right), \stackrel{(j)}{\cdot},\left(x_{n}-z_{n}, 0\right),\left(0, y_{n}-w_{n}\right), \stackrel{(r-j)}{\cdots},\left(0, y_{n}-w_{n}\right)\right) \\
& =S\left(\left(x_{n}-z_{n}, 0\right)\right)+S\left(\left(0, y_{n}-w_{n}\right)\right)+ \\
& \sum_{j=1}^{r-1} A\left(\left(x_{n}-z_{n}, 0\right), \stackrel{(j)}{\cdot} \cdot,\left(x_{n}-z_{n}, 0\right),\left(0, y_{n}-w_{n}\right), \stackrel{(r-j)}{\cdot},\left(0, y_{n}-w_{n}\right)\right)
\end{aligned}
$$

Agora vamos mostrar que cada componente da soma acima converge a zero quando $n \rightarrow \infty$. Primeiramente, vamos definir o polinômio $Q^{*}: X \longrightarrow \mathbb{K}$ por:

$$
Q^{*}(x)=S(x, 0) \quad \text { para cada } x \in X,
$$

então $Q^{*} \in \mathcal{P}\left({ }^{r} X\right)$. Assim, de 3.12 temos que $\left|Q^{*}\left(x_{n}-z_{n}\right)\right| \stackrel{n \rightarrow \infty}{\rightarrow} 0$. Mas, para cada $n \in \mathbb{N}, Q^{*}\left(x_{n}-z_{n}\right)=S\left(\left(x_{n}-z_{n}, 0\right)\right), \log \mathrm{o}$,

$$
\left|S\left(\left(x_{n}-z_{n}, 0\right)\right)\right| \stackrel{n \rightarrow \infty}{\longrightarrow} 0 .
$$

Com argumentos análogos, definindo $R^{*}: Y \longrightarrow \mathbb{K}$ por:

$$
R^{*}(y)=S(0, y) \quad \text { para cada } x \in X
$$

temos que

$$
\left|S\left(\left(0, y_{n}-w_{n}\right)\right)\right| \stackrel{n \rightarrow \infty}{\longrightarrow} 0 .
$$

Para as demais componentes da soma, para cada $j \in\{1, \ldots, r-1\}$ vamos definir uma sequência $\left(P_{n}^{[j]}\right)_{n} \in \mathcal{P}(X)$ tal que $P_{n}^{[j]} \stackrel{\omega}{\rightarrow} 0$.

Para cada $j \in\{1, \ldots, r-1\}$, seja $\left(P_{n}^{[j]}\right)_{n}$ a sequência dada da seguinte maneira:

Para cada $n \in \mathbb{N}, P_{n}^{[j]}: X \longrightarrow \mathbb{K}$ é dado por:

$$
P_{n}^{[j]}(x)=A\left((x, 0), \stackrel{(j)}{\cdot},(x, 0),\left(0, y_{n}-w_{n}\right), \stackrel{(r-j)}{\cdots},\left(0, y_{n}-w_{n}\right)\right)
$$

para cada $x \in X$. Assim, para cada $n \in \mathbb{N}, P_{n}^{[j]}$ é um polinômio j-homogêneo contínuo. Vamos mostrar que $P_{n}^{[j]} \stackrel{\omega}{\rightarrow} 0$ quando $n \rightarrow \infty$. Seja $\phi \in \mathcal{P}\left({ }^{j} X\right)^{*}$. Definamos $Q^{[j]}: Y \longrightarrow \mathcal{P}\left({ }^{j} X\right)$ por:

Para cada $y \in Y$ e cada $x \in X$

$$
Q^{[j]}(y)(x)=A((x, 0), \stackrel{(j)}{\cdot},(x, 0),(0, y), \stackrel{(r-j)}{\cdots},(0, y))
$$

Assim, $Q^{[j]}$ é um polinômio ( $\mathrm{r}$ - j)-homogêneo contínuo, logo $\phi \circ Q^{[j]} \in \mathcal{P}\left({ }^{r-j} Y\right)$.

Como $Y$ tem a propriedade $\mathbf{P}$, então por 3.13 temos que $\left|\left(\phi \circ Q^{[j]}\right)\left(y_{n}-w_{n}\right)\right| \stackrel{n \rightarrow \infty}{\longrightarrow} 0$. Mas

$$
\begin{gathered}
\left(\phi \circ Q^{[j]}\right)\left(y_{n}-w_{n}\right)=\phi\left(Q^{[j]}\left(y_{n}-w_{n}\right)\right)= \\
\phi\left(A\left((\cdot, 0), \stackrel{(j)}{\cdot},(\cdot, 0),\left(0, y_{n}-w_{n}\right),{ }^{(r-j)},\left(0, y_{n}-w_{n}\right)\right)\right)=\phi\left(P_{n}^{[j]}\right) .
\end{gathered}
$$


Assim, $\left|\phi\left(P_{n}^{[j]}\right)\right| \stackrel{n \rightarrow \infty}{\longrightarrow} 0$, ou seja, $P_{n}^{[j]} \stackrel{\omega}{\rightarrow} 0$.

Como $X$ tem a propriedade Dunford-Pettis e vale 3.12, então, pelo teorema 3.1.7(2), $\left|P_{n}^{[j]}\left(x_{n}-z_{n}\right)\right| \stackrel{n \rightarrow \infty}{\longrightarrow} 0$.

Logo, para cada $j \in\{1, \ldots, r-1\}$

$$
\left|A\left(\left(x_{n}-z_{n}, 0\right), \stackrel{(j)}{.},\left(x_{n}-z_{n}, 0\right),\left(0, y_{n}-w_{n}\right), \stackrel{(r-j)}{\cdots},\left(0, y_{n}-w_{n}\right)\right)\right| \stackrel{n \rightarrow \infty}{\longrightarrow} 0 .
$$

Assim, dado $\epsilon>0$ temos: de 3.14 existe $m_{0} \in \mathbb{N}$ tal que $\left|S\left(\left(x_{n}-z_{n}, 0\right)\right)\right|<\frac{\epsilon}{3}$ para todo $n \geq m_{0}$. De 3.15 existe $m_{1} \in \mathbb{N}$ tal que $\left|S\left(\left(0, y_{n}-w_{n}\right)\right)\right|<\frac{\epsilon}{3}$ para todo $n \geq m_{1}$. De 3.16 , para cada $j \in\{1, \ldots, r-1\}$ existe $n_{j} \in \mathbb{N}$ tal que

$$
\left|A\left(\left(x_{n}-z_{n}, 0\right), \stackrel{(j)}{\cdot},\left(x_{n}-z_{n}, 0\right),\left(0, y_{n}-w_{n}\right), \stackrel{(r-j)}{\cdots},\left(0, y_{n}-w_{n}\right)\right)\right|<\frac{\epsilon}{3(r-1)}
$$

para todo $n \geq n_{j}$. Mas para cada $n \in \mathbb{N}$,

$$
\begin{gathered}
\left|S\left(\left(x_{n}, y_{n}\right)-\left(z_{n}, w_{n}\right)\right)\right| \leq\left|S\left(\left(x_{n}-z_{n}, 0\right)\right)\right|+\left|S\left(\left(0, y_{n}-w_{n}\right)\right)\right|+ \\
\sum_{j=1}^{r-1}\left|A\left(\left(x_{n}-z_{n}, 0\right), \stackrel{(j)}{.},\left(x_{n}-z_{n}, 0\right),\left(0, y_{n}-w_{n}\right), \stackrel{(r-j)}{\cdot},\left(0, y_{n}-w_{n}\right)\right)\right|
\end{gathered}
$$

Agora, tomando $n_{0}=\sup \left\{m_{0}, m_{1}, n_{1}, \ldots, n_{r-1}\right\}$, temos que para cada $n \geq n_{0}$,

$$
\left|S\left(\left(x_{n}, y_{n}\right)-\left(z_{n}, w_{n}\right)\right)\right| \leq \frac{\epsilon}{3}+\frac{\epsilon}{3}+(r-1) \frac{\epsilon}{3(r-1)}=\epsilon
$$

$\operatorname{logo}\left|S\left(\left(x_{n}, y_{n}\right)-\left(z_{n}, w_{n}\right)\right)\right| \stackrel{n \rightarrow \infty}{\longrightarrow} 0$. Portanto $X \times Y$ tem a propriedade $\mathbf{P}$.

Aron, Choi \& Llavona em [2] colocaram a seguinte pergunta:

Existe um espaço de Banach que não tem a propriedade $\mathbf{P}$ ?

Choi \& Kim em [12] conseguiram encontrar condições suficientes para que um espaço não tenha a propriedade $\mathbf{P}$. Tais condições são dadas no seguinte teorema:

Teorema 3.2.21. Seja X um espaço de Banach de dimensão infinita. Se $X$ é polinomialmente Schur e se para cada $n \in \mathbb{N}, \mathcal{P}\left({ }^{n} X\right)$ é separável então $X$ não tem a propriedade P.

Demonstração: Seja $X$ um espaço de Banach de dimensão infinita, então podemos escolher uma sequência $\left(x_{j}\right)_{j} \subset X$ tal que $\left\|x_{j}\right\|=1$ para cada $j \in \mathbb{N}$ e $\left\|x_{s}-x_{r}\right\|>\frac{1}{2}$ para cada $r \neq s$. Para $n \in \mathbb{N}$, temos que $\mathcal{P}\left({ }^{n} X\right)$ é separável, então existe $\left\{P_{k}, k \in \mathbb{N}\right\} \subset \mathcal{P}\left({ }^{n} X\right)$ enumerável denso em $\mathcal{P}\left({ }^{n} X\right)$. Assim, para cada $P \in \mathcal{P}\left({ }^{n} X\right)$ 
e cada $\epsilon>0$ existe $P_{k_{0}} \in\left\{P_{k}, k \in \mathbb{N}\right\}$ tal que $\left\|P_{k_{0}}-P\right\|<\frac{\epsilon}{3}$. Agora, o conjunto $\left\{P_{k_{0}}\left(x_{j}\right): j \in \mathbb{N}\right\}$ é limitado em $\mathbb{K}$, uma vez que

$$
\left|P_{k_{0}}\left(x_{j}\right)\right| \leq\left\|P_{k_{0}}\right\| \cdot\left\|x_{j}\right\|^{n}=\left\|P_{k_{0}}\right\|
$$

para cada $x_{j}$ (teorema 2.4.2), logo podemos escolher uma subsequência $\left(y_{j}\right)_{j}$ de $\left(x_{j}\right)_{j}$ tal que $\left(P_{k_{0}}\left(y_{j}\right)\right)_{j}$ é convergente.

Definamos as sequências $\left(a_{j}\right)_{j}$ e $\left(b_{j}\right)_{j}$ da seguinte maneira:

Para cada $j \in \mathbb{N}, a_{j}=y_{2 j}$ e $b_{j}=y_{2 j+1}$.

Logo,

$$
\left|P_{k_{0}}\left(a_{j}\right)-P_{k_{0}}\left(b_{j}\right)\right|=\left|P_{k_{0}}\left(y_{2 j}\right)-P_{k_{0}}\left(y_{2 j+1}\right)\right| \stackrel{j \rightarrow \infty}{\longrightarrow} 0 .
$$

Assim, existe $j_{0} \in \mathbb{N}$ tal que $\left|P_{k_{0}}\left(a_{j}\right)-P_{k_{0}}\left(b_{j}\right)\right|<\frac{\epsilon}{3}$ para cada $j \geq j_{0}$.

Como $\left\|P_{k_{0}}-P\right\|<\frac{\epsilon}{3}$ temos que para cada $x \in X$ com $\|x\| \leq 1,\left|P_{k_{0}}(x)-P(x)\right|<\frac{\epsilon}{3}$. Logo, para cada $j \geq j_{0}$, temos que:

$$
\left|P\left(a_{j}\right)-P\left(b_{j}\right)\right| \leq\left|P_{k_{0}}\left(a_{j}\right)-P\left(a_{j}\right)\right|+\left|P_{k_{0}}\left(b_{j}\right)-P\left(b_{j}\right)\right|+\left|P_{k_{0}}\left(a_{j}\right)-P_{k_{0}}\left(b_{j}\right)\right|<\epsilon,
$$

ou seja, $\left|P\left(a_{j}\right)-P\left(b_{j}\right)\right| \stackrel{j \rightarrow \infty}{\longrightarrow} 0$. Para cada $j \in \mathbb{N}$, temos que $\left\|a_{j}-b_{j}\right\|=\left\|y_{2 j}-y_{2 j+1}\right\|>\frac{1}{2}$ e como $X$ é polinomialmente Schur, existe $Q \in \mathcal{P}\left({ }^{n} X\right)$ tal que $\left|Q\left(a_{j}-b_{j}\right)\right| \not \rightarrow 0$. Portanto $X$ não tem a propriedade $\mathbf{P}$.

No trabalho [9], Castillo, García \& Gonzallo conseguiram exibir um exemplo de um espaço de Banach que não possui a propriedade $\mathbf{P}$. Tal espaço é dado por $X \times Y$ onde $X$ e $Y$ tem a propriedade $\mathbf{P}$. $\mathrm{O}$ exemplo trabalha com o espaço de sequências de Lorentz e seu predual (ambos definidos no capítulo 1, seção 2).

Castillo, Garcia \& Gonzallo, em [9] demonstraram que nos espaços $d_{*}(w ; 1)$ e $d(w ; 1)$, onde para cada $p>1, w=\left(w_{i}\right)_{i}$ satisfaz $(\Delta)$ :

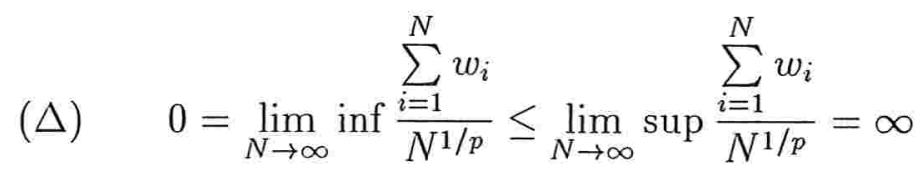

valem as seguintes afirmações:

$$
\begin{gathered}
\mathcal{P}\left(d_{*}(w ; 1)\right)=\mathcal{P}_{w s c}\left(d_{*}(w ; 1)\right) \\
\mathcal{P}(d(w ; 1))=\mathcal{P}_{w s c}(d(w ; 1)) .
\end{gathered}
$$

Tais igualdades serão utilizadas na demonstração do próximo teorema. 
Teorema 3.2.22. Seja $d(w ; 1)$ o espaço de sequências de Lorentz tal que para cada $p>1$, $w=\left(w_{i}\right)_{i}$ satisfaz $(\Delta)$. Então o espaço $d_{*}(w ; 1) \times d(w ; 1)$ não tem a propriedade $\mathbf{P}$.

Demonstração: Vamos considerar em $d_{*}(w ; 1) \times d(w ; 1)$ a norma dada pelo sup. Sejam $\left(e_{n}\right)_{n}$ a base canônica de $d(w ; 1)$ e $\left(e_{n}^{*}\right)_{n}$ a base de $d_{*}(w ; 1)$ associada a $\left(e_{n}\right)_{n}$. Então as sequências $\left(\left(e_{n}^{*}, 0\right)\right)_{n}$ e $\left(\left(0, e_{n}\right)\right)_{n}$ são limitadas em $d_{*}(w ; 1) \times d(w ; 1), e_{n}^{*} \stackrel{\omega}{\rightarrow} 0$ e $e_{n} \stackrel{\omega}{\rightarrow} 0$ (ver [[9], teorema 5.4]. Assim, para cada $Q \in \mathcal{P}\left(d_{*}(w ; 1) \times d(w ; 1)\right)$ afirmamos que

$$
\left|Q\left(\left(e_{n}^{*}, 0\right)\right)-Q\left(\left(0, e_{n}\right)\right)\right| \stackrel{n \rightarrow \infty}{\longrightarrow} 0
$$

De fato: dado $Q \in \mathcal{P}\left(d_{*}(w ; 1) \times d(w ; 1)\right)$ considere $S: d_{*}(w ; 1) \longrightarrow \mathbb{K}$ dado por $S(x)=Q(x, 0)$ para cada $x \in d_{*}(w ; 1)$. Assim $S \in \mathcal{P}\left(d_{*}(w ; 1)\right)$ e como $e_{n}^{*} \stackrel{\omega}{\rightarrow} 0$ e $\mathcal{P}\left(d_{*}(w ; 1)\right)=\mathcal{P}_{w s c}\left(d_{*}(w ; 1)\right)$ temos que $S\left(e_{n}^{*}\right) \stackrel{n \rightarrow \infty}{\longrightarrow} 0$. Logo $Q\left(\left(e_{n}^{*}, 0\right)\right) \stackrel{\omega}{\rightarrow} 0$.

Analogamente, definindo o polinômio $R: d(w ; 1) \longrightarrow \mathbb{K}$ por $R(y)=Q((0, y))$ para cada $y \in d(w ; 1)$ temos que $R\left(e_{n}\right) \stackrel{n \rightarrow \infty}{\longrightarrow} 0$, pois $e_{n} \stackrel{\omega}{\rightarrow} 0$ e $\mathcal{P}(d(w ; 1))=\mathcal{P}_{w s c}(d(w ; 1))$. Logo $Q\left(\left(0, e_{n}\right)\right) \stackrel{n \rightarrow \infty}{\longrightarrow} 0$.

Assim,

$$
\lim _{n \rightarrow 0}\left[Q\left(\left(e_{n}^{*}, 0\right)\right)-Q\left(\left(0, e_{n}\right)\right)\right]=0
$$

ou seja,

$$
\left|Q\left(\left(e_{n}^{*}, 0\right)\right)-Q\left(\left(0, e_{n}\right)\right)\right| \stackrel{n \rightarrow \infty}{\longrightarrow} 0 .
$$

No que segue, vamos construir um polinômio $P$ de modo que $\left|P\left(\left(e_{n}^{*}, 0\right)-\left(0, e_{n}\right)\right)\right| \nrightarrow 0$. Para isso, vamos tomar a aplicação

$A:\left(d_{*}(w ; 1) \times d(w ; 1)\right) \times\left(d_{*}(w ; 1) \times d(w ; 1)\right) \longrightarrow \mathbb{R}$ dada por:

$$
A((x, y),(u, v))=\langle x, v\rangle .
$$

Facilmente vemos que $A$ é 2-linear, pois é consequência imediata das propriedades do produto interno sobre o corpo $\mathbb{R}$.

Sejam $(x, y),(u, v) \in d_{*}(w ; 1) \times d(w ; 1)$ então:

$$
|A((x, y),(u, v))|=|\langle x, v\rangle| \leq\|x\| \cdot\|v\| \leq\|(x, y)\| \cdot\|(u, v)\| .
$$

Logo $A$ é contínuo.

Agora, definamos $P: d_{*}(w ; 1) \times d(w ; 1) \longrightarrow \mathbb{R}$ por:

$$
P((x, y))=A((x, y),(x, y))
$$

então $P$ é um polinômio 2-homogêneo e contínuo.

Assim, para cada $n \in \mathbb{N}$,

$$
\left|P\left(\left(e_{n}^{*}, 0\right)-\left(0, e_{n}\right)\right)\right|=\left|P\left(e_{n}^{*},-e_{n}\right)\right|=\left|\left\langle e_{n}^{*},-e_{n}\right\rangle\right|=1 .
$$

Portanto $d_{*}(w ; 1) \times d(w ; 1)$ não tem a propriedade $\mathbf{P}$. 
Como $\mathcal{P}\left(d_{*}(w ; 1)\right)=\mathcal{P}_{w s c}\left(d_{*}(w ; 1)\right)$ e $\mathcal{P}(d(w ; 1))=\mathcal{P}_{w s c}(d(w ; 1))$ então, pelo corolário 3.2.16, os espaços $d(w ; 1)$ e $d_{*}(w ; 1)$ têm a propriedade P. Em [9], Castillo, Garcia \& Gonzalo mostram que os espaços $d(w ; 1)$ e $d_{*}(w ; 1)$, onde $w=\left(w_{i}\right)_{i}$ satisfaz $(\Delta)$, não são Dunford-Pettis. Assim, temos mais dois exemplos de espaços de Banach que não satisfazem $(d) \Longrightarrow(a)$ no teorema 3.2.4.

O espaço $d_{*}(w ; 1) \times d(w ; 1)$ não é polinomialmente Schur, pois como vimos na demonstração do teorema 3.2 .22 , para a sequência $\left(\left(0, e_{n}\right)\right)_{n}, Q\left(\left(0, e_{n}\right)\right) \stackrel{n \rightarrow \infty}{\longrightarrow} 0$ para cada $Q \in \mathcal{P}\left(d_{*}(w ; 1) \times d(w ; 1)\right)$, mas para cada $n \in \mathbb{N}$ temos que:

$$
\left\|\left(0, e_{n}\right)\right\|_{\infty}=\left\|\left(0, e_{n}\right)\right\|_{p}=1,
$$

logo, pelo teorema 3.2.4 temos que o espaço $d_{*}(w ; 1) \times d(w ; 1)$ não tem a propriedade $(\star \star)$.

Sejam $\left(e_{n}\right)_{n}$ a base canônica de $d(w ; 1)$ e $\left(e_{n}^{*}\right)_{n}$ a base de $d_{*}(w ; 1)$ associada a $\left(e_{n}\right)_{n}$. Como $e_{n}^{*} \stackrel{\omega}{\rightarrow} 0$ e $e_{n} \stackrel{\omega}{\rightarrow} 0$ então $P\left(e_{n}\right) \stackrel{n \rightarrow \infty}{\rightarrow} 0$ para cada $P \in \mathcal{P}(d(w ; 1))$ e $Q\left(e_{n}^{*}\right) \stackrel{n \rightarrow \infty}{\longrightarrow} 0$ para cada $Q \in \mathcal{P}\left(d_{*}(w ; 1)\right)$. Para cada $n \in \mathbb{N},\left\|e_{n}\right\|=1$ e $\left\|e_{n}^{*}\right\|=1$. Assim, os espaços $d(w ; 1)$ e $d_{*}(w ; 1)$ não são polinomialmente Schur, logo, pelo teorema $3.2 .4, d(w ; 1)$ e $d_{*}(w ; 1)$ não satisfazem a propriedade $(\star \star)$. 


\section{Capítulo 4}

\section{Recíproca da propriedade $\mathbf{P}$}

\subsection{Propriedade RP}

Neste capítulo temos por objetivo estudar a recíproca da propriedade $\mathbf{P}$, que Aron, Choi \& Llavona em [2] denotaram por RP. Vamos estudar as suas relações com as propriedades Dunford-Pettis, polinomialmente Schur como também resultados que garantem que os espaços $L_{\infty}[0,1], l_{\infty}, L_{1}[0,1], L_{1}[0,2 \pi]$ e $C[0,1]$ não têm a propriedade $\mathbf{R P}$.

Definição 4.1.1. Seja $X$ um espaço de Banach. Dizemos que $X$ tem a propriedade RP se para quaisquer duas sequências $\left(u_{j}\right)_{j},\left(v_{j}\right)_{j}$ limitadas em $X$ tais que para cada $n \geq 1$ e cada $P \in \mathcal{P}\left({ }^{n} X\right),\left|P\left(u_{j}-v_{j}\right)\right| \stackrel{j \rightarrow \infty}{\longrightarrow} 0$ temos que $\left|Q\left(u_{j}\right)-Q\left(v_{j}\right)\right| \stackrel{j \rightarrow \infty}{\longrightarrow 0}$ para cada $Q \in \mathcal{P}\left({ }^{m} X\right)$ e $m \geq 1$.

Em outras palavras, dizemos que um espaço de Banach $X$ tem a propriedade $\mathbf{R P}$ se para cada polinômio $P \in \mathcal{P}(X)$ e cada $W \subset X$ limitado $\left.P\right|_{W}$ é uniformemente sequencialmente contínuo na topologia polinomial fraca.

No que segue vamos apresentar alguns resultados estudados. $\mathrm{O}$ seguinte teorema encontra-se demonstrado em [2].

Teorema 4.1.2. Seja $X$ um espaço de Banach. Valem as seguintes afirmações:

1. Se X é polinomialmente Schur então X tem a propriedade RP.

2. Se $X=c_{0}$, então $X$ tem a propriedade $\mathbf{R P}$. 
Demonstração: (1) Sejam $\left(u_{j}\right)_{j},\left(v_{j}\right)_{j}$ sequências limitadas em $X$ e suponhamos que

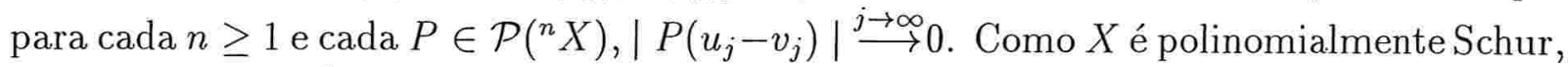
então $\left\|u_{j}-v_{j}\right\| \stackrel{j \rightarrow \infty}{\longrightarrow} 0$. Sejam $m \in \mathbb{N}$ e $Q \in \mathcal{P}\left({ }^{m} X\right)$. Como $Q$ uniformemente contínuo sobre os limitados (ver teorema 2.4.10), segue do teorema 1.2.29 que $\left|Q\left(u_{j}\right)-Q\left(v_{j}\right)\right| \stackrel{j \rightarrow \infty}{\longrightarrow} 0$. Portanto $X$ tem a propriedade RP.

(2) Sejam $\left(u_{j}\right)_{j},\left(v_{j}\right)_{j}$ sequências limitadas em $c_{0}$ e suponhamos que para cada $n \geq 1$ e cada $P \in \mathcal{P}\left({ }^{n} c_{0}\right)$ temos que $\left|P\left(u_{j}-v_{j}\right)\right| \stackrel{j \rightarrow \infty}{\longrightarrow} 0$. Consequentemente, $u_{j}-v_{j} \stackrel{\omega}{\rightarrow} 0$. Sejam $m \in \mathbb{N}$ e $Q \in \mathcal{P}\left({ }^{m} c_{0}\right)$, então pelo teorema de Littlewwood-Bogdanowicz-Pelczynski (2.5.14) existe uma sequência $\left(Q_{n}\right)_{n} \subset \mathcal{P}_{f}\left({ }^{m} c_{0}\right)$ tal que $Q_{n} \stackrel{n \rightarrow \infty}{\longrightarrow} Q$. Logo dado $\epsilon>0$ existe $n_{0} \in \mathbb{N}$ tal que $\left\|Q_{n_{0}}-Q\right\|<\frac{\epsilon}{3}$. Como $Q_{n_{0}} \in \mathcal{P}_{f}\left({ }^{m} c_{0}\right)$, segue que $Q_{n_{0}}(x)=\sum_{i=1}^{r_{n_{0}}} \lambda_{i} \psi_{i}^{m}(x)$ com $\lambda_{i} \in \mathbb{K}, \psi_{i} \in c_{0}^{*}$ para cada $i=1, \ldots, r_{n_{0}}$ e cada $x \in c_{0}$. Consequentemente, para cada $j \in \mathbb{N}$,

$$
\begin{gathered}
\left|Q_{n_{0}}\left(u_{j}\right)-Q_{n_{0}}\left(v_{j}\right)\right|=\left|\sum_{i=1}^{r_{n_{0}}} \lambda_{i}\left(\psi_{i}^{m}\left(u_{j}\right)-\psi_{i}^{m}\left(v_{j}\right)\right)\right| \\
\leq \sum_{i=1}^{r_{n_{0}}}\left|\lambda_{i}\right| \cdot\left|\psi_{i}^{m}\left(u_{j}\right)-\psi_{i}^{m}\left(v_{j}\right)\right| .
\end{gathered}
$$

De maneira análoga a proposição $3.2 .2(2)$ temos que para cada $i=1, \ldots, r_{n_{0}}$ e cada $\psi_{i} \in c_{0}^{*},\left|\psi_{i}^{m}\left(u_{j}\right)-\psi_{i}^{m}\left(v_{j}\right)\right| \stackrel{j \rightarrow \infty}{\longrightarrow} 0, \operatorname{logo},\left|Q_{n_{0}}\left(u_{j}\right)-Q_{n_{0}}\left(v_{j}\right)\right| \stackrel{j \rightarrow \infty}{\longrightarrow} 0$. Assim, existe $j_{0} \in \mathbb{N}$ tal que $\left|Q_{n_{0}}\left(u_{j}\right)-Q_{n_{0}}\left(v_{j}\right)\right|<\frac{\epsilon}{3}$ para cada $j \geq j_{0}$. Como

$$
\left|Q\left(u_{j}\right)-Q\left(v_{j}\right)\right| \leq\left|Q\left(u_{j}\right)-Q_{n_{0}}\left(u_{j}\right)\right|+\left|Q_{n_{0}}\left(u_{j}\right)-Q_{n_{0}}\left(v_{j}\right)\right|+\left|Q_{n_{0}}\left(v_{j}\right)-Q\left(v_{j}\right)\right|
$$

temos que

$$
\left|Q\left(u_{j}\right)-Q\left(v_{j}\right)\right|<\epsilon
$$

para cada $j \geq j_{0}$, uma vez que $\left|Q_{n_{0}}\left(u_{j}\right)-Q\left(u_{j}\right)\right| \leq \frac{\epsilon}{3}$ e $\left|Q_{n_{0}}\left(v_{j}\right)-Q\left(v_{j}\right)\right| \leq \frac{\epsilon}{3}$ para cada $j \geq j_{0}$. Logo $\left|Q\left(u_{j}\right)-Q\left(v_{j}\right)\right| \stackrel{j \rightarrow \infty}{\longrightarrow} 0$. Portanto, $c_{0}$ tem a propriedade RP.

A recíproca do item (1) do teorema acima não vale, pois o espaço $c_{0}$ não é polinomialmente Schur, mas tem a propriedade RP.

No que segue, vamos dar alguns exemplos de espaços de Banach que têm a propriedade RP.

Exemplos 4.1.3. 1. Para $p, 1 \leq p<\infty$, os espaços $l_{p}$ têm a propriedade RP, pois estes espaços são polinomialmente Schur. 
2. Para $q, 2 \leq q<\infty$, os espaços $L_{q}[0,1]$ têm a propriedade $\mathbf{R P}$, pois estes espaços são polinomialmente Schur.

3. Seja X um espaço de Banach.

(a) Se $X$ é de tipo não-trivial então X tem a propriedade $\mathbf{R P}$, pois ele é polinomialmente Schur.

(b) Se X é Schur então $X$ tem a propriedade RP.

No capítulo anterior vimos que espaços com a propriedade Dunford-Pettis tem a propriedade P. A seguir vamos estudar o fato de que certos espaços com a Dunford-Pettis tem a propriedade $\mathbf{R P}$.

Proposição 4.1.4. Seja $X$ um espaço de Banach tal que $X$ tem a propriedade DunfordPettis, então $X$ tem a propriedade RP se e somente se dadas quaisquer duas sequências limitadas $\left(x_{j}\right)_{j},\left(y_{j}\right)_{j}$ em $X$ tais que $x_{j}-y_{j} \stackrel{\omega}{\rightarrow} 0$ temos que $\left|P\left(x_{j}\right)-P\left(y_{j}\right)\right| \rightarrow 0$ para cada $P \in \mathcal{P}\left({ }^{n} X\right)$ e todo $n \geq 1$.

Demonstração: $(\Rightarrow)$ Sejam $\left(x_{j}\right)_{j},\left(y_{j}\right)_{j}$ sequências limitadas em $X$ tais que $x_{j}-y_{j} \stackrel{\omega}{\rightarrow} 0$. Por hipótese, $X$ tem a propriedade Dunford-Pettis então, pela proposição 3.1.7(1), temos que $\left|P\left(x_{j}-y_{j}\right)\right| \stackrel{j \rightarrow \infty}{\longrightarrow} 0$ para cada $P \in \mathcal{P}\left({ }^{n} X\right)$ e cada $n \geq 1$. E como $X$ possui a propriedade $\mathbf{R P}$, segue que $\mid P\left(x_{j}\right)-P\left(y_{j}\right) \stackrel{j \rightarrow \infty}{\longrightarrow} 0$ para cada $P \in \mathcal{P}\left({ }^{m} X\right)$ e todo $m \geq 1$. $(\Leftarrow)$ Sejam $\left(x_{j}\right)_{j},\left(y_{j}\right)_{j}$ sequências limitadas em $X$ tais que para cada $n \geq 1$ e cada $P \in \mathcal{P}\left({ }^{n} X\right)$, temos que $\mid P\left(x_{j}-y_{j}\right) \stackrel{j \rightarrow \infty}{\longrightarrow} 0$, em particular, $x_{j}-y_{j} \stackrel{\omega}{\rightarrow} 0$. Sejam $m \geq 1$ e $Q \in \mathcal{P}\left({ }^{m} X\right)$, logo por hipótese, temos que $\left|Q\left(x_{j}\right)-Q\left(y_{j}\right)\right| \stackrel{j \rightarrow \infty}{\longrightarrow} 0$. Portanto $X$ tem a propriedade RP.

Análogo a propriedade $\mathbf{P}$, a propriedade $\mathbf{R P}$ também é preservada por isomorfismos.

Teorema 4.1.5. Sejam $X$ um espaço de Banach e $Y$ um espaço isomorfo a $X$. Se $X$ tem a propriedade RP então Y também tem a propriedade RP.

Demonstração: Sejam $\left(u_{j}\right)_{j},\left(v_{j}\right)_{j}$ sequências limitadas em $Y$ tais que $\left|P\left(u_{j}-v_{j}\right)\right| \stackrel{j \rightarrow \infty}{\longrightarrow} 0$ para cada $P \in \mathcal{P}\left({ }^{n} Y\right)$ e $n \geq 1$. Como $X$ é isomorfo a $Y$ existe $T: Y \longrightarrow X$ um isomorfismo. Assim, existem sequências limitadas $\left(x_{j}\right)_{j}$ e $\left(y_{j}\right)_{j}$ em X tais que

$$
\left(T\left(u_{j}\right)\right)_{j}=\left(x_{j}\right)_{j} \quad \text { e } \quad\left(T\left(v_{j}\right)\right)_{j}=\left(y_{j}\right)_{j}
$$


Agora, para cada $m \geq 1$ e cada $Q^{*} \in \mathcal{P}\left({ }^{m} X\right)$, pelo teorema 2.4.6, temos que $Q^{*} \circ T \in \mathcal{P}\left({ }^{m} Y\right)$. Logo, por hipótese, $\left|Q^{*} \circ T\left(u_{j}-v_{j}\right)\right| \stackrel{j \rightarrow \infty}{\longrightarrow} 0$, ou seja, $\left|Q^{*}\left(x_{j}-y_{j}\right)\right| \stackrel{j \rightarrow \infty}{\longrightarrow} 0$. Como $X$ tem a propriedade $\mathbf{R P}$ então $\left|Q\left(x_{j}\right)-Q\left(y_{j}\right)\right| \stackrel{j \rightarrow \infty}{\longrightarrow 0}$ para cada $Q \in \mathcal{P}\left({ }^{n} X\right)$ e $n \geq 1$. Sejam $m \in \mathbb{N}$ e $P^{*} \in \mathcal{P}\left({ }^{m} Y\right)$. Vamos provar que $\left|P^{*}\left(u_{j}\right)-P^{*}\left(v_{j}\right)\right| \stackrel{j \rightarrow \infty}{\longrightarrow} 0$. Para isso, definamos $R: X \longrightarrow \mathbb{K}$ por $R(x)=\left(P^{*} \circ T^{-1}\right)(x)$ para cada $x \in X$. Assim, pelo teorema 2.4.6, temos que $R \in \mathcal{P}\left({ }^{m} X\right)$, e consequentemente temos que $\left|R\left(x_{j}\right)-R\left(y_{j}\right)\right| \stackrel{j \rightarrow \infty}{\longrightarrow} 0$, ou seja, $\left|\left(P^{*} \circ T^{-1}\right)\left(x_{j}\right)-\left(P^{*} \circ T^{-1}\right)\left(y_{j}\right)\right| \stackrel{j \rightarrow \infty}{\longrightarrow} 0$. Mas para cada $j \in \mathbb{N},\left|\left(P^{*} \circ T^{-1}\right)\left(x_{j}\right)-\left(P^{*} \circ T^{-1}\right)\left(y_{j}\right)\right|=\left|P^{*}\left(u_{j}\right)-P^{*}\left(v_{j}\right)\right|$.

Logo, $\left|P^{*}\left(u_{j}\right)-P^{*}\left(v_{j}\right)\right| \stackrel{j \rightarrow \infty}{\longrightarrow} 0$. Portanto $Y$ tem a propriedade RP.

\subsection{Funções polinomialmente contínuas sobre os li- mitados}

Nesta seção vamos fazer um breve estudo sobre as funções que são polinomialmente contínuas sobre os limitados e estudar resultados que garantem que o espaço original de Tsirelson $T^{*}$ (ver construção em [31]), espaços com Dunford-Pettis têm a propriedade RP.

As funções polinomialmente contínuas sobre os limitados foram estudadas por Aron, Choi \& Llavona em [2] e [18].

Abaixo definimos funções que são polinomialmente contínuas sobre os limitados e vamos estudar os seus principais resultados, os quais se encontram em [18].

Definição 4.2.1. Sejam $X, Y$ espaços de Banach. Dizemos que uma função $f: X \longrightarrow Y$ é polinomialmente contínua sobre os limitados se para cada $\epsilon>0$ e cada $W \subset X$ limitado, existem $\delta>0$ e um conjunto finito $\left\{P_{1}, \ldots, P_{n}\right\} \subset \mathcal{P}(X)$ tais que para cada $x, y \in W$ satisfazendo $\left|P_{j}(x-y)\right|<\delta$ para cada $j=1, \ldots, n$ temos que $\|f(x)-f(y)\|<\epsilon$.

Em outras palavras, uma função $f: X \longrightarrow Y$ é polinomialmente contínua sobre os limitados se a função $f$ restrita a qualquer conjunto limitado é uniformemente contínua na topologia polinomial fraca. 
Observações 4.2.2. (a) Se $f: X \longrightarrow Y$ é uma função polinomialmente contínua sobre os limitados, então dado $\epsilon>0$ e $W \subset X$ limitado existem $\delta>0$ e um conjunto finito $\left\{P_{1}, \ldots, P_{n}\right\} \subset \mathcal{P}(X)$ tais que para cada $x, y \in W$ satisfazendo $\left|P_{j}(x-y)\right|<\delta$ para cada $j=1, \ldots, n$ temos que $\|f(x)-f(y)\|<\epsilon$. Agora, para cada $j=1, \ldots, n$ existem $m_{j} \in \mathbb{N}$ e $P_{k}^{[j]} \in \mathcal{P}\left({ }^{k} X\right), k=0,1, \ldots, m_{j}$ tais que $P_{j}=P_{0}^{[j]}+P_{1}^{[j]}+\ldots+P_{m_{j}}^{[j]}$. Assim, se $x, y \in W$ são tais que para cada $j=1, \ldots, n,\left|P_{k}^{[j]}(x-y)\right|<\frac{\delta}{m_{j}+1}$ para cada $k=0,1 \ldots, m_{j}$, então temos que

$$
\left|P_{j}(x-y)\right| \leq\left|P_{0}^{[j]}(x-y)\right|+\ldots+\left|P_{m_{j}}^{[j]}(x-y)\right|<\delta
$$

para $\operatorname{cada} j=1, \ldots, n$.

(b) Se para cada $i=1, \ldots, n, P_{i}$ for 1-homogêneo então $f$ é uma função fracamente uniformemente contínua sobre os limitados. Este caso especial foi estudado por Aron \& Prolla em [5] obtendo o espaço das funções que são fracamente uniformemente contínuas sobre os limitados.

Facilmente vemos que se $f: X \longrightarrow Y$ é uma função fracamente uniformemente contínua sobre os limitados então $f$ é polinomialmente contínua sobre os limitados. Assim, se uma aplicação linear $A: X \longrightarrow Y$ é compacta então $A$ é polinomialmente contínua sobre os limitados, uma vez que uma aplicação linear $A$ é compacta se $e$ somente se A é fracamente uniformemente contínua sobre os limitados (ver teorema 1.2.31).

(c) Se para cada $m \in \mathbb{N}$ temos que cada $P \in \mathcal{P}\left({ }^{m} X\right)$ é polinomialmente contínuo sobre os limitados, então todo $Q \in \mathcal{P}(X)$ é polinomialmente contínuo sobre os limitados. De fato: Seja $Q \in \mathcal{P}(X)$, então existem $m \in \mathbb{N}$ e $P_{k} \in \mathcal{P}\left({ }^{k} X\right), k=0,1, \ldots, m$ tais que $Q=P_{0}+P_{1}+\ldots+P_{m}$. Sejam $\epsilon>0$ e $W \subset X$ limitado. Para cada $k=1, \ldots, m, P_{k}$ é polinomialmente contínuo sobre os limitados, logo existem $\delta_{k}>0$ e um conjunto finito $\left\{P_{k}^{1}, \ldots, P_{k}^{n_{k}}\right\}$ de polinômios contínuos tais que se $x, y \in W$ e $\left|P_{k}^{j}(x-y)\right|<\delta_{k}$ para cada $j=1, \ldots, n_{k}$ temos que $\left|P_{k}(x)-P_{k}(y)\right|<\frac{\epsilon}{m}$. Tomando $\delta=\min \left\{\delta_{1}, \ldots, \delta_{m}\right\}$ e $F=\left\{P_{k j}: P_{k j}=P_{k}^{j}\right\}$ o conjunto formado pelos polinômios contínuos encontrados acima, se $x, y \in W$ são tais que $\left|P_{k j}(x-y)\right|<\delta$ para cada $P_{k j} \in F$ e como

$$
|Q(x)-Q(y)| \leq\left|P_{0}(x)-P_{0}(y)\right|+\left|P_{1}(x)-P_{1}(y)\right|+\ldots+\left|P_{m}(x)-P_{m}(y)\right|
$$

então $|Q(x)-Q(y)|<\epsilon$. Portanto $Q$ é polinomialmente contínuos sobre os limitados.

(d) Se uma função $f: X \longrightarrow Y$, com $X, Y$ espaços de Banach, é polinomialmente contínua sobre os limitados então temos que $f$ é uniformemente contínua sobre os limitados. De fato: Sejam $\epsilon>0$ e $W \subset X$ limitado, então existem $\delta>0$ e 
$\left\{P_{1}, \ldots, P_{n}\right\} \subset \mathcal{P}(X)$ tais que para cada $x, y \in W$ satisfazendo $\left|P_{j}(x-y)\right|<\delta$ para cada $j=1, \ldots, n$ segue que $\|f(x)-f(y)\|<\epsilon$. Agora, para cada $j=1, \ldots, n$, existem $m_{j} \in \mathbb{N}$ e $P_{k}^{[j]} \in \mathcal{P}\left({ }^{k} X\right), k=0,1, \ldots, m_{j}$ tais que $P_{j}=P_{0}^{[j]}+P_{1}^{[j]}+\ldots+P_{m_{j}}^{[j]}$. Assim, se $x, y \in W$ são tais que $\|x-y\|<\delta^{*}$, com $\delta^{*}=\min \left\{\left(\frac{\delta}{\left(m_{j}+1\right)\left\|P_{k}^{[j]}\right\|}\right)^{\frac{1}{k}} j=1, \ldots, n, \quad k=0,1, \ldots, m_{j}\right\}$, então para cada $j=1, \ldots, n$ e cada $k=0,1, \ldots, m_{j}$

$$
\left\|P_{k}^{[j]}(x-y)\right\| \leq\left\|P_{k}^{[j]}\right\|\|x-y\|^{k}<\frac{\delta}{m_{j}+1},
$$

e por (a) segue que $\left|P_{j}(x-y)\right|<\delta$ para cada $j=1, \ldots, n$ e por hipótese, $\|f(x)-f(y)\|<\epsilon$.

No que segue, vamos mostrar que no caso de um polinômio $P$, para verificar a continuidade polinomial sobre os limitados basta verificar a sua continuidade polinomial na bola unitária fechada.

Proposição 4.2.3. Sejam $X, Y$ espaços de Banach, $m \in \mathbb{N}$ e $P \in \mathcal{P}\left({ }^{m} X ; Y\right)$. Então $P$ é polinomialmente contínuo sobre os limitados se e somente se $P$ é polinomialmente continuo sobre $B_{X}$.

Demonstração: $(\Rightarrow)$ Trivial.

$(\Leftarrow)$ Seja $W \subset X$ limitado. Se $W \subset B_{X}$ segue o resultado. Caso contrário, existe $r>1$ tal que $W \subset B[0, r]$. Seja $\epsilon>0$, então existem $\delta>0$ e $\left\{P_{1}, \ldots, P_{n}\right\} \subset \mathcal{P}(X)$ tais que $\|P(x)-P(y)\|<\frac{\epsilon}{r^{m}}$ sempre que $\left|P_{j}(x-y)\right|<\delta, j=1, \ldots, n$, com $x, y \in B_{X}$. Agora, para cada $j=1, \ldots, n$ existem $m_{j} \in \mathbb{N}$ e $P_{k}^{[j]} \in \mathcal{P}\left({ }^{k} X\right), k=0,1, \ldots, m_{j}$ tais que $P_{j}=P_{0}^{[j]}+P_{1}^{[j]}+\ldots+P_{m_{j}}^{[j]}$. Seja $\delta^{*}=\min \left\{\frac{\delta}{m_{j}+1} \quad 1 \leq j \leq n\right\}$ e sejam $z, w \in W$ tais que para cada $j=1, \ldots, n,\left|P_{k}^{[j]}(z-w)\right|<\delta^{*}$ para cada $k=0,1, \ldots, m_{j}$. Assim, para cada $j=1, \ldots, n,\left|P_{k}^{[j]}(z-w)\right|<\frac{\delta}{m_{j}+1}$ para cada $k=0,1, \ldots, m_{j}$. Mas para cada $j=1, \ldots, n$ e cada $k=0,1, \ldots, m_{j}$ temos que

$$
\left|P_{k}^{[j]}\left(\frac{z}{r}-\frac{w}{r}\right)\right|=\left|P_{k}^{[j]}\left(\frac{1}{r}(z-w)\right)\right|<\left|P_{k}^{[j]}(z-w)\right| .
$$

Assim, $\left|P_{k}^{[j]}\left(\frac{z}{r}-\frac{w}{r}\right)\right|<\frac{\delta}{m_{j}+1}$ para cada $j=1, \ldots, n$ e cada $k=0,1, \ldots, m_{j}$, e, pela observação $4.2 .2(\mathrm{a})$ temos que $\left|P_{j}\left(\frac{x}{r}-\frac{y}{r}\right)\right|<\delta$ para cada $j=1, \ldots, n$, e por hipótese, $\left\|P\left(\frac{z}{r}\right)-P\left(\frac{w}{r}\right)\right\|<\frac{\epsilon}{r^{m}}$. Mas

$$
\left\|P\left(\frac{z}{r}\right)-P\left(\frac{w}{r}\right)\right\|=\frac{1}{r^{m}}\|P(z)-P(w)\|,
$$


logo, $\|P(z)-P(w)\|<r^{m} \cdot \frac{\epsilon}{r^{m}}=\epsilon$. Portanto, $P$ é polinomialmente contínuo sobre os limitados.

A demonstração do próximo resultado encontra-se em [[18], teorema 3.1.11], o qual nos dá uma relação de um polinômio polinomialmente contínuo sobre os limitados e a sua aplicação linear associada.

Proposição 4.2.4. Sejam $X, Y$ espaços de Banach e $m \in \mathbb{N}$. Um polinômio $P \in \mathcal{P}\left({ }^{m} X ; Y\right)$ é polinomialmente contínuo sobre os limitados se e somente se a aplicação linear associada ao polinômio $P, T_{P}: X \longrightarrow \mathcal{L}\left({ }^{m-1} X ; Y\right)$ definida na proposição 2.5.7, é polinomialmente contínua sobre os limitados.

Demonstração: Aqui nesta demonstração vamos denotar por $\hat{P}$ a aplicação $\hat{P} \in \mathcal{L}_{s}\left({ }^{m} X ; Y\right)$ tal que $\hat{P}(x, \stackrel{(m)}{\cdot}, x)=P(x)$ para cada $x \in X$, e no espaço $X^{m-1}$ vamos considerar a norma dada pelo sup. Pela proposição anterior, basta verificar a continuidade polinomial na bola unitária fechada $B_{X}$.

$(\Rightarrow)$ Suponhamos que $P \in \mathcal{P}\left({ }^{m} X ; Y\right)$ é polinomialmente contínuo sobre $B_{X}$ e seja $\epsilon>0$, então existem $\delta>0$ e $\left\{P_{1}, \ldots, P_{n}\right\} \subset \mathcal{P}(X)$ tais que $\|P(x)-P(y)\|<m$ ! $\epsilon$ sempre que $\left|P_{j}(x-y)\right|<\delta$ para $j=1, \ldots, n$ e $x, y \in B_{X}$. Agora, para cada $j=1, \ldots, n$ existem $m_{j} \in \mathbb{N}$ e $P_{k}^{[j]} \in \mathcal{P}\left({ }^{k} X\right), k=0,1, \ldots, m_{j}$ tais que $P_{j}=P_{0}^{[j]}+P_{1}^{[j]}+\ldots+P_{m_{j}}^{[j]}$. Seja $\delta^{*}=\min \left\{\frac{\delta}{m_{j}+1} \quad 1 \leq j \leq n\right\}$ e sejam $z, w \in B_{X}$ tais que para cada $j=1, \ldots, n$, $\left|P_{k}^{[j]}(z-w)\right|<\delta^{*}$ para cada $k=0,1, \ldots, m_{j}$. Assim, para cada $j=1, \ldots, n$, $\left|P_{k}^{[j]}(z-w)\right|<\frac{\delta}{m_{j}+1}$ para cada $k=0,1, \ldots, m_{j}$ e sejam $z_{2}, \ldots, z_{m} \in B_{X}$. Assim,

$$
\left(T_{P}(x)-T_{P}(y)\right)\left(z_{2}, \ldots, z_{m}\right)=\hat{P}\left(x, z_{2}, \ldots, z_{m}\right)-\hat{P}\left(y, z_{2}, \ldots, z_{m}\right) .
$$

Pela fórmula da Polarização (ver teorema 2.2.8), temos que:

$$
\begin{gathered}
\left(T_{P}(x)-T_{P}(y)\right)\left(z_{2}, \ldots, z_{m}\right)= \\
\frac{1}{m ! 2^{m}} \sum_{\epsilon_{i}= \pm 1 ; 1 \leq i \leq m} \epsilon_{1} \cdot \ldots \cdot \epsilon_{m}\left[\hat{P}\left(\epsilon_{1} x+\epsilon_{2} z_{2}+\ldots+\epsilon_{m} z_{m}\right)^{m}-\hat{P}\left(\epsilon_{1} y+\epsilon_{2} z_{2}+\ldots+\epsilon_{m} z_{m}\right)^{m}\right] \\
=\frac{m^{m}}{m ! 2^{m}} \sum_{\epsilon_{i}= \pm 1 ; 1 \leq i \leq m} \epsilon_{1} \cdot \ldots \cdot \epsilon_{m}\left[P\left(\frac{\epsilon_{1} x+\epsilon_{2} z_{2}+\ldots+\epsilon_{m} z_{m}}{m}\right)-P\left(\frac{\epsilon_{1} y+\epsilon_{2} z_{2}+\ldots+\epsilon_{m} z_{m}}{m}\right)\right]
\end{gathered}
$$

Para cada $j=1, \ldots, n$ e cada $k=0,1, \ldots, m_{j}$ temos que

$$
\left|P_{k}^{[j]}\left(\frac{\epsilon_{1} x+\epsilon_{2} z_{2}+\ldots+\epsilon_{m} z_{m}}{m}-\frac{\epsilon_{1} y+\epsilon_{2} z_{2}+\ldots+\epsilon_{m} z_{m}}{m}\right)\right|=\left|P_{k}^{[j]}\left(\frac{\epsilon_{1}}{m}(x-y)\right)\right|
$$




$$
\leq\left|P_{k}^{[j]}(x-y)\right|<\frac{\delta}{m_{j}+1}
$$

E como $\left\|\frac{\epsilon_{1} x+\epsilon_{2} z_{2}+\ldots+\epsilon_{m} z_{m}}{m}\right\| \leq 1$ e $\left\|\frac{\epsilon_{1} y+\epsilon_{2} z_{2}+\ldots+\epsilon_{m} z_{m}}{m}\right\| \leq 1$, temos que, $\frac{\epsilon_{1} x+\epsilon_{2} z_{2}+\ldots+\epsilon_{m} z_{m}}{m}$, $\frac{\epsilon_{1} y+\epsilon_{2} z_{2}+\ldots+\epsilon_{m} z_{m}}{m} \in B_{X}$, logo, pela observação 4.2.2(a) temos que $\left|P_{j}\left[\left(\frac{\epsilon_{1} x+\epsilon_{2} z_{2}+\ldots+\epsilon_{m} z_{m}}{m}\right)-\left(\frac{\epsilon_{1} y+\epsilon_{2} z_{2}+\ldots+\epsilon_{m} z_{m}}{m}\right)\right]\right|<\delta$ para cada $j=1, \ldots, n$, e por hipótese:

$$
\left\|P\left(\frac{\epsilon_{1} x+\epsilon_{2} z_{2}+\ldots+\epsilon_{m} z_{m}}{m}\right)-P\left(\frac{\epsilon_{1} y+\epsilon_{2} z_{2}+\ldots+\epsilon_{m} z_{m}}{m}\right)\right\|<m ! \epsilon .
$$

Desta maneira,

$$
\left\|\left(T_{P}(x)-T_{P}(y)\right)\left(z_{2}, \ldots, z_{m}\right)\right\| \leq \frac{m^{m}}{m ! 2^{m}} 2^{m} m ! \epsilon=\epsilon
$$

Assim,

$$
\left\|T_{P}(x)-T_{P}(y)\right\|=\sup _{\left\|z_{i}\right\| \leq 1,1 \leq i \leq m}\left\|\left(T_{P}(x)-T_{P}(y)\right)\left(z_{2}, \ldots, z_{m}\right)\right\| \leq \epsilon .
$$

Portanto $T_{P}$ é polinomialmente contínuo sobre $B_{X}$.

$(\Leftarrow)$ Suponhamos que $T_{P}$ é polinomialmente contínua sobre $B_{X}$ e seja $\epsilon>0$, então existem $\delta>0$ e $\left\{P_{1}, \ldots, P_{n}\right\} \subset \mathcal{P}(X)$ tais que $\left\|T_{P}(x)-T_{P}(y)\right\|<\frac{\epsilon}{m}$ sempre que $\left|P_{j}(x-y)\right|<\delta$ com $x, y \in B_{X}$ para cada $j=1, \ldots, n$. Sejam $x, y \in B_{X}$ satisfazendo as condiçóes acima, então:

$$
\begin{gathered}
P(x)-P(y)=\hat{P}(x, \stackrel{(m)}{\cdot}, x)-\hat{P}(y, \stackrel{(m)}{\cdot}, y) \\
=\hat{P}(x, \stackrel{(m)}{\cdot}, x)-\hat{P}(x, y, x, \ldots, x)+\hat{P}(x, y, x, \ldots, x) \\
-\hat{P}(x, y, y, x, \ldots, x)+\hat{P}(x, y, y, x, \ldots, x) \\
+\ldots-\hat{P}(x, y, \ldots, y)+\hat{P}(x, y, \ldots, y)-\hat{P}(y, \stackrel{(m)}{\cdot}, y)
\end{gathered}
$$

$\log$,

$$
\begin{gathered}
\|P(x)-P(y)\| \leq\|\hat{P}(x, \stackrel{(m)}{*}, x)-\hat{P}(x, y, x, \ldots, x)\| \\
+\|\hat{P}(x, y, x, \ldots, x)-\hat{P}(x, y, y, x, \ldots, x)\|+\ldots+\|\hat{P}(x, y, \ldots, y)-\hat{P}(y, \stackrel{(m)}{\cdots}, y)\| \\
=\left\|\left(T_{P}(x)-T_{P}(y)\right)\left(x,{ }^{(m-1)}, x\right)\right\|+\left\|\left(T_{P}(x)-T_{P}(y)\right)(y, x, \ldots, x)\right\| \\
+\ldots+\left\|\left(T_{P}(x)-T_{P}(y)\right)\left(y,{ }^{(m-1)}, y\right)\right\| .
\end{gathered}
$$

Assim, temos que:

$$
\begin{aligned}
\|P(x)-P(y)\| & \leq\left\|T_{P}(x)-T_{P}(y)\right\|+\stackrel{(m)}{+}+\left\|T_{P}(x)-T_{P}(y)\right\| \\
& <m \cdot\left\|T_{P}(x)-T_{P}(y)\right\| \frac{\epsilon}{m}=\epsilon .
\end{aligned}
$$

Portanto $P$ é polinomialmente contínuo sobre $B_{X}$. 
Proposição 4.2.5. Seja $m \in \mathbb{N}, m \geq 1$ e vamos assumir que $\frac{1}{p}+\frac{1}{2 m}=1$. Então cada $P \in \mathcal{P}\left({ }^{2} l_{p}\right)$ é polinomialmente contínuo sobre os limitados.

Demonstração: Seja $P \in \mathcal{P}\left({ }^{2} l_{p}\right)$ e consideremos a aplicação linear associada $T_{P}$ ao polinômio $P$. A aplicação $T_{P}: l_{p} \longrightarrow l_{p}^{*}=l_{2 m}$ é tal que $P(x)=\left\langle T_{P}(x), x\right\rangle$ para cada $x=\left(x_{i}\right)_{i} \in l_{p}$. Pela proposição 4.2 .4 basta mostrar que $T_{P}$ é polinomialmente contínua sobre os limitados. Assim, dado $\epsilon>0$ e $W \subset l_{p}$ limitado consideremos $\delta=\epsilon^{2 m} \mathrm{e}$ $R \in \mathcal{P}\left({ }^{2 m} l_{p}\right)$ dado por $R(x)=\sum_{i=1}^{\infty}\left(T_{P}\left(x_{i}\right)\right)^{2 m}$ para cada $x=\left(x_{i}\right)_{i} \in l_{p}$. Para cada $x=\left(x_{i}\right)_{i}, y=\left(y_{i}\right)_{i} \in W$ tais que $|R(x-y)|<\delta$ temos que

$$
\begin{gathered}
\left\|T_{P}(x)-T_{P}(y)\right\|_{l_{2 m}}=\left(\sum_{i=1}^{\infty}\left(T_{P}\left(x_{i}\right)-T_{P}\left(y_{i}\right)\right)^{2 m}\right)^{\frac{1}{2 m}} \\
=|R(x-y)|^{\frac{1}{2 m}}<\delta^{\frac{1}{2 m}}=\epsilon
\end{gathered}
$$

ou seja, $T_{P}$ é polinomialmente contínua sobre os limitados.

De maneira análoga ao exemplo 2.5.11(3), temos que $P: l_{2} \longrightarrow \mathbb{K}$ dado por $P(x)=\sum_{i=1}^{\infty} x_{i}^{2}$ para cada $x=\left(x_{i}\right)_{i} \in l_{2}$ não é fracamente uniformemente contínua sobre os limitados. Como $P \in \mathcal{P}\left({ }^{2} l_{2}\right)$, pela proposição acima (4.2.5), $P$ é polinomialmente contínuo sobre os limitados. Assim, o espaço das funções que são polinomialmente contínuas sobre os limitados e o espaço das funções fracamente uniformemente contínuas sobre os limitados, em geral, são diferentes.

No que segue temos por objetivo estudar o fato que o espaço original de Tsirelson $T^{*}$, como também certos espaços de Banach com a propriedade Dunford-Pettis têm a propriedade RP. Para isso, vamos utilizar o seguinte teorema:

Teorema 4.2.6. Seja $X$ um espaço de Banach e suponhamos que $l_{1} \nrightarrow X$. Então para cada espaço de Banach $Y$ e cada $m \in \mathbb{N}$ temos que $\mathcal{P}_{\text {wu }}\left({ }^{m} X ; Y\right)=\mathcal{P}_{\text {wsc }}\left({ }^{m} X ; Y\right)$.

Demonstração: Ver por exemplo [4], proposição 2.12 pg 197.

Teorema 4.2.7. Seja $X$ um espaço de Banach. Se todo $P \in \mathcal{P}(X)$ é polinomialmente contínuo sobre os limitados então $X$ tem a propriedade RP.

Demonstração: Sejam $\left(u_{j}\right)_{j},\left(v_{j}\right)_{j}$ sequências limitadas em $X$ tais que $\left|P\left(u_{j}-v_{j}\right)\right| \rightarrow 0$ para cada $P \in \mathcal{P}\left({ }^{k} X\right)$ e cada $k \in \mathbb{N}$. Sejam $m \in \mathbb{N}$ e $Q \in \mathcal{P}\left({ }^{m} X\right)$, sendo $\left(u_{j}\right)_{j},\left(v_{j}\right)_{j}$ 
sequências limitadas em $\mathrm{X}$, então existe $B \subset X$ limitado tal que $\left(u_{j}\right)_{j},\left(v_{j}\right)_{j} \subset B$. Por hipótese $Q$ é polinomialmente contínuo sobre os limitados, então, dado $\epsilon>0$ existem $\delta>0$ e $\left\{P_{1}, \ldots, P_{n}\right\} \subset \mathcal{P}(X)$ tais que se $u, v \in B$ com $\left|P_{i}(u-v)\right|<\delta$, para $i=1, \ldots, n$ temos que $|Q(u)-Q(v)|<\epsilon$. Como $\left|P\left(u_{j}-v_{j}\right)\right| \rightarrow 0$ para cada $P \in \mathcal{P}\left({ }^{k} X\right)$ e cada $k \in \mathbb{N}$, em particular, $\left|P_{i}\left(u_{j}-v_{j}\right)\right| \rightarrow 0$ para cada $i=1, \ldots, n$. Logo, para o $\delta$ encontrado acima existe $j_{0} \in \mathbb{N}$ tal que $\left|P_{i}\left(u_{j}-v_{j}\right)\right|<\delta$ para todo $j \geq j_{0}$ e para cada $i=1, \ldots, n$, logo, por hipótese, temos que $\left|Q\left(u_{j}\right)-Q\left(v_{j}\right)\right|<\epsilon$ para cada $j \geq j_{0}$. Portanto $X$ tem a propriedade RP.

Corolário 4.2.8. Seja $X$ um espaço de Banach. Se X tem a propriedade Dunford-Pettis e $l_{1} \nrightarrow X$ então $X$ tem a propriedade RP.

Demonstração: Seja $X$ um espaço de Banach com a propriedade Dunford-Pettis, então, pelo teorema 3.1.7(1), para cada $m \in \mathbb{N}$ temos que $\mathcal{P}\left({ }^{m} X\right)=\mathcal{P}_{\text {wsc }}\left({ }^{m} X\right)$. Como $l_{1} \hookrightarrow X$ então pelo teorema 4.2.6, para cada $m \in \mathbb{N}$ temos que $\mathcal{P}_{\text {wsc }}\left({ }^{m} X\right)=\mathcal{P}_{w u}\left({ }^{m} X\right)$, logo, cada polinômio $P \in \mathcal{P}(X)$ é polinomialmente contínuo sobre os limitados. Portanto, pelo teorema $4.2 .7, X$ tem a propriedade $\mathbf{R P}$.

Observamos que o teorema 4.2.7 nos dá uma outra maneira de mostrar que $c_{0}$ tem a propriedade RP pois $l_{1} \nrightarrow c_{0}$ e $c_{0}$ tem a propriedade Dunford-Pettis.

Corolário 4.2.9. O espaço original de Tsirelson $T^{*}$ tem a propriedade RP.

Demonstração: Alencar, Aron \& Dineen em [1] provaram que para cada $m \in \mathbb{N}$, $\mathcal{P}\left({ }^{m} T^{*}\right)=\mathcal{P}_{w s c}\left({ }^{m} T^{*}\right)$. Como $l_{1} \hookrightarrow T^{*}$, pelo teorema 4.2 .6 , temos que cada polinômio contínuo sobre $T^{*}$ é polinomialmente contínuo sobre os limitados. Portanto, pelo teorema 4.2.7, $T^{*}$ tem a propriedade RP.

No seguinte exemplo vamos ver que a recíproca do teorema 4.2 .7 não vale.

Exemplo 4.2.10. Seja $P \in \mathcal{P}\left({ }^{2} l_{1}\right)$ dado por:

$$
P(x)=\sum\left\{x_{j} x_{k}: \quad j \text { par }, 1 \leq k<j\right\}
$$

para cada $x=\left(x_{i}\right)_{i} \in l_{1}$. O polinômio $P$ assim definido não é polinomialmente contínuo sobre os limitados.

De fato: Aron, Cole \& Gamelin em [3], pg 83, provaram que o operador associado $T_{P}$ ao polinômio $P$ não é fracamente compacto. Agora, usando o resultado provado por 
Gutiérrez \& Llavona, [[20], teorema 3], o qual diz que todo operador polinomialmente contínuo sobre os limitados é fracamente compacto, temos que $T_{P}$ não é polinomialmente contínuo sobre os limitados, logo, pela proposição 4.2 .4 temos que $P$ não é polinomialmente contínuo sobre os limitados. E o espaço $l_{1}$ tem a propriedade RP.

\subsection{Exemplos de alguns espaços que não possuem a propriedade $R P$}

Nesta seção temos por objetivo estudar alguns espaços de Banach que não tem a propriedade RP.

A seguir vamos estudar a demostração de um lema que utilizamos na demonstração de que os espaços $L_{\infty}[0,1] \approx l_{\infty}$ não possuem a propriedade RP. Tais demonstrações foram feitas por Aron, Choi \& Llavona em [2].

Lema 4.3.1. Seja $\left(I_{j}\right)_{j}$ uma sequência de intervalos disjuntos de $[0,1]$, onde para cada $j \in \mathbb{N}, I_{j}$ não é um conjunto unitário. Então $\left(\chi_{I_{j}}\right)_{j}$ converge fracamente para zero em $L_{\infty}[0,1]$, onde para cada $j \in \mathbb{N}, \chi_{I_{j}}$ é a função característica, $\chi_{I_{j}}:[0,1] \longrightarrow\{0,1\}$ dada por: $\chi_{I_{j}}(t)=\left\{\begin{array}{lll}1 & \text { se } & t \in I_{j} \\ 0 & \text { se } & t \notin I_{j}\end{array}\right.$.

Demonstração: Suponhamos, por absurdo, que $\left(\chi_{I_{j}}\right)_{j}$ não converge fracamente para zero em $L_{\infty}[0,1]$, então existe $\varphi \in\left(L_{\infty}[0,1]\right)^{*}$ tal que $\varphi\left(\chi_{I_{j}}\right) \not \rightarrow \varphi(0)=0$. Desta maneira, existem uma subsequência de $\left(\chi_{I_{j}}\right)_{j}$, que vamos denotar por $\left(\chi_{I_{j}}\right)_{j}$, e $\epsilon>0$ tais que $\left|\varphi\left(\chi_{I_{j}}\right)\right| \geq \epsilon$ para todo $j \in \mathbb{N}$.

Sem perda de generalidade, podemos supor que $\|\varphi\|=1$.

Mas para cada $j \in \mathbb{N}$,

$$
\varphi\left(\chi_{I_{j}}\right)=\left|\varphi\left(\chi_{I_{j}}\right)\right| \cdot e^{i \theta} \quad \text { para algum } \theta \in[0,2 \pi) .
$$

Tomemos $\varphi_{1}$ tal que para cada $j \in \mathbb{N}$,

$$
\varphi_{1}\left(\chi_{I_{j}}\right)=\varphi\left(\chi_{I_{j}}\right) \cdot e^{-i \theta}
$$

então $\varphi_{1} \in\left(L_{\infty}[0,1]\right)^{*}$ e $\varphi_{1}\left(\chi_{I_{j}}\right) \geq \epsilon$ para cada $j \in \mathbb{N}$ e

$$
\left\|\varphi_{1}\right\|=\sup _{j \in \mathbb{N}}\left|\varphi\left(\chi_{I_{j}}\right)\right| \cdot\left|e^{-i \cdot \theta}\right|=\sup _{j \in \mathbb{N}}\left|\varphi\left(\chi_{I_{j}}\right)\right| \cdot 1=\|\varphi\|=1 .
$$


Seja $f=\sum_{j=1}^{\infty} \chi_{I_{j}}$ então $f \in L_{\infty}[0,1]$ e $\|f\|_{\infty}=1$.

De fato:

Seja $a \in[0,1]$ então $f(a)=\left\{\begin{array}{lll}1 & \text { se } a \in I_{j} & \text { para algum } j \in \mathbb{N} \\ 0 & \text { se } a \notin I_{j} & \text { para todo } j \in \mathbb{N}\end{array}\right.$, logo $f$ é limitada, mensurável e $\|f\|_{\infty}=\sup _{t \in[0,1]}|f(t)|=1$.

Para cada $n \in \mathbb{N}$, temos:

$$
\begin{gathered}
\varphi_{1}(f)=\varphi_{1}\left(\sum_{j=1}^{n} \chi_{I_{j}}+\sum_{j=n+1}^{\infty} \chi_{I_{j}}\right)=\sum_{j=1}^{n} \varphi_{1}\left(\chi_{I_{j}}\right)+\varphi_{1}\left(\sum_{j=n+1}^{\infty} \chi_{I_{j}}\right) \\
\geq \sum_{j=1}^{n} \epsilon+\varphi_{1}\left(\sum_{j=n+1}^{\infty} \chi_{I_{j}}\right)=n \epsilon+\varphi_{1}\left(\sum_{j=n+1}^{\infty} \chi_{I_{j}}\right)=n \epsilon+\left|\varphi_{1}\left(\sum_{j=n+1}^{\infty} \chi_{I_{j}}\right)\right| \\
\geq n \epsilon-\left|\varphi_{1}\left(\sum_{j=n+1}^{\infty} \chi_{I_{j}}\right)\right| \geq n \epsilon-\left\|\varphi_{1}\right\|=n \epsilon-1 .
\end{gathered}
$$

Logo $\left|\varphi_{1}(f)\right| \rightarrow \infty$ quando $n \rightarrow \infty$, e isto contradiz o fato de $\left|\varphi_{1}(f)\right| \leq\left\|\varphi_{1}\right\|=1$.

Portanto $\left(\chi_{I_{j}}\right)_{j}$ converge fracamente a zero em $L_{\infty}[0,1]$.

Teorema 4.3.2. O espaço $L_{\infty}[0,1] \approx l_{\infty}$ não tem a propriedade RP.

Demonstração: Seja $I_{j}=\left(\frac{1}{2^{j}}, \frac{1}{2^{j-1}}\right) j=1,2, \ldots$, e seja $\left(r_{j}(t)\right)_{j}$ as funções de Rademacher sobre $[0,1]$ definidas por $r_{j}(t)=\operatorname{sign}\left(\operatorname{sen} 2^{j} \pi t\right)$ para cada $t \in[0,1]$ (para mais detalhes ver capítulo 1 , seção 2 definição 1.2.35).

Definamos $A: L_{\infty}[0,1] \times L_{\infty}[0,1] \times L_{\infty}[0,1] \longrightarrow \mathbb{K}$ por:

$$
A(f, g, h)=\sum_{j=1}^{\infty}\left(2^{j} \int_{I_{j}} f d x\right) \cdot\left\langle g, r_{j}\right\rangle \cdot\left\langle h, r_{j}\right\rangle
$$

onde $\left\langle g, r_{j}\right\rangle=\int_{0}^{1} g \cdot r_{j} d x$ para cada $g \in L_{\infty}[0,1]$.

Sejam $f, g, h \in L_{\infty}[0,1]$ e $n \in \mathbb{N}$, então:

$$
\begin{gathered}
\left|\sum_{j=1}^{n}\left(2^{j} \int_{I_{j}} f d x\right) \cdot\left\langle g, r_{j}\right\rangle \cdot\left\langle h, r_{j}\right\rangle\right| \leq \sum_{j=1}^{n}\left|2^{j} \int_{I_{j}} f d x\right| \cdot\left|\left\langle g, r_{j}\right\rangle \cdot\left\langle h, r_{j}\right\rangle\right| \\
\leq \sum_{j=1}^{n}\left(2^{j} \int_{I_{j}}|f(x)| d x\right) \cdot\left|\left\langle g, r_{j}\right\rangle \cdot\left\langle h, r_{j}\right\rangle\right|
\end{gathered}
$$




$$
\begin{aligned}
& \leq \sum_{j=1}^{n}\left(2^{j} \int_{I_{j}}\|f\|_{\infty} d x\right) \cdot\left|\left\langle g, r_{j}\right\rangle \cdot\left\langle h, r_{j}\right\rangle\right| \\
&=\|f\|_{\infty} \sum_{j=1}^{n}\left(2^{j} \int_{I_{j}} d x\right) \cdot\left|\left\langle g, r_{j}\right\rangle \cdot\left\langle h, r_{j}\right\rangle\right| \\
&=\|f\|_{\infty} \sum_{j=1}^{n}\left(2^{j} \cdot \frac{1}{2^{j}}\right) \cdot\left|\left\langle g, r_{j}\right\rangle \cdot\left\langle h, r_{j}\right\rangle\right| \\
&=\|f\|_{\infty} \sum_{j=1}^{n}\left|\left\langle g, r_{j}\right\rangle \cdot\left\langle h, r_{j}\right\rangle\right| \leq\|f\|_{\infty} \cdot\left(\sum_{j=1}^{n}\left|\left\langle g, r_{j}\right\rangle^{2}\right|\right)^{1 / 2} \cdot\left(\sum_{j=1}^{n}\left|\left\langle h, r_{j}\right\rangle^{2}\right|\right)^{1 / 2} .
\end{aligned}
$$

A última desigualdade segue da desigualdade de Hölder. Como $L_{\infty}[0,1] \subset L_{2}[0,1]$ e $\left(r_{n}\right)_{n}$ forma um sistema ortonormal em $L_{2}[0,1]$ (proposição 1.2.36) usando a desigualdade de Bessel, $\sum_{j=1}^{\infty}\left|\left\langle g, r_{j}\right\rangle\right|^{2} \leq\|g\|^{2}<\infty$ para cada $g \in L_{\infty}[0,1]$, temos que

$$
|A(f, g, h)| \leq\|f\|_{\infty} \cdot\|g\|_{2} \cdot\|h\|_{2}
$$

e $A(f, g, h) \in \mathbb{K}$, consequentemente

$$
|A(f, g, h)| \leq\|f\|_{\infty} \cdot\|g\|_{\infty} \cdot\|h\|_{\infty},
$$

uma vez que

$$
\|g\|_{2}=\left(\int_{0}^{1}|g(t)|^{2} d t\right)^{2} \leq\left(\int_{0}^{1}\|g\|_{\infty}^{2} d t\right)^{1 / 2}=\|g\|_{\infty}
$$

Agora, é fácil ver que a aplicação $A$ é 3-linear e pelo teorema 2.1.2 temos que é contínua. Para cada $j \in \mathbb{N}$, seja:

$$
s_{j}=\chi_{I_{j}} \quad, \quad x_{j}=r_{j} \quad, \quad y_{j}=r_{j}+s_{j} .
$$

Por definição, $\left(x_{j}\right)_{j}$ e $\left(y_{j}\right)_{j}$ são sequências limitadas em $L_{\infty}[0,1]$. Como $y_{j}-x_{j}=s_{j}$ para cada $j \in \mathbb{N}$, segue do lema 4.3 .1 que $y_{j}-x_{j} \stackrel{\omega}{\rightarrow} 0$. Assim para cada $P \in \mathcal{P}\left({ }^{n} L_{\infty}[0,1]\right)$ e $n \geq 1$, temos que $\left|P\left(x_{j}-y_{j}\right)\right| \stackrel{j \rightarrow \infty}{\longrightarrow 0}$ uma vez que $L_{\infty}[0,1]$ tem a propriedade DunfordPettis.

Seja $Q \in \mathcal{P}\left({ }^{3} L_{\infty}[0,1]\right)$ definido por: $Q(f)=A(f, f, f)$ para cada $f \in L_{\infty}[0,1]$.

Para cada $n \in \mathbb{N}$, temos que

$$
\int_{I_{j}} r_{n} d x=\left\{\begin{array}{lll}
\frac{1}{2^{j}} & \text { se } & j \geq n+1 \\
-\frac{1}{2^{j}} & \text { se } & j=n \\
0 & \text { se } & 1 \leq j \leq n-1
\end{array}\right.
$$


onde $r_{n}$ é a função de Rademacher. Então,

$$
Q\left(x_{n}\right)=Q\left(r_{n}\right)=A\left(r_{n}, r_{n}, r_{n}\right)=\sum_{j=1}^{\infty}\left(2^{j} \int_{I_{j}} r_{n} d x\right) \cdot\left\langle r_{n}, r_{j}\right\rangle \cdot\left\langle r_{n}, r_{j}\right\rangle
$$

Como, $\left\langle r_{n}, r_{j}\right\rangle=0$ se $j \neq n$ e $\left\langle r_{n}, r_{j}\right\rangle=1$ se $j=n$, então:

$$
\begin{gathered}
Q\left(x_{n}\right)=2^{n} \int_{I_{n}} r_{n} d x=2^{n} \cdot\left(\frac{-1}{2^{n}}\right)=-1 \\
Q\left(y_{n}\right)=A\left(y_{n}, y_{n}, y_{n}\right)=A\left(r_{n}+s_{n}, r_{n}+s_{n}, r_{n}+s_{n}\right) \\
=\sum_{j=1}^{\infty}\left(2^{j} \int_{I_{j}}\left(r_{n}+s_{n}\right) d x\right) \cdot\left\langle r_{n}+s_{n}, r_{j}\right\rangle^{2}
\end{gathered}
$$

Como

$r_{n}+s_{n}=r_{n}$ sobre $I_{j}$ quando $j<n$ ou $j>n$

$r_{n}+s_{n}=0$ sobre $I_{j}$ quando $j=n$. Assim:

$$
\begin{gathered}
Q\left(y_{n}\right)=\sum_{j=1}^{n-1} 2^{j}\left(\int_{I_{j}} r_{n} d x\right)\left\langle r_{n}+s_{n}, r_{j}\right\rangle^{2}+\sum_{j=n+1}^{\infty} 2^{j}\left(\int_{I_{j}} r_{n} d x\right)\left\langle r_{n}+s_{n}, r_{j}\right\rangle^{2} \\
=\sum_{j=n+1}^{\infty} 2^{j}\left(\frac{1}{2^{j}}\right)\left\langle r_{n}+s_{n}, r_{j}\right\rangle^{2}=\sum_{j=n+1}^{\infty}\left\langle r_{n}+s_{n}, r_{j}\right\rangle^{2} \\
=\sum_{j=n+1}^{\infty}\left(\left\langle r_{n}, r_{j}\right\rangle+\left\langle s_{n}, r_{j}\right\rangle\right)^{2}=\sum_{j=n+1}^{\infty}\left\langle s_{n}, r_{j}\right\rangle^{2}
\end{gathered}
$$

$\operatorname{Mas}\left\langle s_{n}, r_{j}\right\rangle=\int_{0}^{1} s_{n} r_{j} d x=\int_{I_{n}} r_{j} d x=\left\{\begin{array}{lll}0 & \text { se } & j \geq n+1 \\ -\frac{1}{2^{n}} & \text { se } & j=n\end{array}\right.$ logo, $Q\left(y_{n}\right)=0$. Para cada $n \in \mathbb{N},\left|Q\left(x_{n}\right)-Q\left(y_{n}\right)\right|=|-1-0|=1$. Desta maneira,

$$
\left|Q\left(x_{n}\right)-Q\left(y_{n}\right)\right| \not \supset 0 \text {. }
$$

Portanto $L_{\infty}[0,1]$ não possui a propriedade $\mathbf{R P}$.

Teorema 4.3.3. O espaço $C[0,1]$ não possui a propriedade RP.

Demonstração: Seja $I_{j}=\left(\frac{1}{2^{j}}, \frac{1}{2^{j-1}}\right), j=1,2, \ldots$ e seja $\left(r_{j}(t)\right)_{j}$ as funções de Rademacher sobre $[0,1]$ definidas por $r_{j}(t)=\operatorname{sign}\left(\operatorname{sen} 2^{j} \pi t\right)$.

Definamos $A: C[0,1] \times C[0,1] \times C[0,1] \longrightarrow \mathbb{K}$ por:

$$
A(f, g, h)=\sum_{j=1}^{\infty} f\left(\frac{1}{2^{j}}\right)\left\langle g, r_{j}\right\rangle \cdot\left\langle h, r_{j}\right\rangle .
$$


Sejam $f, g, h \in C[0,1]$ e $n \in \mathbb{N}$, então

$$
\begin{gathered}
\left|\sum_{j=1}^{n} f\left(\frac{1}{2^{j}}\right)\left\langle g, r_{j}\right\rangle\left\langle h, r_{j}\right\rangle\right| \leq \sum_{j=1}^{n}\left|f\left(\frac{1}{2^{j}}\right)\right| \cdot\left|\left\langle g, r_{j}\right\rangle\left\langle h, r_{j}\right\rangle\right| \\
\leq \sum_{j=1}^{n}\|f\|_{\infty}\left|\left\langle g, r_{j}\right\rangle\left\langle h, r_{j}\right\rangle\right| \leq\|f\|_{\infty}\left(\sum_{j=1}^{n}\left|\left\langle g, r_{j}\right\rangle\right|^{2}\right)^{1 / 2} \cdot\left(\sum_{j=1}^{n}\left|\left\langle h, r_{j}\right\rangle\right|^{2}\right)^{1 / 2}
\end{gathered}
$$

pela desigualdade de Hölder, pois $C[0,1] \subset L_{\infty}[0,1]$ e $\left(\left\langle g, r_{j}\right\rangle\right)_{j} \in l_{2}$ e $\left(\left\langle h, r_{j}\right\rangle\right)_{j} \in l_{2}$. Com argumentos análogos ao teorema 4.3.2 temos que $A(f, g, h) \in \mathbb{K}$, e vale a seguinte desigualdade:

$$
|A(f, g, h)| \leq\|f\|_{\infty} \cdot\|g\|_{2} \cdot\|h\|_{2} \leq\|f\|_{\infty} \cdot\|g\|_{\infty} \cdot\|h\|_{\infty} .
$$

Não é difícil ver que $A$ é 3-linear e segue do teorema 2.1 .2 que é contínua.

Para cada $n \geq 2$ e $t \in[0,1 / 2]$, seja:

$$
f_{n}(t)= \begin{cases}0 & : 0 \leq t \leq \frac{1}{2^{n+1}} \\ 2^{n+1}\left(t-\frac{1}{2^{n+1}}\right) & : \frac{1}{2^{n+1}} \leq t \leq \frac{1}{2^{n}} \\ -2^{n+1}\left(t-\frac{3}{2^{n+1}}\right) & : \frac{1}{2^{n}} \leq t \leq \frac{3}{2^{n+1}} \\ 0 & : \frac{3}{2^{n+1}} \leq t \leq \frac{1}{2}\end{cases}
$$

Para $\frac{1}{2}+\frac{j-1}{2^{n+1}} \leq t \leq \frac{1}{2}+\frac{j}{2^{n+1}}$, onde $j=1,2, \ldots, 2^{n}$, seja:

$$
f_{n}(t)= \begin{cases}-2^{n+1}\left(t-\left(\frac{1}{2}+\frac{j}{2^{n+1}}\right)\right) & : j \equiv 0(\bmod 4) \\ -2^{n+1}\left(t-\left(\frac{1}{2}+\frac{j-1}{2^{n+1}}\right)\right) & : j \equiv 1(\bmod 4) \\ 2^{n+1}\left(t-\left(\frac{1}{2}+\frac{j}{2^{n+1}}\right)\right) & : j \equiv 2(\bmod 4) \\ 2^{n+1}\left(t-\left(\frac{1}{2}+\frac{j-1}{2^{n+1}}\right)\right) & : j \equiv 3(\bmod 4)\end{cases}
$$

Para cada $n \geq 2$, seja

$$
g_{n}=-f_{n} \chi_{[0,1 / 2]}+f_{n} \chi[1 / 2,1] .
$$

As sequências $\left(f_{n}\right)_{n=2}^{\infty}$ e $\left(g_{n}\right)_{n=2}^{\infty}$ são limitadas em $C[0,1]$, pois para cada $n \in \mathbb{N}, n \geq 2$, $-1 \leq f_{n}(t) \leq 1 \mathrm{e}-1 \leq g_{n}(t) \leq 1$ para cada $t \in[0,1]$, e para cada $n \geq 2$ temos

$$
f_{n}-g_{n}=2 f_{n} \chi_{[0,1 / 2]} \quad \text { e } \quad\left\|f_{n}-g_{n}\right\|=\left\|2 f_{n} \chi_{[0,1 / 2]}\right\| \leq 2\left\|f_{n}\right\| \cdot\left\|\chi_{[0,1 / 2]}\right\| \leq 2,
$$


logo, a sequência $\left(f_{n}-g_{n}\right)_{n}$ é limitada em $C[0,1]$. Seja $t \in[0,1]$ : Se $t \in[1 / 2,1]$ então $\left(f_{n}-g_{n}\right)(t)=\left(2 f_{n} \chi_{[0,1 / 2]}\right)(t)=0$ para cada $n \geq 2$. Se $t \in[0,1 / 2]$ existe $n_{t} \in \mathbb{N}$ tal que $\frac{3}{2^{n}+1} \leq t, \operatorname{logo} f_{n}(t)=0$ para todo $n \geq n_{t}$. Assim, $\left(f_{n}-g_{n}\right)(t)=\left(2 f_{n} \chi_{[0,1 / 2]}\right)(t)=0$ para todo $n \geq n_{t}$. Desta maneira, $\left(f_{n}-g_{n}\right)(t) \rightarrow 0$ quando $n \rightarrow \infty$ para cada $t \in[0,1]$, logo, $f_{n}-g_{n} \stackrel{\omega}{\rightarrow} 0$ em $C[0,1]$. Como $C[0,1]$ tem a propriedade Dunford-Pettis então pelo teorema 3.1.7(1) $\left|P\left(f_{n}-g_{n}\right)\right| \stackrel{n \rightarrow \infty}{\longrightarrow} 0$ para cada $P \in \mathcal{P}\left({ }^{m} C[0,1]\right), m \geq 1$. Seja $Q \in \mathcal{P}\left({ }^{3} C[0,1]\right)$ dado por: $Q(f)=A(f, f, f)$ para cada $f \in C[0,1]$. Para cada $n \geq 2$ temos:

$$
\begin{gathered}
Q\left(f_{n}\right)-Q\left(g_{n}\right)=A\left(f_{n}, f_{n}, f_{n}\right)-A\left(g_{n}, g_{n}, g_{n}\right) \\
=\sum_{j=1}^{\infty} f_{n}\left(\frac{1}{2^{j}}\right)\left\langle f_{n}, r_{j}\right\rangle^{2}-\sum_{j=1}^{\infty} g_{n}\left(\frac{1}{2^{j}}\right)\left\langle g_{n}, r_{j}\right\rangle^{2} . \\
=f_{n}\left(\frac{1}{2^{n}}\right)\left\langle f_{n}, r_{n}\right\rangle^{2}-g_{n}\left(\frac{1}{2^{n}}\right)\left\langle g_{n}, r_{n}\right\rangle^{2} \\
=\left\langle f_{n}, r_{n}\right\rangle^{2}+\left\langle g_{n}, r_{n}\right\rangle^{2}
\end{gathered}
$$

Mas,

$$
\begin{gathered}
\left\langle f_{n}, r_{n}\right\rangle=\int_{0}^{1} f_{n} r_{n} d x=\int_{\frac{1}{2^{n+1}}}^{\frac{3}{2^{n+1}}} f_{n} r_{n} d x+\int_{\frac{1}{2}}^{\frac{1}{2}+\frac{1}{2^{n}}} f_{n} r_{n} d x+\ldots+\int_{1-\frac{1}{2^{n}}}^{1} f_{n} r_{n} d x \\
=2^{n-1} \int_{\frac{1}{2}}^{\frac{1}{2}+\frac{1}{2^{n}}} f_{n} r_{n} d x=2^{n-1} \cdot \frac{1}{2^{n+1}}=\frac{1}{2^{2}} \\
\text { e }\left\langle g_{n}, r_{n}\right\rangle=\int_{\frac{1}{2}}^{1} f_{n} r_{n} d x=\frac{1}{2^{2}}
\end{gathered}
$$

logo, para cada $n \geq 2$

$$
Q\left(f_{n}\right)-Q\left(g_{n}\right)=\left(\frac{1}{2^{2}}\right)^{2}+\left(\frac{1}{2^{2}}\right)^{2}=\frac{1}{8} .
$$

Portanto, $C[0,1]$ não tem a propriedade RP.

Teorema 4.3.4. O espaço $L_{1}[0,2 \pi]$ não possui a propriedade $\mathbf{R P}$.

Demonstração: Seja $I_{j}=\left(\frac{2 \pi}{2^{j}}, \frac{2 \pi}{2^{j-1}}\right), j=1,2, \ldots$ e seja $\left(r_{j}(t)\right)_{j}$ as funções de Rademacher sobre $[0,2 \pi]$ definidas por $r_{j}(t)=\operatorname{sign}\left(\operatorname{sen} 2^{j-1} t\right)$.

Definamos A : $L_{1}[0,2 \pi] \times L_{1}[0,2 \pi] \longrightarrow \mathbb{K}$ por:

Para cada $f, g \in L_{1}[0,2 \pi]$

$$
A(f, g)=\sum_{j=1}^{\infty}\left(\int_{I_{j}} f r_{j} d x\right)\left\langle g, r_{j}\right\rangle .
$$


Sejam $f, g \in L_{1}[0,2 \pi]$ e $n \in \mathbb{N}$, então

$$
\begin{gathered}
\left|\sum_{j=1}^{n}\left(\int_{I_{j}} f r_{j} d x\right)\left\langle g, r_{j}\right\rangle\right| \leq \sum_{j=1}^{n}\left|\int_{I_{j}} f r_{j} d x\right| \cdot\left|\left\langle g, r_{j}\right\rangle\right| \\
\leq \sum_{j=1}^{n}\left(\int_{I_{j}}\left|f r_{j}\right| d x\right) \cdot\left|\left\langle g, r_{j}\right\rangle\right| \leq \sum_{j=1}^{n}\left(\int_{I_{j}}|f| \cdot\left|r_{j}\right| d x\right) \cdot\left|\int_{0}^{1} g r_{j} d x\right| \\
\leq \sum_{j=1}^{n}\left(\int_{I_{j}}\|f\|_{1} \cdot\left\|r_{j}\right\|_{1} d x\right) \cdot \int_{0}^{1}|g| \cdot\left|r_{j}\right| d x \\
\leq \sum_{j=1}^{n}\|f\|_{1} \int_{I_{j}} d x \cdot\|g\|_{1} \int_{0}^{1} d x \\
=\|f\|_{1}\|g\|_{1} \sum_{j=1}^{\infty} \int_{I_{j}}=\|f\|_{1} \cdot\|g\|_{1}
\end{gathered}
$$

Como a desigualdade vale para todo $n \in \mathbb{N}$, então

$$
|A(f, g)| \leq\|f\|_{1}\|g\|_{1},
$$

e $A(f, g) \in \mathbb{K}$. É facil ver que $A$ é 2-linear e consequentemente, pelo teorema 2.1.2 é contínua.

Para cada $j \geq 2$, seja:

$$
\begin{aligned}
& f_{j}=-\operatorname{sen} 2^{j-1} t+2 \chi_{\left[0, \frac{2 \pi}{2 j}\right]} \cdot \operatorname{sen} 2^{j-1} t+2 \chi_{\left[2 \pi-\frac{2 \pi}{2 j}, 2 \pi\right]} \cdot \operatorname{sen} 2^{j-1} t+\frac{2^{j}}{2 \pi} \cdot \chi_{I_{j}} \\
& g_{j}=-2 \operatorname{sen} 2^{j-1} t+2 \chi_{\left[0, \frac{2 \pi}{2 j}\right]} \cdot \operatorname{sen} 2^{j-1} t+2 \chi_{\left[2 \pi-\frac{2 \pi}{2 j}, 2 \pi\right]} \cdot \operatorname{sen} 2^{j-1} t+\frac{2^{j}}{2 \pi} \cdot \chi_{I_{j}}
\end{aligned}
$$

Para cada $j \in \mathbb{N}, j \geq 2 f_{j}$ e $g_{j}$ são funções contínuas e

$$
\begin{gathered}
\int_{0}^{2 \pi}\left|f_{j}(t)\right| d t \leq \int_{0}^{2 \pi}\left|\operatorname{sen} 2^{j-1} t\right| d t+2 \int_{0}^{2 \pi}\left|\chi_{\left[0, \frac{2 \pi}{2 j}\right]} \cdot \operatorname{sen} 2^{j-1} t\right| d t \\
+2 \int_{0}^{2 \pi}\left|\chi_{\left[2 \pi-\frac{2 \pi}{2 j}, 2 \pi\right]} \cdot \operatorname{sen} 2^{j-1} t\right| d t+\int_{0}^{2 \pi}\left|\frac{2^{j}}{2 \pi} \cdot \chi_{I_{j}}\right| d t \\
=\int_{0}^{2 \pi}\left|\operatorname{sen} 2^{j-1} t\right| d t+2 \int_{0}^{\frac{2 \pi}{2 j}}\left|\operatorname{sen} 2^{j-1} t\right| d t \\
+2 \int_{2 \pi-\frac{2 \pi}{2 j}}^{2 \pi}\left|\operatorname{sen} 2^{j-1} t\right| d t+\int_{I_{j}}\left|\frac{2^{j}}{2 \pi}\right| d t
\end{gathered}
$$




$$
\begin{gathered}
=2^{j-1} \int_{0}^{\frac{2 \pi}{2^{j-1}}} \operatorname{sen} 2^{j-1} t d t+2 \int_{0}^{\frac{2 \pi}{2^{j-1}}} \operatorname{sen} 2^{j-1} t d t-2 \int_{\frac{2 \pi}{2^{j-1}}}^{\frac{2 \pi}{2^{j}}} \operatorname{sen} 2^{j-1} t d t \\
+2 \int_{2 \pi-\frac{2 \pi}{2^{j}}}^{2 \pi-\frac{2 \pi}{2^{j-1}}} \operatorname{sen} 2^{j-1} t d t-2 \int_{2 \pi-\frac{2 \pi}{2^{j-1}}}^{2 \pi} \operatorname{sen} 2^{j-1} t d t+\int_{I_{j}} \frac{2^{j}}{2 \pi} d t \\
=-\frac{2^{2}}{2^{j-1}}+1<1, \quad \text { para cada } \quad j \geq 2
\end{gathered}
$$

Analogamente

$$
\begin{gathered}
\int_{0}^{2 \pi}\left|g_{j}(t)\right| d t \leq 2 \int_{0}^{2 \pi}\left|\operatorname{sen} 2^{j-1} t\right| d t+2 \int_{0}^{2 \pi}\left|\chi_{\left[0, \frac{2 \pi}{2^{j}}\right]} \cdot \operatorname{sen} 2^{j-1} t\right| d t \\
+2 \int_{0}^{2 \pi}\left|\chi_{\left[2 \pi-\frac{2 \pi}{2^{j}}, 2 \pi\right]} \cdot \operatorname{sen} 2^{j-1} t\right| d t+\int_{0}^{2 \pi}\left|\frac{2^{j}}{2 \pi} \cdot \chi_{I_{j}}\right| d t \\
=\int_{0}^{2 \pi}\left|\operatorname{sen} 2^{j-1} \cdot t\right| d t+2 \int_{0}^{\frac{2 \pi}{2^{j}}}\left|\operatorname{sen} 2^{j-1} t\right| d t \\
+2 \int_{2 \pi-\frac{2 \pi}{2^{j}}}^{2 \pi}\left|\operatorname{sen} 2^{j-1} t\right| d t+\int_{I_{j}}\left|\frac{2^{j}}{2 \pi}\right| d t \\
=2^{j-1} \int_{0}^{\frac{2 \pi}{2^{j-1}}} \operatorname{sen} 2^{j-1} t d t+2 \int_{0}^{\frac{2 \pi}{2^{j-1}}} \operatorname{sen} 2^{j-1} t d t-2 \int_{\frac{2 \pi}{2^{j}-1}}^{\frac{2 \pi}{2^{j}}} \operatorname{sen} 2^{j-1} t d t \\
+2 \int_{2 \pi-\frac{2 \pi}{2^{j}}}^{2 \pi-\frac{2 \pi}{2^{j-1}}} \operatorname{sen}^{j-1} t d t-2 \int_{2 \pi-\frac{2 \pi}{2^{j-1}}}^{2 \pi} \operatorname{sen} 2^{j-1} t d t+\int_{I_{j}} \frac{2^{j}}{2 \pi} d t \\
=-\frac{2^{2}}{2^{j-1}}+1<1, \quad \text { para cada } \quad j \geq 2 .
\end{gathered}
$$

Assim, as sequências $\left(f_{j}\right)_{j}$ e $\left(g_{j}\right)_{j}$ são limitadas em $L_{1}[0,2 \pi]$.

Para cada $j \geq 2, f_{j}-g_{j}=\operatorname{sen} 2^{j-1} t$. Assim, a sequência $\left(\left\|f_{j}-g_{j}\right\|\right)_{j \geq 2}$ é limitada. Vamos provar que $f_{j}-g_{j} \stackrel{\omega}{\rightarrow} 0$ em $L_{1}[0,2 \pi]$. Como $\left(f_{j}-g_{j}\right)_{j}$ é limitada, pelo teorema 1.2.27, basta mostrar que $\lim _{j \rightarrow \infty} \int_{E}\left(f_{j}-g_{j}\right)(t) d t=0$ para qualquer $E \subset[0,2 \pi]$ mensurárel. Primeiro, vamos considerar $E=[a, b]$, onde $0 \leq a<b \leq 1$. Então, para cada $j \in \mathbb{N}$, $\left|\int_{a}^{b} \operatorname{sen} 2^{j-1} t d t\right|=\left|\frac{-1}{2^{j-1}} \cos 2^{j-1} t\right|_{a}^{b}|=| \frac{-1}{2^{j-1}}\left(\cos 2^{j-1} b-\cos 2^{j-1} a\right) \mid \leq \frac{1}{2^{j-1}} \cdot 2=\frac{1}{2^{j-2}}$ Mas $\frac{1}{2^{j-2}} \stackrel{j \rightarrow \infty}{\longrightarrow} 0$. Assim, $\lim _{j \rightarrow \infty} \int_{E}\left(f_{j}-g_{j}\right)(t) d t=0$ para $E=[a, b]$. Logo, se $h$ é uma função escada definida em $[0,1]$, então, temos que $\int_{0}^{1} h(t)\left(f_{j}-g_{j}\right)(t) d t \stackrel{j \rightarrow \infty}{\longrightarrow} 0$, uma vez que $h(t)=\sum_{k=1}^{n} \lambda_{k} \chi_{I_{k}}(t)$ para cada $t \in[0,1]$, onde $\lambda_{k}$ são constantes e $I_{k}$ são subintervalos 
disjuntos de $[0,1]$.

Seja $E \subset[0,1]$ mensurável. Como $\chi_{E}$ é uma função limitada e mensurável, pelo teorema 1.2 .26 , existe uma sequência $\left(h_{n}\right)_{n}$ de funções escadas tal que $\int_{0}^{1}\left|\chi_{E}(t)-h_{n}(t)\right| d t \stackrel{n \rightarrow \infty}{\longrightarrow} 0$. Então dado $\epsilon>0$ existe $n_{0} \in \mathbb{N}$ tal que $\left|\int_{0}^{1}\right| \chi_{E}(t)-h_{n_{0}}(t)|d t|<\frac{\epsilon}{2}$. Como $h_{n_{0}}$ é uma função escada, temos que $\int_{0}^{1} h_{n_{0}}(t)\left(f_{j}-g_{j}\right)(t) d t \stackrel{j \rightarrow \infty}{\longrightarrow} 0$. Logo, existe $j_{0} \in \mathbb{N}$ tal que $\left|\int_{0}^{1} h_{n_{0}}(t)\left(f_{j_{0}}-g_{j_{0}}\right)(t) d t\right|<\frac{\epsilon}{2}$ para qualquer $j \geq j_{0}$. Assim,

$$
\begin{gathered}
\left|\int_{0}^{1} \chi_{E}(t)\left(f_{j}-g_{j}\right)(t) d t\right| \leq\left|\int_{0}^{1}\left(\chi_{E}(t)-h_{n_{0}}(t)\right)\left(f_{j}-g_{j}\right)(t) d t\right|+\left|\int_{0}^{1} h_{n_{0}}(t)\left(f_{j}-g_{j}\right)(t) d t\right| \\
\leq \int_{0}^{1}\left|\chi_{E}(t)-h_{n_{0}}(t)\right| d t+\frac{\epsilon}{2}<\frac{\epsilon}{2}+\frac{\epsilon}{2}=\epsilon \text { para qualquer } j \geq j_{0} .
\end{gathered}
$$

Portanto $f_{j}-g_{j} \stackrel{\omega}{\longrightarrow} 0$.

Seja $Q \in \mathcal{P}\left({ }^{2} L_{1}[0,2 \pi]\right)$ definido por: $Q(f)=A(f, f)$ para cada $f \in L_{1}[0,2 \pi]$.

Seja $j \geq 2$, então:

$$
Q\left(f_{j}\right)=A\left(f_{j}, f_{j}\right)=\sum_{i=1}^{\infty}\left(\int_{I_{i}} f_{j} r_{i} d t\right)\left\langle f_{j}, r_{i}\right\rangle
$$

Mas,

$$
\begin{gathered}
\int_{I_{i}} f_{j} r_{i} d t=-\int_{I_{i}} \operatorname{sen} 2^{j-1} t \cdot r_{i} d t+2 \int_{I_{i}} \chi_{\left[0, \frac{2 \pi}{2 j}\right]} \operatorname{sen} 2^{j-1} t \cdot r_{i} d t \\
+2 \int_{I_{i}} \chi_{\left[2 \pi-\frac{2 \pi}{2^{j}}, 2 \pi\right]} \operatorname{sen} 2^{j-1} t \cdot r_{i} d t+\int_{I_{i}} \frac{2^{j}}{2 \pi} \chi_{I_{i}} r_{i} d t=0 \quad \text { quando } \quad 1 \leq i<j \\
\left\langle f_{j}, r_{i}\right\rangle=\int_{0}^{1} f_{j} r_{i} d t=-\int_{0}^{1} \operatorname{sen} 2^{j-1} t \cdot r_{i} d t+2 \int_{0}^{1} \chi_{\left[0, \frac{2 \pi}{2^{j}}\right]} \operatorname{sen} 2^{j-1} t \cdot r_{i} d t \\
+2 \int_{0}^{1} \chi_{\left[2 \pi-\frac{2 \pi}{2^{j}}, 2 \pi\right]} \operatorname{sen} 2^{j-1} t \cdot r_{i} d t+\int_{0}^{1} \frac{2^{j}}{2 \pi} \chi_{I_{i}} r_{i} d t=0 \quad \text { quando } \quad i>j .
\end{gathered}
$$

Assim,

$$
\begin{gathered}
Q\left(f_{j}\right)=\left(\int_{I_{j}} f_{j} r_{j} d t\right)\left\langle f_{j}, r_{j}\right\rangle \\
\left\langle f_{j}, r_{j}\right\rangle=\int_{0}^{1} f_{j} r_{j} d t=-\int_{0}^{1} \operatorname{sen} 2^{j-1} t \cdot r_{j} d t+2 \int_{0}^{1} \chi_{\left[0, \frac{2 \pi}{2 j}\right]} \operatorname{sen} 2^{j-1} t \cdot r_{j} d t \\
+2 \int_{0}^{1} \chi_{\left[2 \pi-\frac{2 \pi}{2 j}, 2 \pi\right]} \operatorname{sen} 2^{j-1} t \cdot r_{j} d t+\int_{0}^{1} \frac{2^{j}}{2 \pi} \chi_{I_{i}} r_{j} d t \\
=-2^{j}\left(\frac{-1}{2^{j-2}}\right)+2\left(\frac{-1}{2^{j-2}}\right)+2\left(\frac{-1}{2^{j-2}}\right)-1=2^{2}-\left(\frac{-1}{2^{j-4}}\right)-1 \\
\int_{I_{j}} f_{j} r_{j} d t=-\int_{I_{j}} \operatorname{sen} 2^{j-1} t \cdot r_{j} d t-1=\int_{I_{j}} \operatorname{sen} 2^{j-1} t d t-1=-\frac{1}{2^{j-2}}-1 .
\end{gathered}
$$




$$
\begin{gathered}
Q\left(f_{j}\right)=-\frac{1}{2^{j-4}}+\frac{1}{2^{2 j-6}}+\frac{1}{2^{j-2}}-2^{2}+\frac{1}{2^{j-4}}+1 \\
=\frac{1}{2^{2 j-6}}+\frac{1}{2^{j-2}}-3
\end{gathered}
$$

Analogamente,

$$
\begin{gathered}
Q\left(g_{j}\right)=\left(\int_{I_{j}} g_{j} r_{j} d t\right)\left\langle g_{j}, r_{j}\right\rangle . \\
\left\langle g_{j}, r_{j}\right\rangle=2^{3}-\frac{1}{2^{j-4}}-1 \\
\int_{I_{j}} g_{j} r_{j} d t=-\frac{1}{2^{j-3}}-1 . \\
Q\left(g_{j}\right)=-\frac{1}{2^{j-6}}+\frac{1}{2^{2 j-7}}+\frac{1}{2^{j-3}}-2^{3}+\frac{1}{2^{j-4}}+1 \\
=-\frac{1}{2^{j-6}}+\frac{1}{2^{2 j-7}}+\frac{1}{2^{j-3}}+\frac{1}{2^{j-4}}-7 .
\end{gathered}
$$

Assim,

$$
Q\left(f_{j}\right)-Q\left(g_{j}\right)=\frac{1}{2^{2 j-6}}+\frac{1}{2^{j-2}}-3+\frac{1}{2^{j-6}}-\frac{1}{2^{2 j-7}}-\frac{1}{2^{j-3}}-\frac{1}{2^{j-4}}+7 \longrightarrow 4 .
$$

Portanto $L_{1}[0,2 \pi]$ não tem a propriedade $\mathbf{R P}$.

Observamos que o polinômio $Q$ acima definido não é polinomialmente contínuo sobre $B_{X}$ quando $X=L_{1}[0,2 \pi]$, pois as sequências $\left(f_{j}\right)_{j=2}^{\infty},\left(g_{j}\right)_{j=2}^{\infty} \subset B_{X}$, e $L_{1}[0,2 \pi]$ é DunfordPettis então $\left|P\left(f_{j}-g_{j}\right)\right| \stackrel{j \rightarrow \infty}{\longrightarrow} 0$ para cada $P \in \mathcal{P}\left(L_{1}[0,2 \pi]\right)$. Assim, dado $\delta>0$, para cada família finita $\left\{P_{1}, \ldots P_{n}\right\} \subset \mathcal{P}\left(L_{1}[0,2 \pi]\right)$ existe $j_{0} \in \mathbb{N}$ tal que $\left|P_{k}\left(f_{j}-g_{j}\right)\right|<\delta$ para todo $j \geq j_{0}$ e cada $k=1, \ldots, n$. Para $0<\epsilon \leq\left|Q\left(f_{j_{0}}\right)-Q\left(g_{j_{0}}\right)-4\right|$ temos que $\left|Q\left(f_{j_{0}}\right)-Q\left(g_{j_{0}}\right)\right| \geq 4 \geq \epsilon$. Portanto, o polinômio $Q$ não é polinomialmente contínuo sobre os limitados.

Analogamente vemos que os polinômios construidos nos teoremas 4.3 .2 e 4.3 .3 também não são polinomialmente contínuos sobre os limitados.

De maneira análoga ao teorema 4.3.4, também podemos provar que o espaço $L_{1}[0,1]$ não tem a propriedade RP. Basta tomar os intervalos $I_{j}=\left(\frac{1}{2^{j}}, \frac{1}{2^{j-1}}\right), j=1,2, \ldots$, $\left(r_{j}(t)\right)_{j}$ a sequência de funções de Rademacher sobre $[0,1]$ definidas por $r_{j}(t)=\operatorname{sign}\left(\operatorname{sen} 2^{j} \pi t\right)$ e a aplicação $\mathrm{A}: L_{1}[0,1] \times L_{1}[0,1] \longrightarrow \mathbb{K}$ dada por:

Para cada $f, g \in L_{1}[0,1]$

$$
A(f, g)=\sum_{j=1}^{\infty}\left(\int_{I_{j}} f r_{j} d x\right)\left\langle g, r_{j}\right\rangle
$$


Para cada $j \geq 2$, seja:

$$
\begin{aligned}
& f_{j}=-\operatorname{sen} 2^{j} \pi t+2 \chi_{\left[0, \frac{1}{2^{j}}\right]} \cdot \operatorname{sen} 2^{j} \pi t+2 \chi_{\left[1-\frac{1}{2^{j}}, 1\right]} \cdot \operatorname{sen} 2^{j} \pi t+2^{j} \cdot \chi_{I_{j}} \\
& g_{j}=-2 \operatorname{sen} 2^{j} \pi t+2 \chi_{\left[0, \frac{1}{2^{j}}\right]} \cdot \operatorname{sen} 2^{j} \pi t+2 \chi_{\left[1-\frac{1}{2^{j}}, 1\right]} \cdot \operatorname{sen} 2^{j} \pi t+2^{j} \cdot \chi_{I_{j}}
\end{aligned}
$$

e $Q \in \mathcal{P}\left({ }^{2} L_{1}[0,1]\right)$ um polinômio dado por: $Q(f)=A(f, f)$ para cada $f \in L_{1}[0,1]$.

O polinômio $Q \in \mathcal{P}\left({ }^{2} L_{1}[0,1]\right)$ acima definido também não é polinomialmente contínuo sobre os limitados. Vê-se tal fato de maneira análoga ao caso do espaço $L_{1}[0,2 \pi]$. 


\section{Referências Bibliográficas}

[1] Alencar, R., Aron, R.M. \& Dineen, S., A reflexive of holomorphic functions in infinitely many variables, Proc. Amer. Math. Soc., 90 (3) (1984) 407-411.

[2] Aron, R.M., Choi, Y. S. \& Llavona, J.G., Estimates by polynomials, Bull. Austral. Math. Soc., 52 (1995), 475-486.

[3] Aron, R.M., Cole, B.J. \& Gamelin, T.W., Spectra of algebras of analytic functions on Banach spaces, J. Reine Angew. Math., 415, (1991), 51-93.

[4] Aron, R.M., Hervés, C. \& Valdivia, M., Weakly continuous mappings on Banach spaces, J. Funct. Anal., 52 (1983) 189-204.

[5] Aron, R.M.,\& Prolla, J.B., Polynomial approximation of differentiable functions on Banach spaces, J. Reine Angew. Math., 313 (1980) 195-216.

[6] Barra, G., Introduction to Mesure Theory, Van Nostrand Reinhold Company, New York, 1974.

[7] Beauzamy, B., Introduction to Banach Spaces and their Geometry, Math. Stud. Monogr. Ser., 68, Norh-Holland, Amsterdam, 1985.

[8] Biström, P., Jaramillo, J. A. \& Lindström, M., Polynomial compactness in Banach spaces, Rocky Mountain J. Math., 28 (4) (1998) 1203-1225.

[9] Castillo, J.M.F., Garcia, R. \& Gonzalo, R., Banach spaces in which all multilinear forms are weakly sequentially continuous, Studia Math., 136 (2) (1999), 121-145.

[10] Castillo, J.M.F., \& Sanchez, C., Weakly-p-compact, p-Banach-Saks and superreflexive Banach spaces, J.Math.Anal. Appl., 185 (1994) 256-261.

[11] Chae, S.B., Holomorphy and Calculus in Normed Spaces, March Dikker, 1985.

[12] Choi, Y.S. \& Kim, S.G., Polynomial properties of Banach spaces, J. Math. Anal. Appl., 190 (1995) 203-210. 
[13] Diestel, J., Sequences and Series in Banach Spaces, Springer, 92 (1984).

[14] Diestel, J., A survey of results the Dunford-Pettis property, Proc. Conference in Integration, Topology and Geometry in Linear Spaces, W. Graves (ed) Contemp. Math. vol2, Amer. Math. Soc., Providence R.I. 1980, 15-60.

[15] Diestel, J., Jarchow, H. \& Tonge, A., Absolutely Summing Operators, Cambrige University Press, 43 (1995).

[16] Farmer, J. \& Johnson, W.B., Polynomial Schur and polynomial Dunford-Pettis properties, Contem. Math. 144 (1993) 95-105.

[17] Gamelin, T.W., Analytic functions on Banach spaces, Complex potencial theory (Montreal, PQ, 1993) 187-233, NATO Adv. Sci. Inst. Ser. C. Math. Phys. Sci., 439, Kluwer Acad. Publ., Dordrechet, 1994.

[18] Gonzalez, M., Gutiérrez, J.M. \& Llavona J.G., Polinomial continuity on $l_{1}$, Proc. Amer. Math. Soc., 125 (1997) 1349-1353.

[19] Gonzalo, R., \& Jaramillo, J. A., Compact polynomials between Banach spaces, Proc. Roy. Irish Acad. Sect.A, vol 95A , 213-226 (1995).

[20] Gutiérrez, J.M. \& Llavona, J.G., Polynomially continuos operators, Israel J. Math., 102 (1997) 179-187.

[21] Hissadomi, A.J., Propriedade de Dunford-Pettis Polinomial e Espaços Polinomialmente de Schur, Dissertação de mestrado, IME-USP (1998).

[22] James, R.C., Super-reflexive Banach spaces, Canad. J. Math., vol. XXIV, (5), (1972) 896-904.

[23] Jaramillo, J.A., \& Prieto, A., Weak-polynomial convergence on a Banach space, Proc. Amer. Math. Soc. 118 (2), (1993) 463-468.

[24] Kreyszig, E. Introductory Funtional Analysis with Applications, New York, 1978.

[25] Lindenstrauss, J. \& Tsafriri, L., Classical Banach Spaces, vol1, Springer-Verlag, Ber$\operatorname{lin},(1977)$.

[26] Littlewood, J.E., On bilinear forms in an infinite number of variables, Q. J. Math., 1 (1930), 164-174.

[27] Mujica, J., Complex Analysis in Banach Spaces, Math. Studies 120, North-Holland, Amsterdan, (1986).

[28] Rodrigues, L.P., Um Teorema de Hahn-Banach para Polinômios Homogêneos, Dissertação de mestrado, IME-USP (2001). 
[29] Ryan, R. A., Dunford-Pettis properties, Bull. Acad. Polon. Ser. Sci. Math. 27 (1979) 373-379.

[30] Sargent, W.L.C., Some sequence space related to the $l_{p}$ spaces, J. London Math. Soc. 35 (1960) 161-171.

[31] Tsirelson, B.S. Not every Banach space contains an imbedding of $l_{p}$ or $c_{0}$, Funct. Anal. Appl., 8 (1974), 138-141. 